\title{
The story of NCN as a key species in prompt-NO formation
}

\author{
Nathalie Lamoureux ${ }^{1, *}$, Pascale Desgroux ${ }^{1}$, Matthias Olzmann ${ }^{2}$, Gernot Friedrichs ${ }^{3}$ \\ ${ }^{1}$ Univ. Lille, CNRS, UMR 8522 - PC2A - Physicochimie des Processus de Combustion et de \\ l'Atmosphère, F-59000 Lille, France \\ ${ }^{2}$ Institut für Physikalische Chemie, Karlsruher Institut für Technologie (KIT), Kaiserstr. 12, 76131 \\ Karlsruhe, Germany \\ ${ }^{3}$ Institut für Physikalische Chemie, Christian-Albrechts-Universität Kiel, Max-Eyth-Straße 1, 24118 \\ Kiel, Germany
}

\section{* Corresponding author:} nathalie.lamoureux@,univ-lille.fr

Université de Lille, Lab. PC2A, Cité Scientifique, bat. C11, Av. Mendeleiev, 59655 Villeneuve d'Ascq, FRance

\begin{abstract}
:
The cyanonitrene radical, NCN, has been shown in the last two decades to play a crucial role in the formation of prompt-NO in combustion. This has stimulated a large number of experimental and theoretical studies on fundamental physico-chemical properties of NCN as well as on mechanistic and kinetic aspects of NCN reactions under combustion conditions. In this review, spectroscopic, thermodynamic, and kinetic data of NCN are collected and discussed. Methodic approaches for the detection of NCN in flames and in kinetic experiments are elucidated, and the suitability of cyanogen azide, $\mathrm{NCN}_{3}$, as a precursor for $\mathrm{NCN}$ in kinetic experiments is examined. Kinetic and mechanistic aspects of a number of NCN elementary chemical steps are extensively reviewed. Regarding prompt$\mathrm{NO}$ formation, the role of the reaction network initialized by the reaction $\mathrm{CH}+\mathrm{N}_{2} \rightleftharpoons \mathrm{NCN}+\mathrm{H}$ is examined by modeling measured flame profiles of NCN, HCN, and NO. In these simulations, the critical role of the product channel-branching of the $\mathrm{NCN}+\mathrm{H}$ reaction, termed the prompt-NO switch, is confirmed. A particularly sensitive balance is observed between the product channels leading back to $\mathrm{CH}+\mathrm{N}_{2}$ or forward to $\mathrm{HCN}+\mathrm{N}$. The roles of spin conservation and intersystem crossing processes under flame conditions and in kinetic experiments with $\mathrm{NCN}_{3}$ as $\mathrm{NCN}$ precursor are highlighted. A number of critical points and remaining open problems in NCN chemistry and prompt-NO formation are indicated.
\end{abstract}

Keywords: Cyanonitrene radical, Prompt-NO formation, Prompt-NO switch, Laser-induced fluorescence, Absorption spectroscopy, Thermochemistry, Reaction kinetics, Flame modeling 


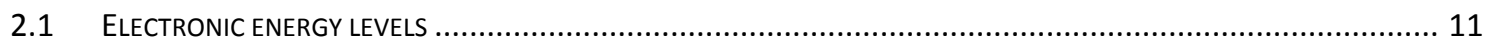

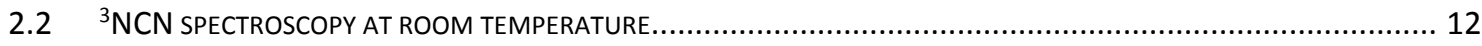

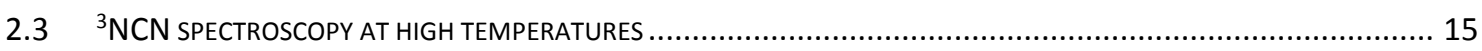

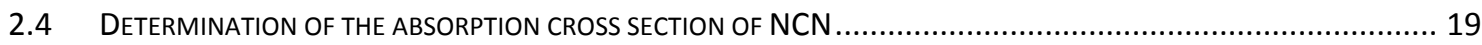

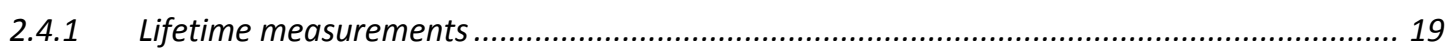

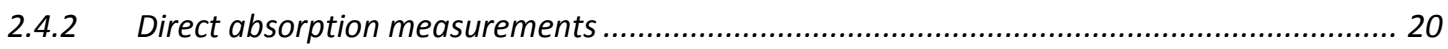

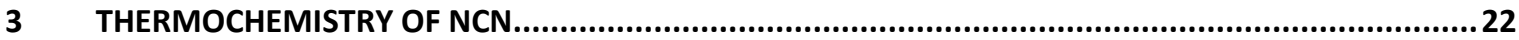

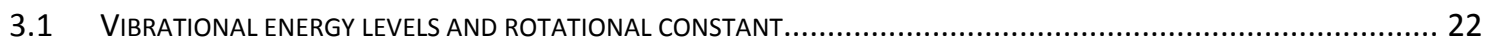

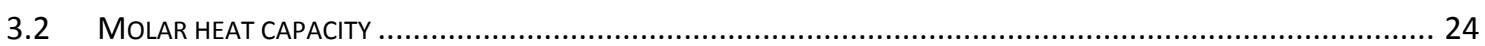

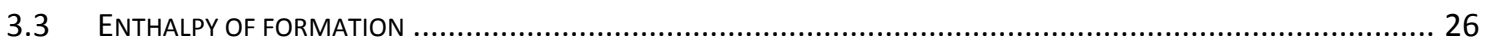

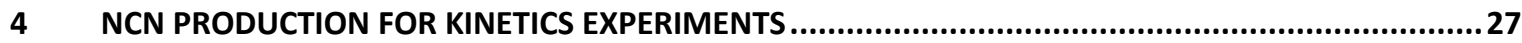

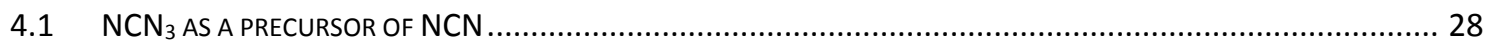

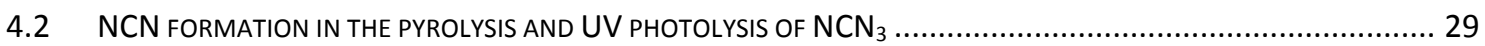

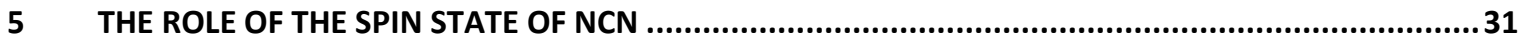

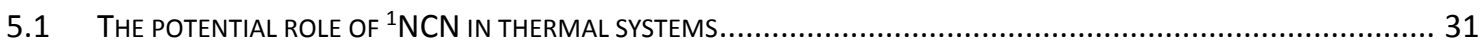

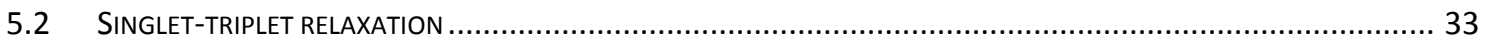

6 RATE CONSTANTS OF NCN REACTIONS FROM THEORY AND EXPERIMENT ...................................35

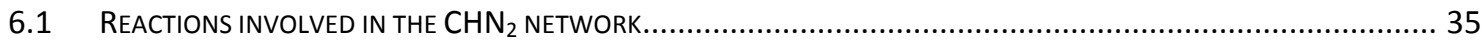

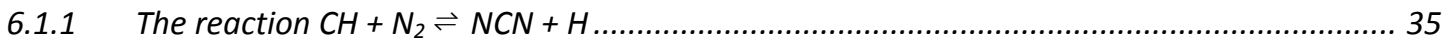

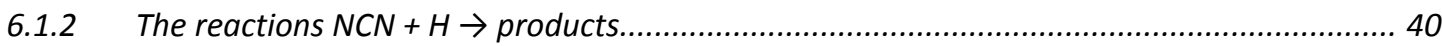

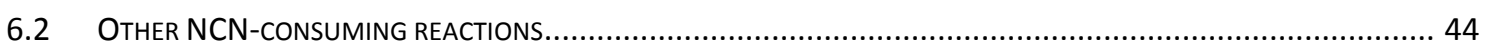

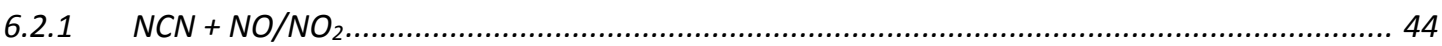

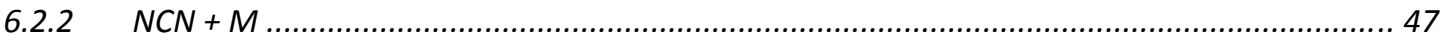

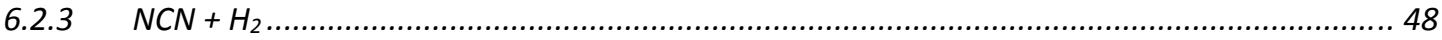

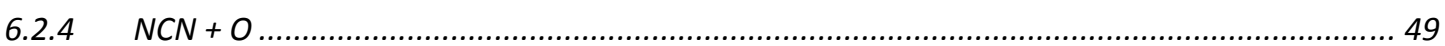

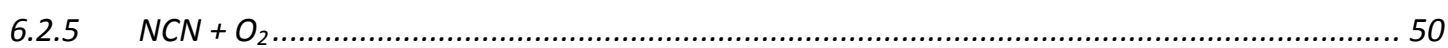

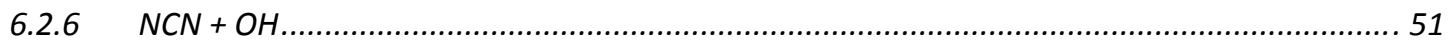

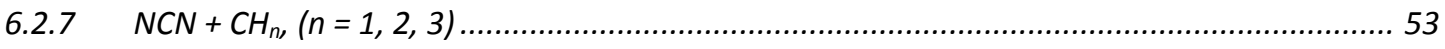

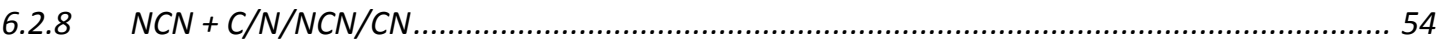

7 NCN AS A PRODUCT OF THE CH + N2 REACTION IN FLAMES: MEASUREMENT AND MODELING......55

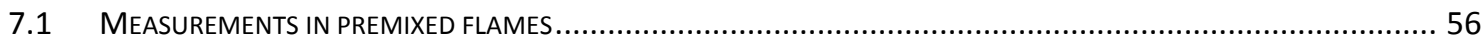

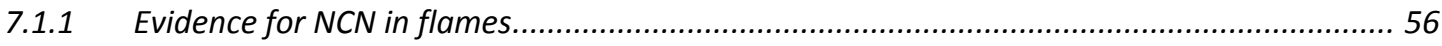


7.1.2 Absolute quantification of NCN LIF signals

7.2 IMPLEMENTATION OF THE REACTION $\mathrm{CH}+\mathrm{N}_{2} \rightleftharpoons \mathrm{NCN}+\mathrm{H}$ IN NITROGEN CHEMISTRY MODELING........................5 59

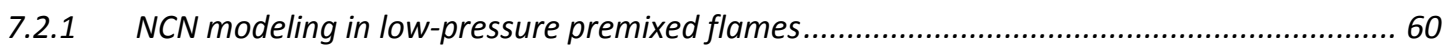

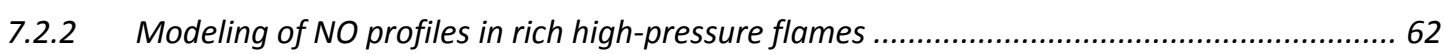

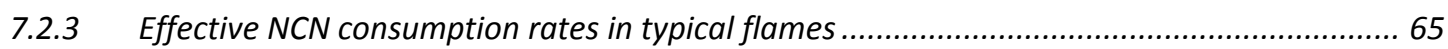

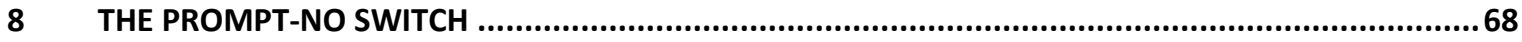

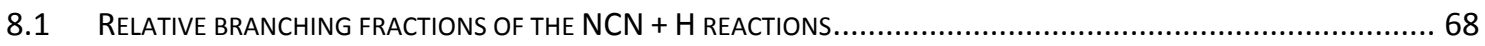

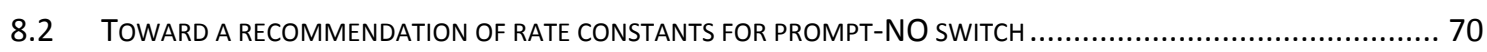

8.2.1 Influence on the overall rate constant....................................................................... 71

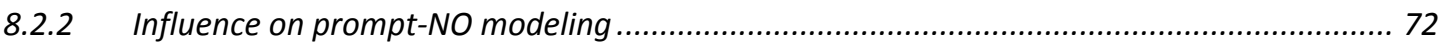

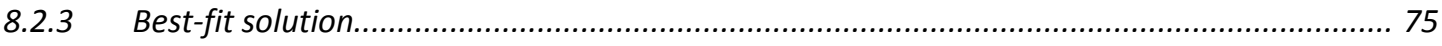

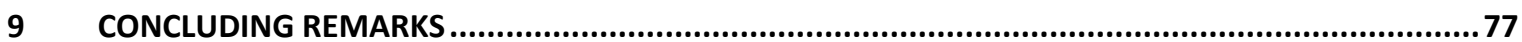




\section{Introduction}

The cyanonitrene radical (NCN), a symmetric three-atomic linear diradical with a triplet electronic ground state, is known since the early 1960s. It was spectroscopically detected and characterized in several laboratories at about the same time and served as a model example for a species with a gas phase spectrum showing distinct spin-orbit interactions and Renner-Teller effects [1-6]. Its role in combustion chemistry became manifest around the year 2000, when Moskaleva et al. [7,8] obtained theoretical evidence that the $\mathrm{CH}+\mathrm{N}_{2}$ reaction, which was shown to be crucial for the formation of prompt-NO and hence was implemented in many detailed combustion models in terms of the so-called Fenimore mechanism long before [9-11], yields $\mathrm{NCN}+\mathrm{H}$ rather than the formerly assumed products $\mathrm{HCN}+\mathrm{N}$. Albeit the original Fenimore pathway does not obey spin conservation (a doublet + singlet reaction should not directly yield singlet + quartet species as the main products) $[9,12]$ and therefore the assumed rate constant values were unusually high for such a spin-forbidden process, it was possible to reproduce experimentally observed prompt-NO formation trends [10,13]. The new pathway with $\mathrm{NCN}+\mathrm{H}$ products proposed by Moskaleva et al. [7,8] accounts for spin conservation (a doublet + singlet reaction can yield triplet + doublet species without overall spin-flip). In order to verify the role of the NCN radical as the major reaction product, to further highlight associated alternative reaction pathways resulting from possible bimolecular reactions of NCN, and to come up with a consistent mechanistic description of prompt-NO formation, a considerable number of experimental and theoretical studies have been devoted to the $\mathrm{CH}+\mathrm{N}_{2}$ reaction and the kinetics and thermodynamics of NCN since then. The aim of the present work is to review and discuss these studies to provide a comprehensive picture of the current state of knowledge on the spectroscopy and thermodynamics of NCN, as well as on the kinetics of its formation and consumption reactions with emphasis on combustion-relevant conditions. Moreover, the influence of kinetic and thermodynamic data of NCN on the modeling of prompt-NO formation will be examined.

The overall kinetics of the $\mathrm{CH}+\mathrm{N}_{2}$ reaction is complicated because this bimolecular step initiates a multi-channel/multi-well reaction system including a doublet-quartet spin conversion. A schematic energy diagram illustrating the essential features based on data compiled from Refs. [7,14-19] is shown in Fig. 1. We will refer to and further discuss the details of this key figure throughout the review at different places. Nowadays, it is well known that the kinetic behavior of such a complex-forming bimolecular reaction is governed by the competition between reactive steps and collisional energy transfer (see e.g. Ref. [11]). As indicated above, in the case of $\mathrm{CH}+\mathrm{N}_{2}$ the occurrence of a non-adiabatic transition between different spin states leads to additional complications. These so-called intersystem crossing (ISC) processes are known to be comparatively slow, and therefore reaction channels proceeding along such pathways are termed to be "spin-forbidden" [20]. 
For an adequate treatment of this reaction system at combustion-relevant conditions, it is necessary to first consider the reaction $\mathrm{CH}+\mathrm{N}_{2}$ leading to $\mathrm{NCN}+\mathrm{H}$ on the doublet surface or, via ISC, to $\mathrm{HCN}+$ $\mathrm{N}$ on the quartet surface. But then, it is also necessary to account for the consecutive bimolecular reactions $\mathrm{NCN}+\mathrm{H}$ leading either back to $\mathrm{CH}+\mathrm{N}_{2}$, again on the doublet surface, or forward toward $\mathrm{HCN}+\mathrm{N}$ on the quartet surface.

Obviously, the specification of the spin states of the involved species is important - all the more as the NCN radical exhibits a low-lying first electronically excited singlet state (Section 2). At several points of this review, it will turn out to be important to make a clear distinction between the NCN triplet electronic ground state, termed ${ }^{3} \mathrm{NCN}$, and the first electronically excited singlet state, termed ${ }^{1} \mathrm{NCN}$. This of course holds true for the spectroscopic and thermodynamics properties but also for the reactivity of the two species proceeding on separate potential energy surfaces (Section 5). In the literature, however, "NCN" typically refers to ${ }^{3} \mathrm{NCN}$ and reported rate constant expressions for "NCN" reactions either correspond to an appropriate thermal average over the contributions of both spin states or, in the majority of cases, simply assume that possible ${ }^{1} \mathrm{NCN}$ contributions can be neglected. In the following, we will adopt the notation "NCN" for ${ }^{3} \mathrm{NCN}$ or a (presumably) ${ }^{3} \mathrm{NCN}$-dominated overall reaction. However, whenever essential for the respective discussion and in the rare cases where authors have distinguished between ${ }^{1} \mathrm{NCN}$ and ${ }^{3} \mathrm{NCN}$ reactivity, we will specify the corresponding spin states to avoid erroneous attribution. The same holds true for other species such as ${ }^{2} \mathrm{CH}$, where we explicitly state the spin state only when necessary for understanding.

Back to the basic characteristics of the reaction $\mathrm{CH}+\mathrm{N}_{2}$ as outlined in Fig. 1. Under conditions where collisional stabilization of the intermediates is negligible (or in steady-state), the overall reaction system can be expressed in terms of the following bimolecular reactions (with reversibility written explicitly only if relevant under typical combustion conditions, see below):

$$
\begin{aligned}
& \mathrm{CH}+\mathrm{N}_{2} \rightleftharpoons \mathrm{NCN}+\mathrm{H} \\
& \mathrm{CH}+\mathrm{N}_{2} \rightarrow \mathrm{HCN}+\mathrm{N} \\
& \mathrm{NCN}+\mathrm{H} \rightarrow \mathrm{HCN}+\mathrm{N}
\end{aligned}
$$

It turns out that the subtle interplay between these three reactions has a most pronounced influence on the kinetics of prompt-NO formation, and we will dedicate a considerable part of this review to this problem. Moreover, we will introduce the technical term prompt-NO switch to highlight the crucial role of the branching between the major reaction channels of $\mathrm{NCN}+\mathrm{H}$, namely the reverse reaction (R-1a) and the forward reaction (R2), to properly describe the further fate of the NCN radical in combustion 
systems. Here, forward is meant in a sense that reaction (R2) essentially corresponds to the traditional Fenimore initiation step on the route to $\mathrm{NO}$ by an effective transformation of $\mathrm{CH}+\mathrm{N}_{2}$ to $\mathrm{HCN}+\mathrm{N}$.

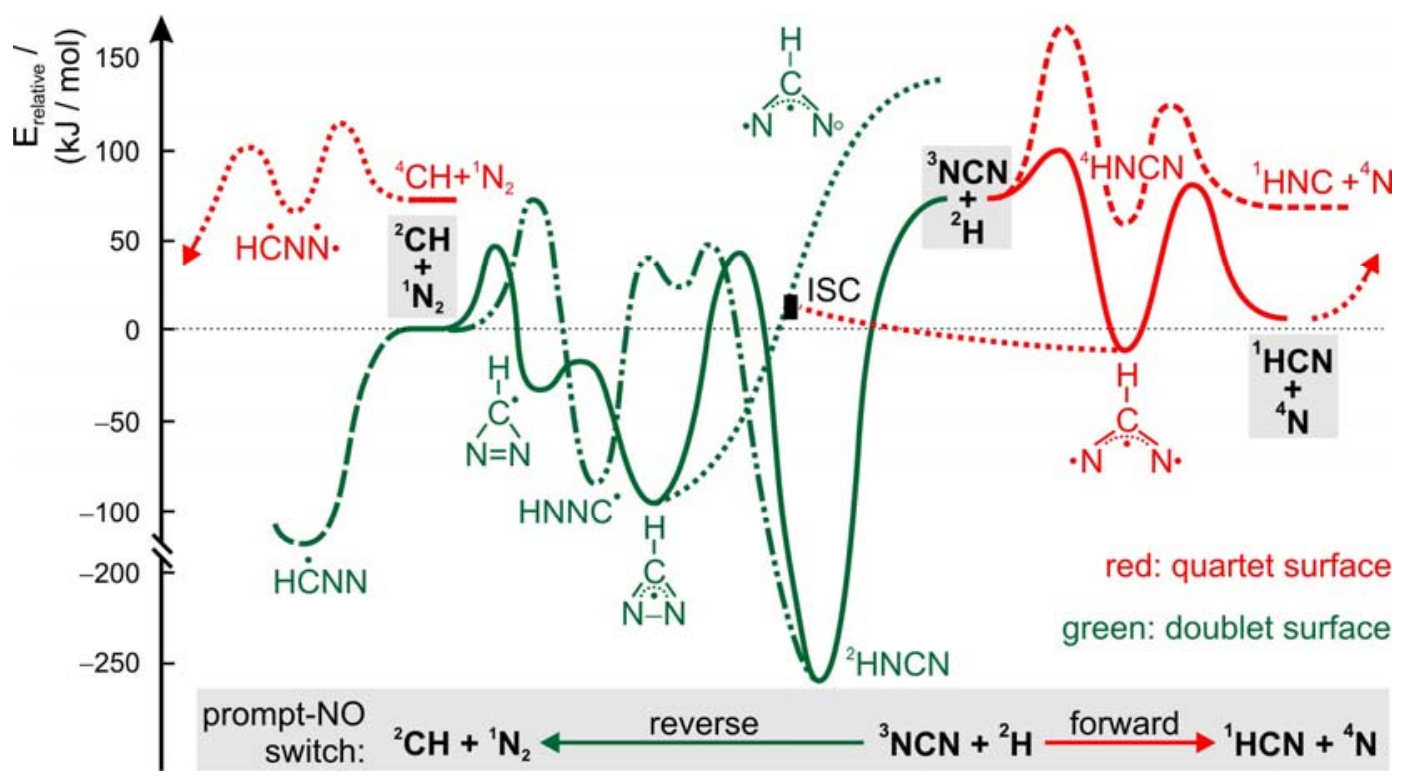

Fig. 1. Schematic potential energy diagram of the $\mathrm{CHN}_{2}$ reaction system, illustrating the close relationship of the spin-allowed prompt-NO initiation reaction $\mathrm{CH}+\mathrm{N}_{2}$ yielding $\mathrm{NCN}+\mathrm{H}$ and the subsequent prompt-NO switch reaction $\mathrm{NCN}+\mathrm{H}$ yielding either the reverse $\mathrm{CH}+\mathrm{N}_{2}$ or the forward $\mathrm{HCN}+\mathrm{N}$ products (prompt-NO switch). Approximate energies, molecular structures, and reaction paths have been compiled from Refs. [7,14-17]. The solid curves represent the most important, the other curves minor pathways. The green and red dotted curves indicate the formerly considered spin-forbidden prompt-NO initiation pathway $\mathrm{CH}+\mathrm{N}_{2} \rightarrow \mathrm{HCN}+\mathrm{N}$, where the approximate energy of the ISC region that connects the doublet and quartet potential energy surfaces was adopted from Refs. $[18,19]$. The different channels are discussed in Sections 6 and 7.

A first attempt to kinetically describe the $\mathrm{CH}+\mathrm{N}_{2}$ reaction system on the basis of Rice-RamspergerKassel-Marcus (RRKM) theory dates back to 1983, when Berman and Lin [21] analyzed their experimental results obtained in a slow-flow reactor. The authors were able to model the pressure dependence of the rate constant between 3.3 and $105 \mathrm{kPa}$ at a temperature of $297 \mathrm{~K}$ and the temperature dependence between 297 and $675 \mathrm{~K}$ at a pressure of $13.3 \mathrm{kPa}$. It was assumed that the reaction proceeds via a long-lived intermediate, $\mathrm{HCN}_{2}$ (with a hypothetical open-chain $\mathrm{HCNN}$ structure) that is stabilized in the low-temperature experiments, whereas at higher temperatures, above $1000 \mathrm{~K}$, the direct abstraction/metathesis reaction giving $\mathrm{HCN}+\mathrm{N}$ dominates. The doublet-quartet spin transition was assumed to be fast due to multiple curve crossings possible in the long-lived complex.

In 1996, Fulle and Hippler [22] performed similar experiments and used an empirical Troe fit to describe the experimentally obtained temperature and pressure dependence of the rate constant for $\mathrm{CH}$ $+\mathrm{N}_{2}$ in the ranges $T=200-715 \mathrm{~K}$ and $p=0.1-15 \mathrm{MPa}$, respectively. Moreover, the high-temperature limiting value of the rate constant was estimated with the simplified statistical adiabatic channel model. 
Similar to Berman and Lin [21] and later Moskaleva and Lin [23], the authors concluded that collisional stabilization of $\mathrm{HCN}_{2}$ is dominant at low temperatures whereas dissociation of this adduct becomes important at higher temperatures. Similar theoretical analyses were performed at about the same time by Rodgers and Smith [24] and Miller and Walch [25]. Note that two structures of $\mathrm{HCN}_{2}$ described earlier in the literature [26-29] were considered an open-chain dative structure, $\mathrm{H}-\mathrm{C}-\mathrm{N}-\mathrm{N}$, and a structure containing a three-membered $\mathrm{CN}_{2}$ ring, $\mathrm{H}-\mathrm{CN}_{2}$.

The electronic non-adiabaticity of reaction (R1b) was for the first time explicitly addressed in a theoretical work by Cui et al. [18] in 1999. The authors, in a one-dimensional approach, used the distorted wave approximation to calculate the ISC probability. The value for the spin-orbit coupling element was obtained from ab initio calculations, and the crossing seam of the potential energy surfaces was treated as a transition state. The predicted rate constant was found to be two orders of magnitude below the experimental values. Interestingly, this disagreement was discussed in terms of the approximations introduced in the theoretical treatment rather than the possibility that additional, not yet considered, reaction channels may dominate the overall reaction.

Shortly afterwards, in a seminal theoretical work published in 2000, Moskaleva and Lin [7] showed that $\mathrm{NCN}+\mathrm{H}$, and not as previously assumed $\mathrm{HCN}+\mathrm{N}$, are the major products of the $\mathrm{CH}+\mathrm{N}_{2}$ reaction at temperatures relevant for prompt-NO formation $(T>1200 \mathrm{~K})$. This reaction, (R1a), proceeds on the doublet surface observing spin conservation and is faster than reaction (R1b) that leads to the spinforbidden products $\mathrm{HCN}+\mathrm{N}$ with the $\mathrm{N}$ atom in its quartet electronic ground state. This new spinconserved pathway has been also confirmed on CASPT2 level by Takayanagi [30]. On the basis of molecular data from quantum chemical calculations (mainly at G2M(RCC) level of theory), the overall rate constant of reaction (R1a) was calculated by Moskaleva and Lin with a multichannel variational RRKM approach. The values obtained were found to be consistent with available results from previous shock tube experiments $(T>2000 \mathrm{~K})$.

The first clear experimental evidence for $\mathrm{NCN}$ being the major product of the $\mathrm{CH}+\mathrm{N}_{2}$ reaction was provided in a shock tube study by Vasudevan et al. in 2007 [31]. They monitored $\mathrm{CH}$ and NCN by laser absorption and obtained temperature-dependent rate constants $k_{1 \mathrm{a}}(T)$ and relative branching fractions $k_{1 \mathrm{a}} /\left(k_{1 \mathrm{a}}+k_{1 \mathrm{~b}}\right)$, int the $2228-2905 \mathrm{~K}$ temperature range. Also a first estimate of the high-temperature absorption coefficient of NCN could be provided. The reported temperature-dependent overall rate constants of reaction (R1a) are in good agreement with values determined earlier by Dean et al. [32] in similar shock tube experiments following perturbations of the $\mathrm{CH}$ profiles by added $\mathrm{N}_{2}$.

A theoretical study with multi-reference ab initio methods for the electronic structure calculations and variable reaction coordinate (VRC) transition state theory (TST) for the calculation of the rate constants was published in 2008 by Harding et al. [15]. The authors discussed that the use of multireference methods was critical for the properties of two rate-limiting transitions states and hence lead to 
a better agreement of the calculated rate constants with high-temperature shock tube results than the earlier predictions from Ref. [7]. At high temperatures, above $1940 \mathrm{~K}$, the predicted rate constants are in very good agreement with the measured values from Ref. [31] and also support values at lower temperatures near $1500 \mathrm{~K}$ used in a flame modeling study by El Bakali et al. [33].

In a further theoretical study from M.C. Lin's group in 2013, Teng et al. [16] published a rate expression corresponding to $k_{1 \mathrm{a}}$ that was obtained from master equation (ME) calculations with energyspecific rate constants from variational TST and RRKM theory. Stationary points on the potential energy surface were calculated at the coupled-cluster level of theory extrapolated to the complete basis set limit. In the context of Ref. [16], an error in the rate constant code used in Ref. [7] was revealed that lead to an underestimation of $k_{1 \mathrm{a}}$ in the said publication.

In a very recent theoretical work by Klippenstein et al. [17], the $\mathrm{CHN}_{2}$ reaction network was reanalyzed with emphasis on possible collisional stabilization effects. To model the pressure dependence of the rate constants, a rigorous ME approach was used in combination with high-level electronic structure methods for the determination of thermochemical data of NCN. It followed that collisional stabilization of HNCN (see Fig. 1) is not necessarily negligible. For kinetic modeling, pressuredependent rate constants for the range $p=0.001-10 \mathrm{MPa}$ are tabulated in the Supplementary Material of Ref. [17] in PLOG format (see e.g. Ref. [34]). Listed are the extended Arrhenius parameters for the reactions (R1a) and (R2) as well as for the reactions:

$$
\begin{aligned}
& \mathrm{CH}+\mathrm{N}_{2} \rightarrow \mathrm{HNCN} \\
& \mathrm{NCN}+\mathrm{H} \rightarrow \mathrm{HNCN} \\
& \mathrm{NCN}+\mathrm{H} \rightarrow \mathrm{HNC}+\mathrm{N}
\end{aligned}
$$

The rate constants for the corresponding reverse reactions were obtained via the equilibrium constants. In this way, the reaction network interconnecting the species $\mathrm{CH}+\mathrm{N}_{2} / \mathrm{H}+\mathrm{NCN} / \mathrm{HNCN} / \mathrm{HCN}$ $+\mathrm{N} / \mathrm{HNC}+\mathrm{N}$ was described with a mutually consistent kinetic and thermodynamic data set. In a recent review [35] on nitrogen chemistry in combustion, these data were incorporated into a recommended global mechanism.

A particular role in this context plays the enthalpy of formation of $\mathrm{NCN}, \Delta_{\mathrm{f}} H^{\circ}(\mathrm{NCN})$. Because reaction (R1a) is endothermic and the $\mathrm{H}+\mathrm{NCN}$ combination reaction is barrierless, $\Delta_{\mathrm{f}} H^{\circ}(\mathrm{NCN})$ critically determines the kinetic data of the reversible reaction pair (R1a)/(R-1a) and, as a consequence, strongly influences the branching ratios between reaction (R-1a) and other NCN-consuming reactions that may irreversibly remove NCN from the system. This competition has a large effect on the overall kinetics of prompt-NO formation. The reliable determination of $\Delta_{\mathrm{f}} H^{\circ}(\mathrm{NCN})$ turned out to be not a 
straightforward task, and the actual value was the subject of a long-standing debate, which has been resolved only in recent years (see e.g. Refs. [15,17,36,37]).

Before actual measurements of NCN in flames could be accomplished, a number of challenges had to be met. First, it was necessary to characterize spectroscopic properties of NCN at high temperatures, in particular absorption cross-sections to enable absolute concentration measurements. Furthermore, it was necessary to develop techniques that were sufficiently sensitive to reliably detect the expected low concentrations of NCN but also sufficiently selective to monitor $\mathrm{NCN}$ in presence of interfering species such as NH. The first convincing experimental evidence for the occurrence of NCN in flames was provided by Smith in 2003 [38]. On the basis of an earlier work [39], the author was able to determine the profiles of NCN concentration (in relative values) as function of the height above the burner (HAB) in low-pressure $\mathrm{CH}_{4} / \mathrm{O}_{2} / \mathrm{N}_{2}$ and $\mathrm{CH}_{4} / \mathrm{N}_{2} \mathrm{O} / \mathrm{N}_{2}$ flames by laser-induced fluorescence (LIF), adopting the $\tilde{\mathrm{A}}-\tilde{\mathrm{X}}$ transition of NCN near a wavelength of $329 \mathrm{~nm}$. By comparing the measured NCN profiles with $\mathrm{CH}$ profiles from Berg et al. [40] in a rich $\mathrm{CH}_{4} / \mathrm{O}_{2} / \mathrm{N}_{2}$ flame, Smith was also able to show that $\mathrm{NCN}$ is likely to be produced in the $\mathrm{CH}+\mathrm{N}_{2}$ reaction and indeed represents a critical intermediate in promptNO formation. Subsequently, this approach via LIF was further developed and extended to different types of flames [41-46]. The quantification in absolute mole fractions of NCN required the use of cavity ring-down spectroscopy (CRDS), a highly sensitive absorption technique. It was first implemented to measure the absolute mole fraction of NCN in a low-pressure flame by Lamoureux et al. [42].

The analysis of these increasingly detailed measurements, however, required, besides hightemperature spectroscopic data, in particular adequately parameterized chemical mechanisms. Even though in some earlier works, mechanisms containing a number of NCN reactions already had been used ([47], for a review see [48]), a systematic inclusion of NCN reactions in kinetic combustion mechanisms started only around the year 2005 ([33,44,49-52], for a review see [35]). Soon after, it became obvious that for these reactions more reliable high-temperature thermodynamic and kinetic data would be required. The rather high uncertainty or even the lack of these data has stimulated a considerable number of experimental and theoretical studies on NCN elementary chemical steps in subsequent years.

It the present work, we review and discuss the most crucial points within this ongoing story of NCN and its role in combustion. In structuring this article, we diverge, however, from the historical outline just given and choose a more deductive way. We commence with molecular and spectroscopic properties of NCN and proceed via thermodynamic and kinetic data of NCN elementary reactions toward important global-kinetic modeling aspects at flame conditions.

To begin with, Section 2 deals with fundamental spectroscopic properties of NCN. In particular, high-temperature absorption and fluorescence spectra will be discussed with emphasis on the determination of absorption cross sections for quantitative NCN detection. Based on the spectroscopic 
data, the literature values for the heat capacity and the enthalpy of formation of NCN are collected, compared, and discussed in Section 3. In Section 4, the production of NCN to perform gas phase kinetic experiments is elucidated, and cyanogen azide, $\mathrm{NCN}_{3}$, is identified and characterized as a suitable photolytic and thermolytic precursor. In this context, the problem of NCN spin states and in particular the molecular mechanisms of their interconversion become relevant. These problems are discussed in Section 5 before the current state of the kinetics of NCN elementary chemical steps is reviewed in detail in Section 6. In the subsequent two Sections, 7 and 8, the spectroscopic, thermodynamic, and kinetic data are used to examine absolute NCN concentrations as well as mechanistic aspects of NCN production and consumption in flames.

Along these lines, in Section 7, the measurement and modeling of NCN in flames are reviewed and discussed. Problems with absolute calibration of NCN concentrations are examined, and a combination of LIF with CRDS is proposed as one approach to circumvent these difficulties. The results of these measurements, that is essentially species concentration profiles as function of the HAB, are compared to simulation results, and extensive sensitivity analyses are used to determine the significance of selected NCN reactions. On the basis of these analyses, the influence of the reaction system (R1a)-(R4) is then studied in more detail in Section 8. Particular emphasis is put on the subtle temperature-dependent switch between the NCN-consuming reactions (R-1a) and (R2), proceeding on the doublet and quartet potential energy surfaces, respectively. A simple two-parameter description of this switching behavior is proposed and optimized by simulating $\mathrm{NCN}, \mathrm{HCN}$, and $\mathrm{NO}$ profiles in a rich low-pressure $\mathrm{CH}_{4} / \mathrm{O}_{2} / \mathrm{N}_{2}$ flame. Then, the selected two parameters are used for simulating the species profiles obtained in five low-pressure flames and for the NO prediction in high-pressure flames. General conclusions are drawn and recommendations for the modeling of prompt-NO formation are given.

Finally, in Section 9, some general conclusions are drawn.

Before concluding this introduction section, we note that other prompt-NO initiation steps that will not be discussed in this review may also contribute to $\mathrm{NCN}$ formation to a minor extent. But reactions such as $\mathrm{C}+\mathrm{N}_{2}[32,53],{ }^{1,3} \mathrm{CH}_{2}+\mathrm{N}_{2}$ [54-56], $\mathrm{C}_{2}+\mathrm{N}_{2}[57,58]$, and $\mathrm{C}_{2} \mathrm{O}+\mathrm{N}_{2}[49,59,60]$ appear to exhibit high activation barriers or result in low net fluxes due to low concentrations of the relevant intermediates under most flame conditions [35]. Both reactions, $\mathrm{C}_{2} \mathrm{O}+\mathrm{N}_{2} \rightarrow \mathrm{NCN}+\mathrm{CO}$ and $\mathrm{C}+\mathrm{N}_{2}(+\mathrm{M}) \rightarrow \mathrm{NCN}$ $(+\mathrm{M})$, have been discussed to feed into the NCN radical pool [35,44,49,59], but a detailed inspection of the related potential energy surfaces showed that they cannot compete with the $\mathrm{CH}+\mathrm{N}_{2}$ reaction. $\mathrm{Ab}$ initio calculations performed by Zhu et al. [60] on the $\mathrm{C}_{2} \mathrm{O}+\mathrm{N}_{2}$ reaction gave activation barriers of 253 $\mathrm{kJ} \mathrm{mol}^{-1}$ and $280 \mathrm{~kJ} \mathrm{~mol}^{-1}$ for the minor NCN formation pathways on the singlet and triplet energy surface, respectively. These values are too high for these reactions to be of any importance under practical combustion conditions. Also the main reaction channel of the $\mathrm{C}_{2} \mathrm{O}+\mathrm{N}_{2}$ reaction, yielding $\mathrm{CN}_{2}$ $+\mathrm{CO}$, is too slow. On the other hand, the reaction $\mathrm{C}+\mathrm{N}_{2}(+\mathrm{M}) \rightarrow \mathrm{NCN}(+\mathrm{M})$ is the reverse of the unimolecular decomposition of the NCN radical, which will be discussed in some detail in Section 6 . 
But instead of yielding NCN radicals, the bimolecular product channel $\mathrm{N}+\mathrm{CN}$ appears to be the major reaction path at high temperatures [53], whereas recombination to $\mathrm{CN}_{2}$ is likely to prevail only at low temperatures $[61,62]$. Though some of these reactions might gain influence on prompt-NO formation under special conditions, their general importance is probably small, and we do not discuss them in more detail within this review.

\section{Spectroscopic fundamentals for quantitative detection of NCN}

\subsection{Electronic energy levels}

Quantitative detection of NCN radicals in flames and in kinetics experiments has been accomplished in the UV range by both laser absorption and laser-induced fluorescence. Fig. 2 illustrates the energy level diagram of the relevant NCN singlet and triplet manifolds. The molecule is linear in the lowest triplet and singlet states as well as in the indicated higher electronic states $[2,63]$. The term symbols of the lowest electronic states are $\tilde{\mathrm{X}}^{3} \Sigma_{\mathrm{g}}^{-}, \tilde{\mathrm{a}}^{1} \Delta_{\mathrm{g}}$, and $\tilde{\mathrm{b}}{ }^{1} \Sigma_{\mathrm{g}}^{+}$. The metastable ã and $\tilde{\mathrm{b}}$ states lie at $(8146 \pm 80)$ $\mathrm{cm}^{-1}$ and $(13139 \pm 80) \mathrm{cm}^{-1}$ above the $\tilde{\mathrm{X}}^{3} \Sigma_{\mathrm{g}}^{-}$ground state as was deduced from photoelectron spectra of the $\mathrm{NCN}^{-}$anion [64].

At room temperature and for spectroscopic purpose, NCN radicals are often generated by flash photolysis of cyanogen azide $\left(\mathrm{NCN}_{3}\right)$, yielding transient $\mathrm{NCN}$ in both its singlet and its triplet state [5]. The electronic transitions between the ground state $\tilde{X}$ and the first metastable states $\tilde{a}$ and $\tilde{b}$ are both dipole- and spin-forbidden [65]. Accordingly, the consecutive occurrence of NCN in the ã and $\tilde{X}$ states in one experiment has to be attributed to ISC during $\mathrm{NCN}_{3}$ decomposition and/or in the generated $\mathrm{NCN}$ radical itself. Production schemes for NCN as well as the problem of singlet-triplet relaxation will be discussed in detail in Sections 4 and 5.2. 

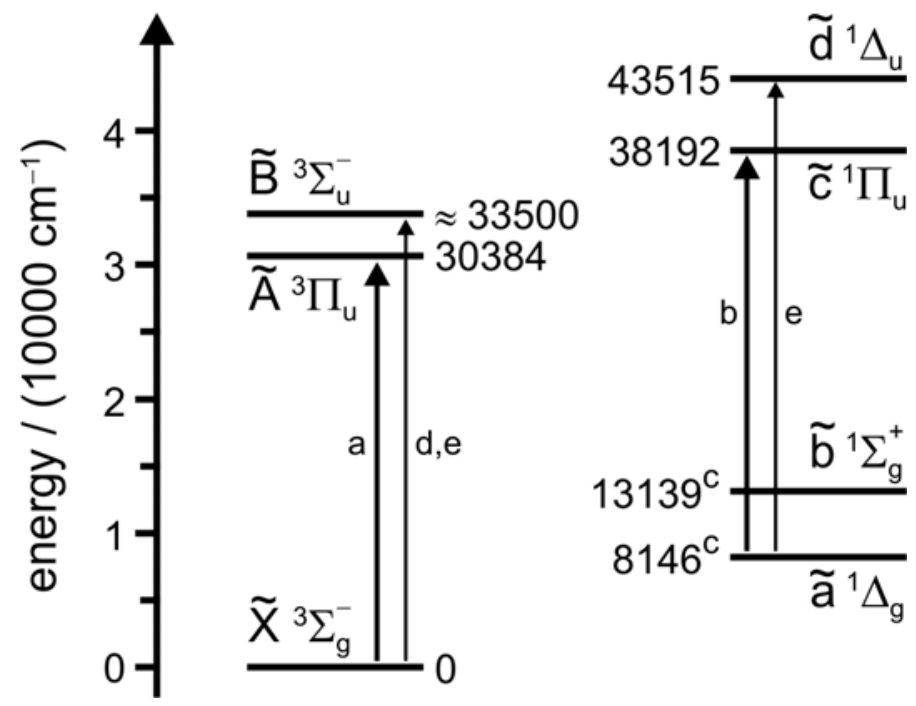

Fig. 2. Energy level diagram of singlet and triplet manifolds of NCN compiled from Refs. [2] $]^{\mathrm{a}},[63]^{\mathrm{b}},[64]^{\mathrm{c}}$, $[66]^{\mathrm{d}},[67]^{\mathrm{e} ;}$ given are the term values $T_{0}$ that is, including zero-point vibrational energy.

Electronic spectra of NCN are complex, exhibiting strong Renner-Teller effects, and are characterized by vibronic structure and branches comprising of a large number of overlapping rotational lines. The strong $\tilde{\mathrm{A}}^{3} \Pi_{\mathrm{u}} \leftarrow \tilde{\mathrm{X}}^{3} \Sigma_{\mathrm{g}}^{-}$band and the $\tilde{\mathrm{c}}^{1} \Pi_{\mathrm{u}} \leftarrow \tilde{\mathrm{a}}^{1} \Delta_{\mathrm{g}}$ band of NCN have been first observed by Jennings and Linnett [1] (tentatively attributed to $\mathrm{CN}_{2}$ ) and Kroto [5]. Soon after, the rotationally resolved bands were assigned by Herzberg and Travis [2] and Kroto [63], yielding (000) - (000) transitions at $30383.74 \mathrm{~cm}^{-1}(329.123 \mathrm{~nm})$ and $30045.76 \mathrm{~cm}^{-1}(332.826 \mathrm{~nm})$ for the $\tilde{\mathrm{A}}-\tilde{\mathrm{X}}$ and $\tilde{\mathrm{c}}-\tilde{\mathrm{a}}$ systems, respectively. Additional triplet and singlet bands have been identified based on their vibrational structure by Kroto et al. [67] and were assigned to the $\widetilde{\mathrm{B}}{ }^{3} \Sigma_{\mathrm{u}}^{-} \leftarrow \tilde{\mathrm{X}}^{3} \Sigma_{\mathrm{g}}^{-}$system with a (000) $-(000)$ transition at about $33512 \mathrm{~cm}^{-1}(298.40 \mathrm{~nm})[66,67]$ and the $\tilde{\mathrm{d}}^{1} \Delta_{\mathrm{u}} \leftarrow \tilde{\mathrm{a}}^{1} \Delta_{\mathrm{g}}$ system with a $(000)-(000)$ transition at about $35369 \mathrm{~cm}^{-1}(282.73 \mathrm{~nm})[66]$.

\section{$2.2{ }^{3} \mathrm{NCN}$ spectroscopy at room temperature}

Inspection of the literature shows that after the work of Herzberg and Travis [2], NCN spectroscopy had received relatively little attention and, in most cases, set a focus on the triplet state. An original absorption spectrum of NCN taken from the seminal work [2] is reprinted in Fig. 3.

Herzberg and Travis analyzed rotational transitions in the three subbands of the (000) - (000) band and partially of the (010) - (010) band. They also showed the existence of a Renner-Teller interaction, where the situation is complicated by a non-negligible spin-orbit coupling. A schematic vibrational energy diagram of NCN obtained from a more recent simulation by Lamoureux et al. [68] is shown in Fig. 4. 


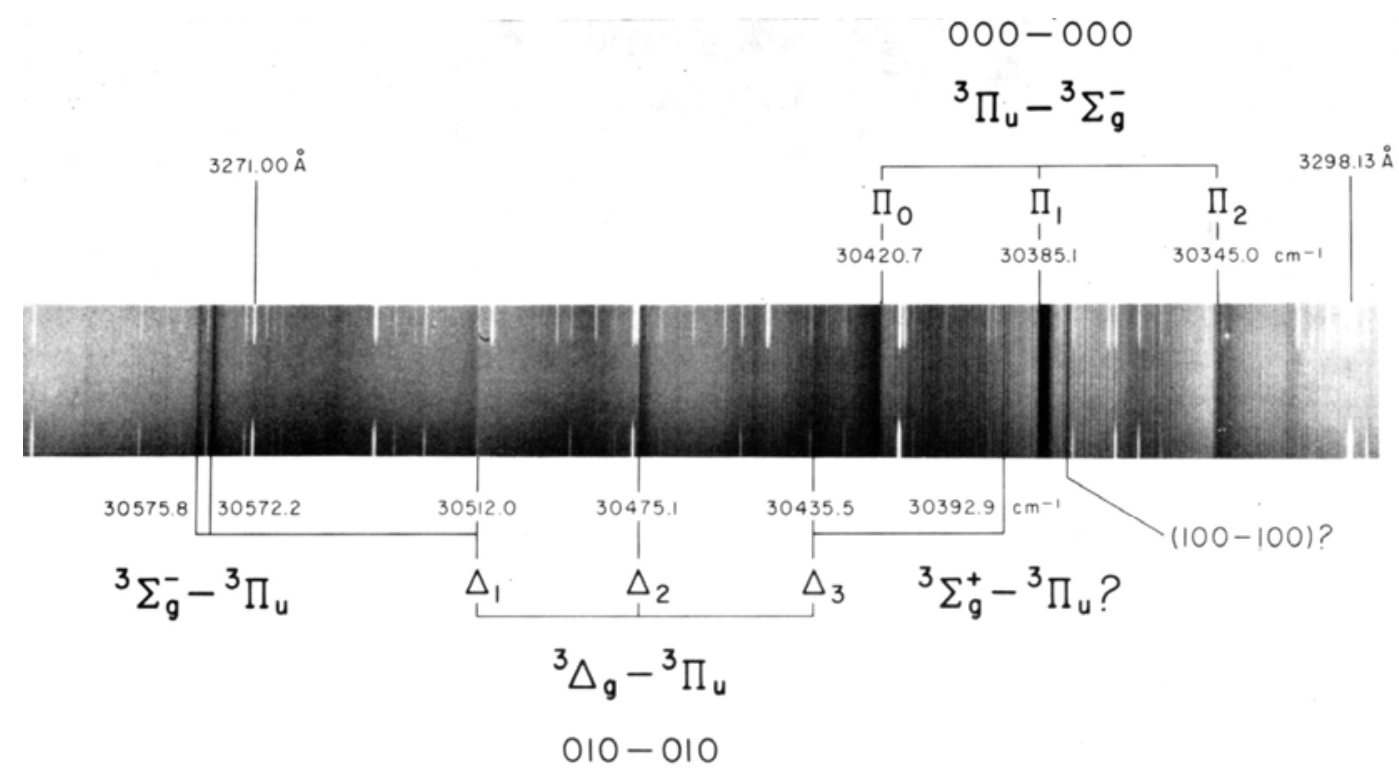

Fig. 3. The absorption spectrum of ${ }^{3} \mathrm{NCN}$, showing the proposed vibronic assignments given by Herzberg and Travis [2]. Reprinted from Canadian J. Physics, 42, Herzberg and Travis, pp. 1658-1675, (C 1964, with permission from Canadian Science Publishing.

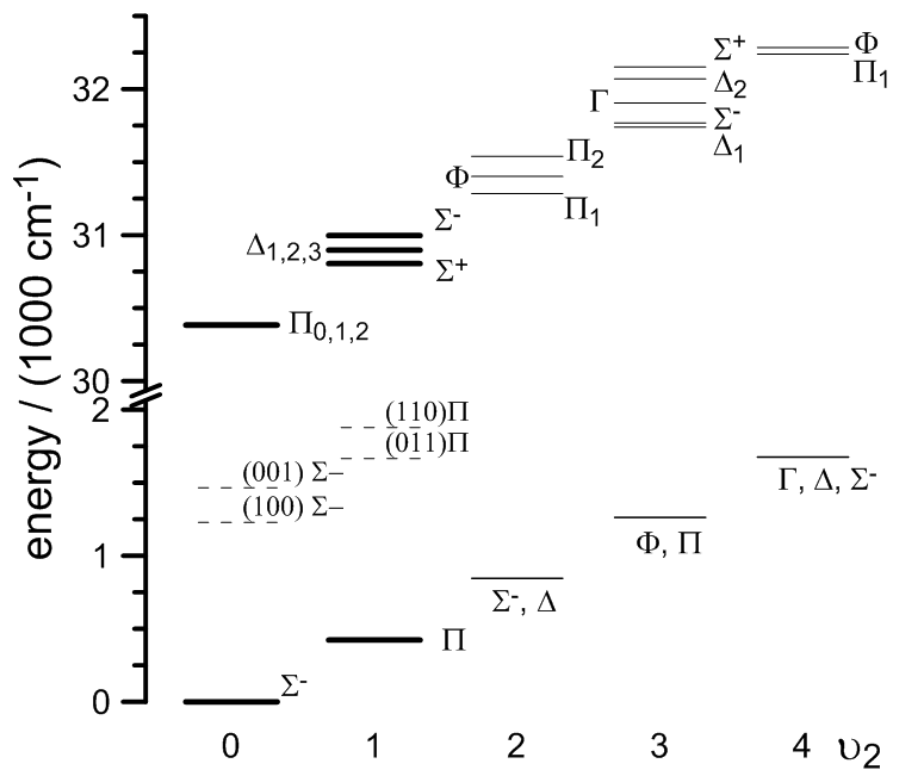

Fig. 4. Energy levels $\left(0 \mathrm{v}_{2} 0\right)$ within the $\tilde{\mathrm{A}}-\tilde{\mathrm{X}}$ band in ${ }^{3} \mathrm{NCN}$ (as a function of the bending vibrational quantum number, $\mathrm{v}_{2}$ ); levels in bold were initially included in the simulation of Ref. [42]; additional levels with excited stretching vibrations are also indicated (short-dashed lines); note that all the levels shown were included in the simulated spectrum reported in Ref. [68].

The LIF excitation spectra of NCN at $300 \mathrm{~K}$ recorded by Smith et al. [39] allowed the identification of spin-orbit triplets. Beaton et al. $[69,70]$ confirmed the assignments of the $(000)-(000)$ and the $(010)$ 
-(010) bands in the near UV and provided a more detailed analysis of the rotational structure from their high resolution $\left(0.06 \mathrm{~cm}^{-1}\right) \mathrm{LIF}$ spectra. It was possible to improve molecular parameters compared to those reported previously by Herzberg and Travis [2]. Perić et al. [71] performed ab initio calculations on NCN, focusing on the Renner-Teller effects in the excited states. The NCN band head was spectrally resolved by narrow-bandwidth $\left(<0.01 \mathrm{~cm}^{-1}\right)$ laser absorption measurements at $293 \mathrm{~K}$ by Dammeier and Friedrichs [72]. Hetzler and Olzmann [73] measured a LIF excitation spectrum of NCN at $293 \mathrm{~K}$ with a spectral bandwidth of $1 \mathrm{~cm}^{-1}$. The spectrum was obtained at saturated LIF conditions and is shown in Fig. 5. The authors observed the triplet structure in the $\tilde{\mathrm{A}}^{3} \Pi_{\mathrm{u}}(000)-\tilde{\mathrm{X}}^{3} \Sigma_{\mathrm{g}}^{-}(000)$ band and in the two components $\Sigma-\Pi$ and $\Delta-\Pi$ of the $(010)-(010)$ bands as they are assigned in Fig. 3. Additional lines around $317-320 \mathrm{~nm}$ corresponding to the $(020)-(000)$ and $(030)-(010)$ bands were also observed (see Supplementary Material of Ref. [73] and Fig. 6).

The simulation of well resolved room temperature LIF spectra with the spectral simulation program PGOPHER [74] served as a critical test of the capability to reproduce the complex vibrational structure of the NCN radical. In an initial work from 2009 [42], the simulation was restricted to three vibrational features ((000) - (000) band and two components of the (010) - (010) band, shown in bold in Fig. 4), where molecular spectroscopic parameters were adopted from Beaton et al. [69,70]. As explained in Ref. [42], a few minor adjustments of the originally reported spectroscopic constants were necessary in order to fit the experimental spectra reported in Refs. [39,69,70]. Fig. 5 presents the comparison of the experimental LIF excitation spectrum from Hetzler and Olzmann [73] with simulated spectra from the two different spectroscopic models published in 2009 [42] and 2013 [68]. For the 2013 version, many additional bands such as the (020) - (020) transitions between 325.7 and $325.9 \mathrm{~nm}$ were included to further improve the simulation of spectral features that are weak at room temperature but more intense at higher temperatures.

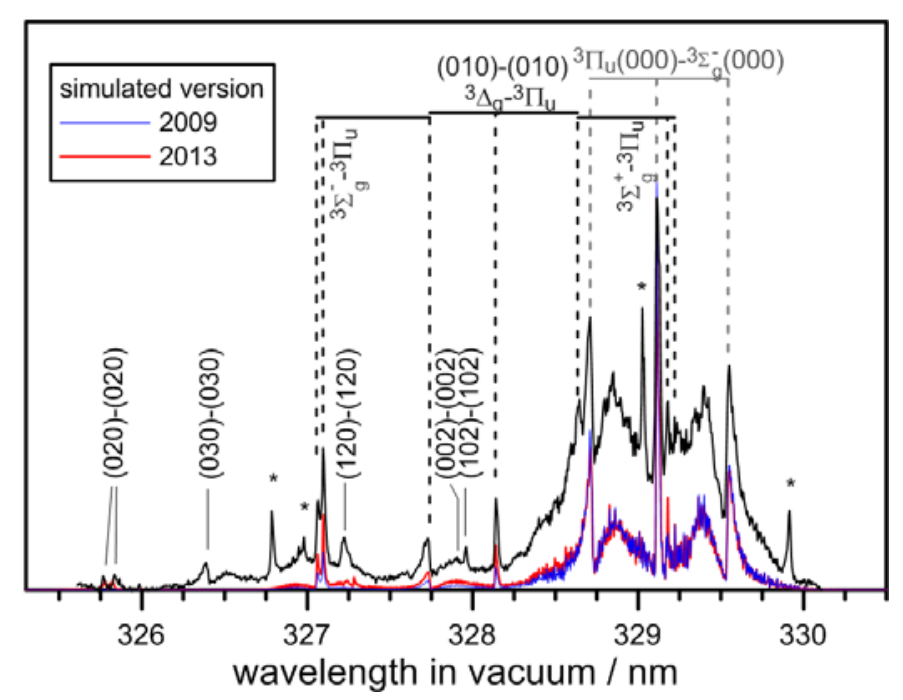

Fig. 5. LIF excitation spectra of ${ }^{3} \mathrm{NCN}$ at $293 \mathrm{~K}$ : experimentally obtained by saturated LIF [73] (black) and simulated (convoluted with a Gaussian linewidth of $0.6 \mathrm{~cm}^{-1}$ ) with PGOPHER by employing the parameter set used in Ref. [42] (blue) and in Ref. [68] (red); the symbol * marks unassigned transitions. 
Some off-diagonal excitation transitions $\left(\Delta \mathrm{v}_{2} \neq 0\right)$ were also observed by LIF at room temperature $[39,73]$ as for instance the transitions of the $(020)-(000)$ band near 317.1 and $319.7 \mathrm{~nm}$ as shown in Fig. 6. Good spectra were also obtained after exciting $\mathrm{NCN}$ along the $\Pi_{1,2}(020)-\Sigma_{\mathrm{g}}^{-}(000)$ band with the LIF signal being collected along the $\Pi_{1,2}(020)-\Sigma_{\mathrm{g}}{ }^{-}(020)$ band centered at $325.8 \mathrm{~nm}$ [73]. However, because of the weaker fluorescence intensity of the off-diagonal transitions, and the close vicinity of spectral signatures from numerous other species $(\mathrm{CH}, \mathrm{OH}, \mathrm{NCO}$, etc., see Ref. [75]), NCN detection after exciting off-diagonal transitions is not recommended in flames.

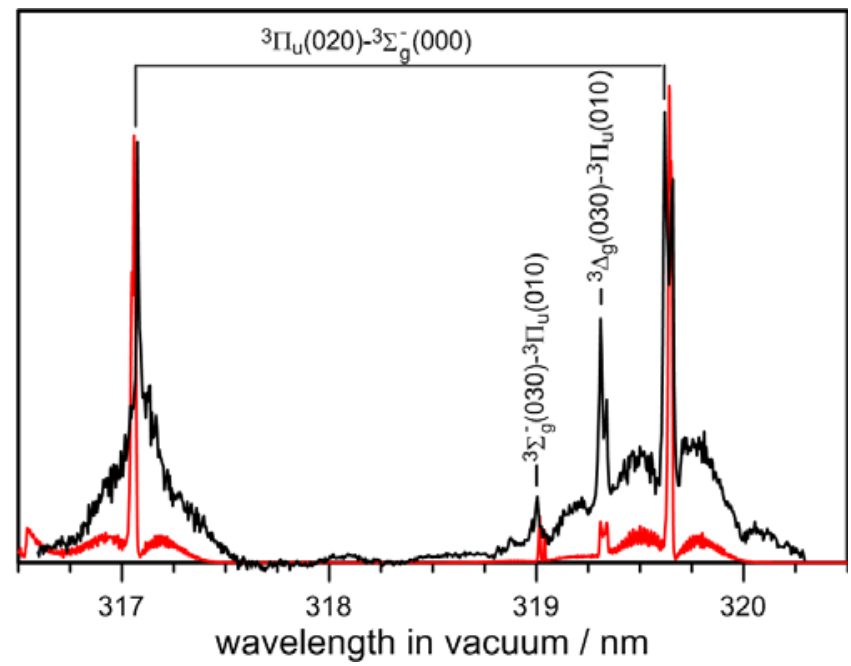

Fig. 6. Experimental saturated LIF room temperature excitation spectrum of ${ }^{3} \mathrm{NCN}$ (black) [73] revealing offdiagonal transitions in comparison with a PGOPHER simulation (red) based on the spectroscopic parameters reported in Ref. [68].

\section{$2.3 \quad{ }^{3} \mathrm{NCN}$ spectroscopy at high temperatures}

At flame temperature, it is likely that in addition to the $\Sigma_{\mathrm{g}}^{-}(000)$ state, the $\Pi_{\mathrm{u}}(010)$ state (with an energy of $423 \mathrm{~cm}^{-1}$ above the vibrational ground state, Fig. 4) is populated, giving rise to two additional vibrational hot bands, $\Sigma_{\mathrm{g}}^{-}(010)-\Pi_{\mathrm{u}}(010)$ and $\Delta_{\mathrm{g}}(010)-\Pi_{\mathrm{u}}(010)$. Based on an excitation LIF spectrum obtained in a flame at $T=1660 \mathrm{~K}$ (Fig. 7), the spectroscopic dataset of NCN has been investigated in Refs. $[42,68]$. The relative line intensities were found unaffected when varying the laser intensity within a factor of 4 , hence the spectrum was recorded in a near-linear LIF regime. Accordingly, the experimental spectrum can be used for testing the spectral simulation, both with respect to line positions and intensities. As can be seen in the inset of Fig. 7, by considering the $(000)-(000)$ and $(010)-(010)$ vibrational bands only, the simulated spectrum calculated with PGOPHER already satisfactorily fits the 
experimental LIF excitation spectrum at high temperature around the most intense band head at $\lambda=329$ $\mathrm{nm}$. However, the simulated spectrum with the 2009 data (blue trace) fails to reproduce the experimental LIF spectrum at wavelengths shorter than $328.8 \mathrm{~nm}$. Since the wavenumber of the bending vibration in the ground state is lower than those of the stretching vibrations $\left(\sim 23 \mathrm{~cm}^{-1}\right.$ compared to $\sim 1245 \mathrm{~cm}^{-1}$ and $\sim 1472 \mathrm{~cm}^{-1}$, cf. Table 1), in particular the $\left(0 v_{2} 0\right)-\left(0 v_{2} 0\right)$ bands with $v_{2}$ up to 4 have to be included in the simulation as well as some other stretching bands like (100)-(100) peaking at $\lambda=329.18$ and $329.03 \mathrm{~nm}$ or $(001)-(001)$. Here, the spectroscopic parameters were extracted from infrared [76] and laser magnetic resonance spectra $[77,78]$ of the $v_{3}$ and $v_{2}+v_{3}$ bands. In fact, according to the vibrational population distribution shown in Fig. 4 of Ref. [68], the population at high temperature is spread over many more levels such as those shown in Fig. 4. Clearly, the simulation of these additional vibrational bands improved the quality of the calculated high-temperature spectrum over a wide range of wavelengths as becomes obvious from the red trace in Fig. 7. Note that some modifications in the calculation of the different electronic transition moments (in particular the one of the band origin) were applied in the 2013 model as described in Ref. [68]. As a result, the contribution from the (000) - (000) band compared to the (010) - (010) bands decreased (see inset of Fig. 7). Also note the improved fitting of the two small features around $329.03 \mathrm{~nm}$, which is due to the (100) - (100) band. The assignment of the transitions is detailed in Fig. 3 of Ref. [68] and in Fig. 8.

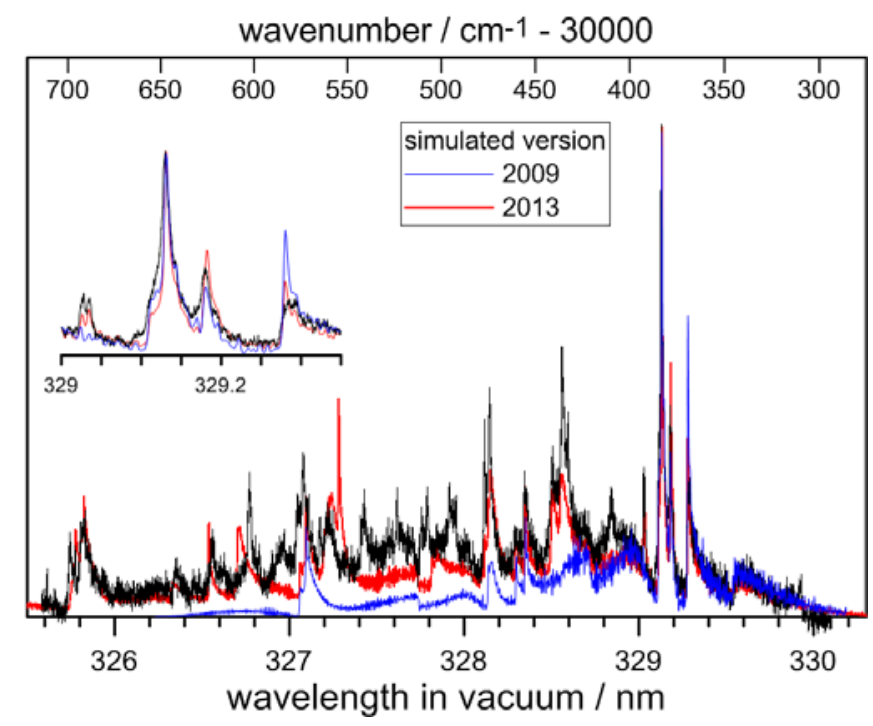

Fig. 7. Comparison of a near-linear LIF excitation spectrum of ${ }^{3} \mathrm{NCN}$ measured in a rich $\mathrm{C}_{2} \mathrm{H}_{2} / \mathrm{O}_{2} / \mathrm{N}_{2}$ low-pressure flame at $T=1660 \mathrm{~K}$ (black) with simulated spectra obtained on the basis of data from Ref. [42] (blue) and [68] (red); the inset shows a blow-up taken around the most intense band head (adapted from Lamoureux et al. [68]).

Quantitative detection of NCN in flames and shock tube experiments focused on the characteristic spectral features centered at $329 \mathrm{~nm}$. Fig. 8 zooms into this spectral range and compares the experimental spectra around the band origin obtained by LIF and CRDS in a low-pressure flame at $1660 \mathrm{~K}$ (described 
in more detail in Section 7.1). Simulated spectra calculated at $T=1660 \mathrm{~K}$ and $T=300 \mathrm{~K}$ are also shown. Interestingly, at high temperature, the characteristic feature of the cold spectra centered at $\lambda=$ $329.11 \mathrm{~nm}$, namely the $\tilde{\mathrm{A}}^{3} \Pi_{1}(000)-\tilde{\mathrm{X}}^{3} \Sigma_{\mathrm{g}}^{-}(000)$ band, is found much less intense than the component $\tilde{\mathrm{A}}^{3} \Sigma_{\mathrm{g}}^{+}-\tilde{\mathrm{X}}^{3} \Pi_{\mathrm{u}}$ of the $(010)-(010)$ hot band at $\lambda=329.13 \mathrm{~nm}$. The strongest peak corresponds to the $Q_{1}$ branch of this (010) - (010) hot band with rotational quantum numbers comprised between 25 and 60. At $300 \mathrm{~K}$, this feature could not be clearly analyzed by Herzberg and Travis [2] due the proximity of the intense band head of the $(000)-(000)$ transition. Moreover, because of the proximity of these two features (separated by $0.03 \mathrm{~nm}$ only), the first measurements of NCN at high temperature were erroneously attributed to the $(000)-(000)$ band $[31,38,41]$. Similarly, the line strength (attributed falsely to NCN (000) - (000)) was overestimated by a factor of 4 in Refs. [38,41]. Actually, NCN detection at high temperatures turns out to be very sensitive due to the appearance of this dominant hot band. This was clearly worked out by absorption measurements performed at temperatures of $298 \mathrm{~K}$, 850 K, and $1600 \mathrm{~K}$ by Dammeier and Friedrichs [72] (see Figs. 1 and 3 in Ref. [72]).

Another important aspect that needs to be considered for flame detection of $\mathrm{NCN}$ is the spectral shape of the dispersed fluorescence spectrum emitted after NCN excitation. LIF excitation spectra are typically recorded by collecting the fluorescence photons within a certain spectral bandwidth determined by the experimental setup (interference filter or monochromator). However, the reliability of the measured relative intensities in an excitation spectrum critically depends on the collection of fluorescence photons over a sufficiently broad range of wavelengths. Indeed, in case of NCN the spectral range of the emission depends on the excitation wavelength as is illustrated in the dispersed fluorescence spectra shown in Fig. 9. Note that no off-diagonal fluorescence bands $\left(\Delta \mathrm{v}_{i} \neq 0\right)$ were observed, in agreement with Smith et al. [39]. In this way, the measurement of the radiative lifetimes allows a straightforward determination of the Einstein emission coefficients. Moreover, from Fig. 9, it becomes obvious that the dispersed fluorescence spectrum is shifted toward shorter wavelengths for $\mathrm{v}_{2}>2$ transitions. Fig. 9 also gives an impression on the bandwidth (around $6 \mathrm{~nm}$ ) necessary to ensure collection of the entire dispersed fluorescence when scanning the excitation laser wavelength from 325.5 to $330.2 \mathrm{~nm}$, as was done in Fig. 7. 


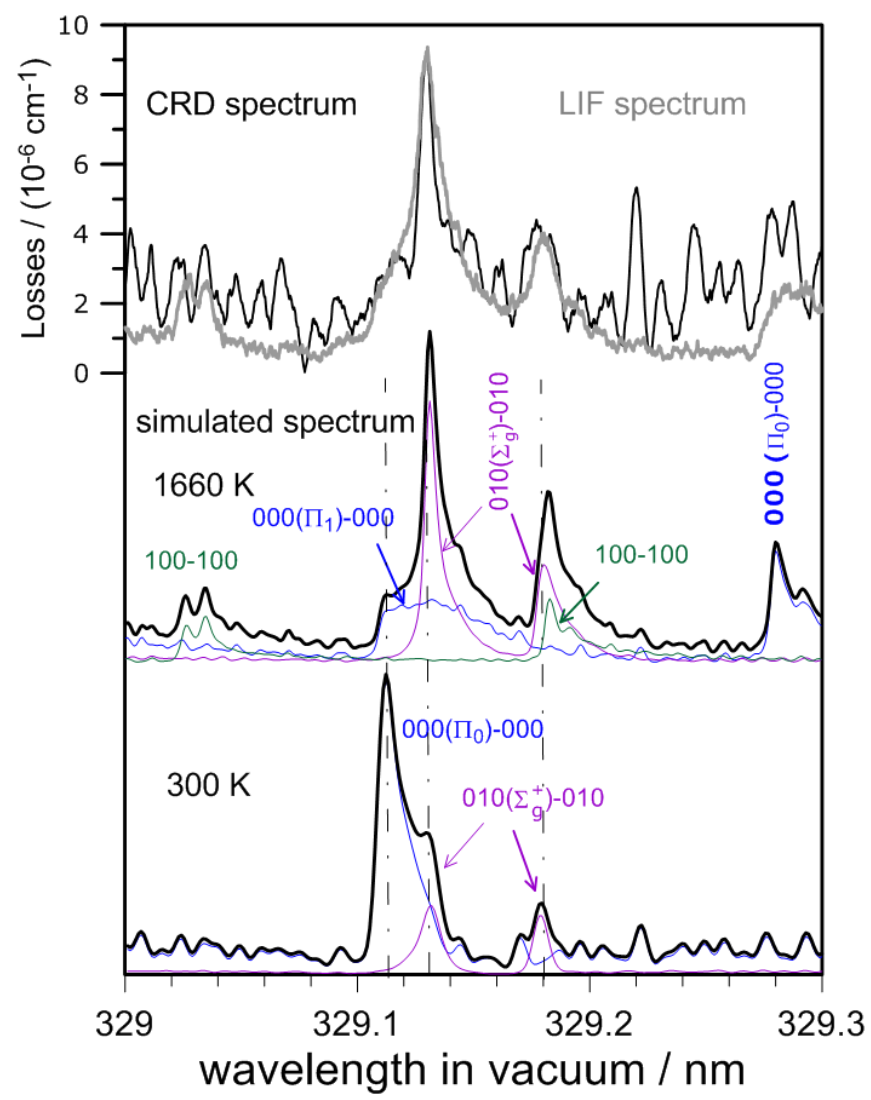

Fig. 8. ${ }^{3} \mathrm{NCN}$ spectra around the intense band head near $329.1 \mathrm{~nm}$; top panel: LIF excitation spectrum superimposed with the cavity ring-down loss spectrum both recorded in a rich low-pressure $\mathrm{C}_{2} \mathrm{H}_{2} / \mathrm{O}_{2} / \mathrm{N}_{2}$ flame at $T=1660 \mathrm{~K}$ (Ref. [68], see Section 7.1.2); middle $(T=1660 \mathrm{~K})$ and bottom $(T=300 \mathrm{~K})$ panels show spectra simulated with PGOPHER (convoluted Voigt lineshapes with $\Delta v_{\mathrm{G}}=0.3 \mathrm{~cm}^{-1}, \Delta v_{\mathrm{L}}=0.1 \mathrm{~cm}^{-1}$ ); colored lines highlight the contributions of the specified transitions to the overall absorption (black) (adapted from Lamoureux et al. [68]).

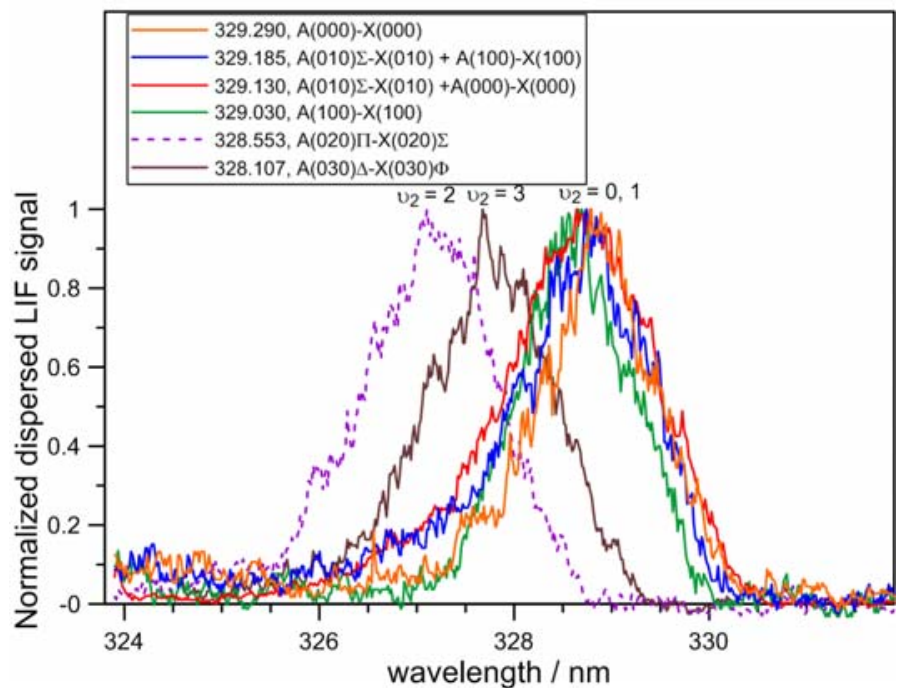

Fig. 9. Dispersed fluorescence spectra collected with a spectral bandwidth of $1.6 \mathrm{~nm}$, obtained after exciting different diagonal transitions of ${ }^{3} \mathrm{NCN}$ at high temperature $(1660 \mathrm{~K})$; the excitation wavelengths and the associated transitions are shown in the inset, the bending quantum numbers $v_{2}$ are additionally given near the maximum of each spectrum. 


\subsection{Determination of the absorption cross section of NCN}

Quantitative detection of NCN in flames and kinetics experiments has been almost exclusively performed for the triplet electronic ground state. Here, the knowledge of its (narrow-bandwidth) absorption cross section is crucial to relate absorbance to concentration. While the temperature and pressure dependence of absorption cross sections are straightforward to calculate for isolated rovibrational transitions [79], their determination in case of overlapped transitions is much facilitated by the use of simulation codes such as PGOPHER [74]. The capability of this simulation code to reproduce the extended LIF spectrum of NCN and to assign most of the additional bands, as was demonstrated in the preceding section, gives confidence also to use it for the calculation of cross sections and their extrapolation to different temperatures and pressures. Along these lines, the absorption cross section of the $\tilde{\mathrm{A}}-\tilde{\mathrm{X}}$ band of triplet NCN was calculated, on the one hand, from spectral simulations with the PGOPHER program [74] by Lamoureux et al. $[42,68]$ and, on the other hand, experimentally determined in direct absorbance measurements of samples with known NCN concentrations by Friedrichs and coworkers $[72,80]$.

\subsubsection{Lifetime measurements}

The calculation of the cross section was based on the knowledge of the electronic transition moments and the vibronic partition functions as described in Ref. [66]. The electronic transition moments can be directly calculated from the radiative lifetime. The inverse of the radiative lifetime, or the total radiative decay rate, can be determined from the extrapolation to zero-pressure in a Stern-Volmer plot where the fluorescence decay rate is plotted as a function of pressure. This implicitly supposes that the lifetime is not biased by non-radiative processes, and the inverse of the lifetime is thus equal to the Einstein emission coefficient. Smith et al. [39] measured the radiative lifetime of the most prominent features of the NCN LIF excitation spectrum with a nanosecond laser. The radiative lifetime at zero pressure was found to be equal to $183 \pm 6 \mathrm{~ns}(2 \sigma)$ with $4 \mathrm{~ns}$ average deviation and $2 \mathrm{~ns}$ precision for various $(000)-(000)$ and $(010)-(010)$ bands.

To check the reliability of this single reported key value, for this review the zero-pressure fluorescence lifetime of NCN was re-assessed in a joint effort of the Kiel (G.F.) and Lille (N.L, P.D.) groups. In this study, triplet $\mathrm{NCN}$ radicals were produced from photolysis of $\mathrm{NCN}_{3}$ by using an excimer laser operating at a wavelength of $193 \mathrm{~nm}$ as previously indicated in Ref. [72] and further outlined in Section 4. Following a time delay of $800 \mu$ s to allow for relaxation of the initially formed ${ }^{1} \mathrm{NCN}$ (see Section 4.2), the triplet NCN radicals were excited by a picosecond laser pulse at $329.1 \mathrm{~nm}$ (spectral bandwidth $<12 \mathrm{~cm}^{-1}$ ). The two collinear laser beams propagated through a low-pressure cell with $\mathrm{Ar}$ as bath gas. The time-resolved fluorescence decay was measured with a photomultiplier tube (PMT) after passing a monochromator (Acton SP2300i, grating $1200 \mathrm{~g} / \mathrm{mm}$ ) with a spectral bandwidth of $328 \pm 3$ 
$\mathrm{nm}$. The signal was sampled by an oscilloscope with 12 bit vertical resolution (LeCroy HDO4000, 1 $\mathrm{GHz}$ ). The fluorescence signals were averaged over 500 laser shots, and at least four such measurements were performed at each pressure. From Stern-Volmer plots (see Fig. S1 of the Supplementary Material), values of $182.2 \mathrm{~ns}$ and $184.4 \mathrm{~ns}$ were obtained for the radiative lifetime of $\mathrm{NCN}$ at two different mole fractions of the precursor $\mathrm{NCN}_{3}$. The relative standard deviations of the fluorescence lifetimes were below $1 \%$. The resulting mean value is $183.3 \pm 7 \mathrm{~ns}$ where the uncertainty corresponds to two standard deviations. It is in agreement with the value of $183 \pm 6$ ns previously reported by Smith et al. [39]. Note that their investigation has been performed at rather different experimental conditions regarding both $\mathrm{NCN}$ production (microwave-discharge of $\mathrm{CF}_{4}$ in $\mathrm{N}_{2}$ vs. laser photolysis of $\mathrm{NCN}_{3}$ ) and pulse duration of the excitation laser (ns vs ps). Taking this into account, a value of $183 \pm 7 \mathrm{~ns}$ can be considered reliable and is recommended.

Using this value, the absorption cross section was calculated with PGOPHER. Note that the program calculates the partition functions exclusively for electronic ground state levels included in the simulation (i.e. the levels shown in Fig. 4). Hence, the calculated absorption cross section value has been corrected by the ratio between the vibronic partition functions of all the levels explicitly described in the simulation and the total partition functions summed over all the vibronic levels. As expected, the obtained Doppler-limited absorption cross section was found to have a negative temperature dependence (red line in Fig. 10), which can be approximated over the temperature range from 750 to $2500 \mathrm{~K}$ with an average uncertainty of $\pm 22 \%$ :

$$
\sigma\left({ }^{3} \mathrm{NCN}, 329.13 \mathrm{~nm}\right)(T) / \mathrm{cm}^{2}=3.30 \times 10^{-15} \exp \left(-1.80 \times 10^{-3} \times T / \mathrm{K}\right)
$$

The error estimate takes into account the $\pm 3.8 \%$ uncertainty of the radiative lifetime and $\pm 20 \%$ coming from the overall simulation, including the calculation of the vibronic partition function [68].

\subsubsection{Direct absorption measurements}

Dammeier and Friedrichs [72] reported a direct measurement of the high-temperature absorption cross section of $\mathrm{NCN}$ in shock tube experiments on $\mathrm{NCN}_{3}$. By assuming quantitative formation of $\mathrm{NCN}$ from $\mathrm{NCN}_{3}$ (see Section 4.2 below), the absorption cross section of triplet $\mathrm{NCN}$ was determined from the measured absorption signals observed after shock-heating of highly diluted $\mathrm{NCN}_{3} / \mathrm{Ar}$ mixtures containing $<15 \mathrm{ppm}$ of $\mathrm{NCN}_{3}$. Time-resolved measurements were performed by using difference amplification laser absorption spectroscopy with a tunable narrow-bandwidth $(<10 \mathrm{MHz})$, frequencydoubled UV ring-dye laser at wavelengths around $\lambda=329.1 \mathrm{~nm}$ (overall wavelength uncertainty $\Delta \lambda=$ $\pm 1.6 \times 10^{-4} \mathrm{~nm}$ ). The temperature and pressure range of the experiments was $750-2250 \mathrm{~K}$ and $20-250$ $\mathrm{kPa}$, respectively. No significant pressure dependence of the absorption cross section was observed. This is consistent with PGOPHER simulations: Assuming a typical pressure-broadening coefficient of $0.6 \mathrm{GHz}$ $(T=1600 \mathrm{~K}, p=100 \mathrm{kPa}$ ) for a three-atomic species [81] results in a merely $6 \%$ lower peak absorption 
cross section compared to a Doppler-limited case when changing the pressure from 0.05 to $0.1 \mathrm{MPa}$. The reported Doppler-limited absorption cross section data are shown in Fig. 10 and are a factor of about 2.5 lower than those determined by Lamoureux and coworkers [68] from the radiative lifetime measurements and spectral simulations as explained in the preceding Section. This unexpectedly large discrepancy has been not resolved until very recently. Faßheber et al. [80] re-investigated the absorption cross section using two independent detection systems and performed a detailed analysis of potential error sources including a careful analysis of the stability and chemical composition of the reactant gas mixtures. It turned out that the used gas mixtures contained significant amounts of previously unidentified molecular nitrogen (see also Section 4.1). Moreover, decomposition and wall losses of $\mathrm{NCN}_{3}$ in the storage flasks and the gas supply lines showed that a correction by at least a factor of 1.5 needs to be applied to the earlier data from Dammeier and Friedrichs (indicated by the green arrow in Fig. 10). The newly measured data points from Faßheber et al. [80] are included in Fig. 10 as open circles. The absolute values and temperature dependence are in agreement with the calculations of Lamoureux et al. [68] within error limits. Finally, the result of the shock tube study by Vasudevan et al. [31] as detailed in Section 6.1.1 are included in Fig. 10 as a blue line. Within the reported uncertainty of a factor of two, which is mostly related to the modeled $\mathrm{CH}$ concentration profiles of the shock-heated ethane $/ \mathrm{He} / \mathrm{N}_{2}$ gas mixtures, these data are in reasonable agreement with the data from Ref. [68] and [80] as well.

In contrast to ${ }^{3} \mathrm{NCN}$, quantitative detection of ${ }^{1} \mathrm{NCN}$ has only been reported so far in one kinetic study by Dammeier et al. [82]. Here, ${ }^{1} \mathrm{NCN}$ has been detected as a product of the thermal decomposition of $\mathrm{NCN}_{3}$ behind shock waves by using narrow-bandwidth laser absorption spectroscopy. Part of the band head of the $\widetilde{c}^{1} \Pi_{u}(000) \leftarrow \widetilde{a}^{1} \Delta_{g}(000)$ transition has been measured at a temperature of about $740 \mathrm{~K}$ and a pressure of $39 \mathrm{kPa}$, revealing a structureless spectral feature near $332.8 \mathrm{~nm}$. By simultaneous fits of the kinetic profiles for ${ }^{3} \mathrm{NCN}$ and ${ }^{1} \mathrm{NCN}$ (see Fig. 12 in Section 4.1), the temperature dependence of the mainly Doppler-limited absorption cross section has been estimated for the temperature range $618 \mathrm{~K}<T<1231 \mathrm{~K}$. It can be expressed in the form $\sigma\left({ }^{1} \mathrm{NCN}, 332.8290 \mathrm{~nm}\right)(T) / \mathrm{cm}^{2}$ $=1.66 \times 10^{-16}-1.05 \times 10^{-19} \times T / \mathrm{K}$ with an uncertainty of $\pm 50 \%$. It turns out that the absolute values are similar to those for ${ }^{3} \mathrm{NCN}$. Note, however, that the results depend on the assumed mechanistic model for ${ }^{1} \mathrm{NCN}$ formation and relaxation (see Section 5.2) and does not yet account for the potential extra uncertainties related to the assumed initial $\mathrm{NCN}_{3}$ concentration mentioned above. 


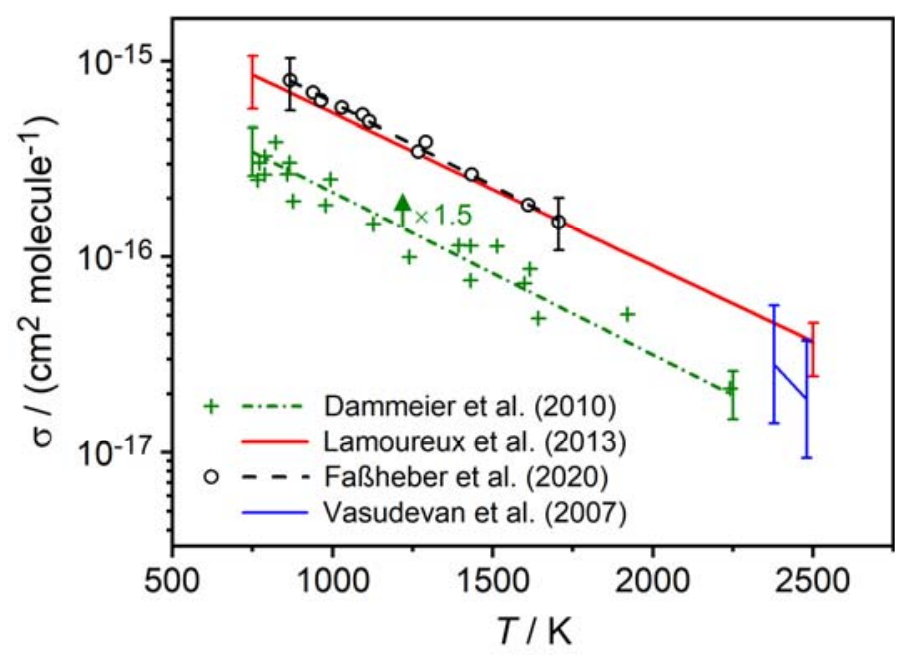

Fig. 10. Doppler-limited absorption cross section of ${ }^{3} \mathrm{NCN}$ at $\lambda=329.1302 \mathrm{~nm}$ as a function of temperature. Comparison of results from theoretical calculations using PGOPHER, Lamoureux et al. (2013) [68], and direct absorption measurements using either the reaction $\mathrm{CH}+\mathrm{N}_{2}$, Vasudevan et al. (2007) [31], or the thermal decomposition of $\mathrm{NCN}_{3}$ as NCN radical source, Dammeier et al. (2010) [70] and Faßheber et al. 2020 [78]. The arrows indicate a minimal necessary correction of the data from Ref. [72] as outlined in Ref. [80] (see text); adapted from Faßheber et al. [80].

\section{Thermochemistry of NCN}

As already mentioned in the Introduction, the knowledge of the thermochemistry of NCN is crucial because it directly affects the branching ratio of the reaction $\mathrm{NCN}+\mathrm{H}$, and subsequently the prompt-NO switch.

\subsection{Vibrational energy levels and rotational constant}

From the simulated spectrum, it is also possible to derive the vibrational wavenumbers and rotational constant of the NCN free radical. These quantities are closely related, via the corresponding partition functions, to thermodynamic data like heat capacities, standard entropies, and thermal corrections for energies (see e.g. Refs. $[83,84]$ ). Experimental results reported in the literature are presented in Table 1. Most of these values were obtained from spectroscopic band analyses and represent fundamental vibrational wavenumbers. The rotational constant $B_{0}$ is the value for the ground vibrational state of NCN.

Nowadays, molecular properties can also be predicted by quantum chemical methods, often with sufficient accuracy [85]. Selected results of such calculations for NCN vibrational wavenumbers and the rotational constant are listed in Table 2. Here, in contrast to the experimental results (Table 1), mostly harmonic vibrational wavenumbers have been published. Fundamental vibrational wavenumbers 
can be calculated from perturbation theory (see e.g. Ref. [86]) but are less frequently reported; they are marked in Table 2. The rotational constants $B_{\mathrm{e}}$ in Table 2 are those calculated from equilibrium geometries. For the relation between $B_{\mathrm{e}}$ and $B_{0}$ see e.g. Refs. [83,84,87].

\section{Table 1}

Experimentally derived vibrational wavenumbers for ${ }^{3} \mathrm{NCN}$, rounded to integer values $\left(\omega_{1}\right.$ : symmetric stretch, $\omega_{2}$ : bending, $\omega_{3}$ : antisymmetric stretch) and rotational constants $\left(B_{0}\right)\left(\right.$ units: $\left.\mathrm{cm}^{-1}\right)$.

\begin{tabular}{|l|l|l|l|l|l|}
\hline$\omega_{1}$ & $\omega_{2}$ & $\omega_{3}$ & $B_{0}$ & Method & Ref. \\
\hline & $370 \pm 50$ & & 0.3968 & gas, UV absorption & {$[2]$} \\
\hline 1197 & 423 & 1475 & & Ar matrix, IR absorption & {$[3]$} \\
\hline & 395 & & & Ar matrix, IR absorption & {$[88]$} \\
\hline & & 1467 & 0.3972 & gas, IR absorption & {$[76]$} \\
\hline 1224 & 429 & & 0.3973 & gas, UV absorption & {$[69]$} \\
\hline 1245 & 423 & & & gas, UV absorption & {$[70]$} \\
\hline
\end{tabular}

a according to Ref. [69]

\section{Table 2}

Calculated harmonic vibrational wavenumbers for ${ }^{3} \mathrm{NCN}$, rounded to integer values $\left(\omega_{1}\right.$ : symmetric stretch, $\omega_{2}$ : bending, $\omega_{3}$ : asymmetric stretch) and rotational constants $\left(B_{\mathrm{e}}\right)$ for equilibrium bond lengths (units: $\mathrm{cm}^{-1}$ ).

\begin{tabular}{|c|c|c|c|c|c|}
\hline$\omega_{1}$ & $\omega_{2}$ & $\omega_{3}$ & $B_{\mathrm{e}}$ & Method & Ref. \\
\hline 1217 & 406 & 1334 & 0.3852 & $\mathrm{CASSCF} / \mathrm{pVDZ}$ & [89] \\
\hline 1234 & 357 & 1328 & 0.3957 & $\operatorname{CCSD}(\mathrm{T}) / \mathrm{TZ} 2 \mathrm{P}$ & [89] \\
\hline 1221 & 417 & 1329 & 0.3912 & $\mathrm{CASSCF} / 6 s 4 p 2 d 2 f$ & [90] \\
\hline 1228 & 410 & 1516 & 0.3953 & B3LYP/6-31G(d) & [91] \\
\hline 1253 & 431 & 1453 & 0.3972 & UU-CCSD(T)/cc-pVQZ & [92] \\
\hline 1292 & 460 & 1425 & & B2PLYPD3/cc-pVTZ & [17] \\
\hline $1265^{\mathrm{a}}$ & $459^{\mathrm{a}}$ & $1430^{\mathrm{a}}$ & & VPT2/B2PLYPD3/cc-pVTZ & [17] \\
\hline $1218^{a}$ & $423^{\mathrm{a}}$ & $1486^{\mathrm{a}}$ & & UU-CCSD(T)/CBS(TZ;QZ) (+ corrections) & [17] \\
\hline 1260 & 434 & 1459 & 0.3993 & ae-CCSD(T)/cc-pwCVQZ & [37] \\
\hline $1240^{\mathrm{a}}$ & $433^{\mathrm{a}}$ & $1444^{\mathrm{a}}$ & $0.3980^{\mathrm{b}}$ & VPT2/ae-CCSD(T)/cc-pwCVQZ & [37] \\
\hline
\end{tabular}

${ }^{a}$ Fundamental vibrational wavenumbers obtained on the basis of second-order vibrational perturbation theory (VPT2).

b $B_{0}$.

It turns out that for $\mathrm{NCN}$ the differences between harmonic and fundamental wavenumbers are small, resulting in differences of the corresponding vibrational zero-point energies on the order of 
merely a few hundredth $\mathrm{kJ} \mathrm{mol}^{-1}$ [37]. A peculiarity of NCN noted in Ref. [17] appears to be the sensitivity of the calculated wavenumber of the asymmetric stretching vibration ( $\omega_{3}$ in Table 2$)$ to spin restriction at the Hartree-Fock and/or the $\operatorname{CCSD}(\mathrm{T})$ level of theory. Here, deviations by more than 1000 $\mathrm{cm}^{-1}$ compared to the experimental value were obtained in some cases, leading to errors in the predicted vibrational zero-point energy of up to $12 \mathrm{~kJ} \mathrm{~mol}^{-1}$. The problem, which also concerns the CBS limit of the $\operatorname{CSD}(\mathrm{T})$ energy, can be overcome by using unrestricted wave functions. Only values unbiased by this artifact are collected in Table 2; for details see Ref. [17]. An overview of NCN vibrational energy levels of the electronic ground state and several electronically excited states that were published prior to the year 2000 is given in Ref. [93].

\subsection{Molar heat capacity}

The heat capacity of an ideal gas, $C_{p}{ }^{\circ}$, can be related to the corresponding spectroscopic properties via the molecular partition function $Q$. One has: $C_{p}{ }^{\circ} / R=T^{2}\left(\partial^{2} \ln Q / \partial T^{2}\right)+2 T(\partial \ln Q / \partial T)+1$ with $R$ denoting the gas constant (see e.g. Refs. $[83,84]$ ). The partition function in turn can be calculated at different levels of approximation (see e.g. Refs. $[83,84]$ ). For small molecules without torsional degrees of freedom like NCN, a rigid rotor harmonic oscillator (RRHO) approach is often sufficiently accurate for typical combustion conditions. In the following, we compare heat capacities calculated in RRHO approximation on the basis of molecular data from different sources. The calculations were performed with the program code PAC99 from McBride and Gordon [94]; standard state is the ideal gas at $p=0.1$ $\mathrm{MPa}$. The results of these calculations are represented in the widely used seven-coefficient NASA polynomials (see e.g. Refs. [95,96]): $C_{p}{ }^{\circ}(T) / R=a_{1}+a_{2} T+a_{3} T^{2}+a_{4} T^{3}+a_{5} T^{4}$, with the temperature $T$ in K. For the molar enthalpy $H^{\circ}$ and entropy $S^{\circ}$, it follows: $H^{\circ}(T) / R T=a_{1}+a_{2} T / 2+a_{3} T^{2} / 3+a_{4} T^{3} / 4+$ $a_{5} T^{4} / 5+a_{6} / T$ and $S^{\circ}(T) / R=a_{1} \ln T+a_{2} T+a_{3} T^{2} / 2+a_{4} T^{3} / 3+a_{5} T^{4} / 4+a_{7}$.

We note that coefficients of the NASA polynomials are usually given/stored in machine readable format [95,97], where the first seven entries are the coefficients $a_{1}$ through $a_{7}$ for $T=1000-6000 \mathrm{~K}$ and the second seven entries the same for $T=200-1000 \mathrm{~K}$ (see Table S1 of the Supplementary Material). Note that also a less common nine-coefficient format exists [97,98] (see Table S2). 


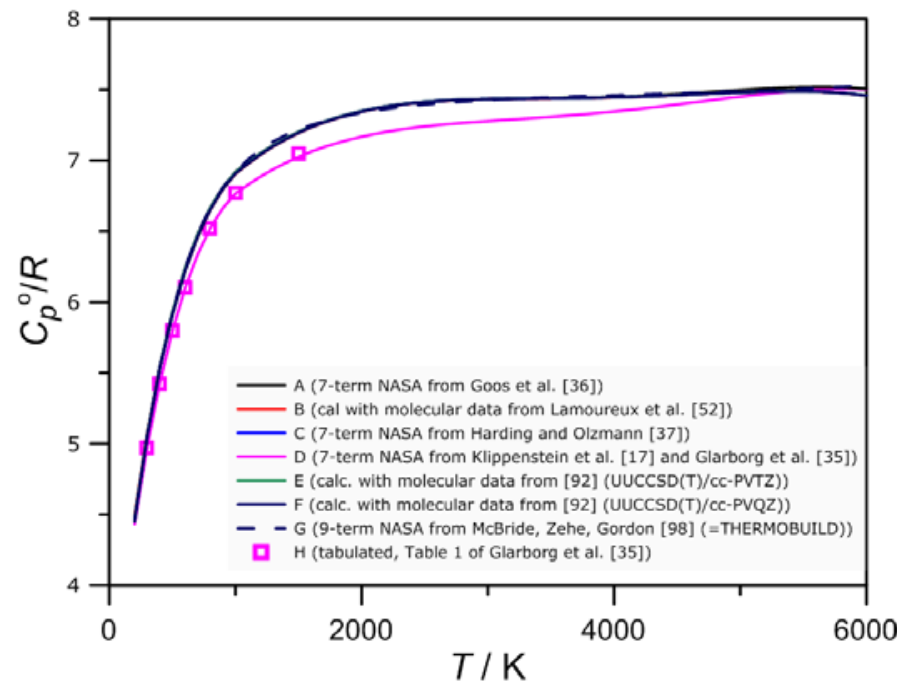

Fig. 11. Plot of the reduced ${ }^{3} \mathrm{NCN}$ heat capacities $C_{p}{ }^{\circ} / R$ as a function of $T$ from different sources. Except for the highest temperatures, the curves from sources $\mathrm{A}$ to $\mathrm{C}$ and $\mathrm{E}$ to $\mathrm{G}$ are very close to each other (for relative deviations see Fig. S2 of the Supplementary Material). A: Goos et al. [36], B: Lamoureux et al. [52], C: Harding and Olzmann [37], D: Klippenstein et al. [17] and Glarborg et al. [35], E and F: calculated with data from Klippenstein et al. [92], G: McBride et al. [98], H: Glarborg et al. [35].

A comparison of heat capacities as a function of temperature from different sources is made in Fig. 11. These sources/models are denoted by capital letters $A$ to $H$ and are briefly characterized in the inset of Fig. 11; more details are given in Table S1 to S3 of the Supplementary Material. From Fig. 11 it is obvious that the values from almost all sources fall into a very narrow range. Relative deviations are plotted in Fig. S2 of the Supplementary Material. For models A to C and E to G, these deviations are well below $1 \%$ over the entire temperature range $200 \mathrm{~K}<T<6000 \mathrm{~K}$.

The very similar data sets $\mathrm{D}$ and $\mathrm{H}$ show larger deviations from the other models. We note that the data points from $\mathrm{H}$ and the curve from $\mathrm{D}$ stem from the same publication [35] and were calculated probably relying on the same molecular data set, which is, however, not readily available. Our recalculation with the most recent available molecular data set from one of the authors (Klippenstein et al. [92]) gave heat capacities (curves E and F) in very good agreement with the data from A, B, C, and G. We were not able, however, to reproduce the heat capacities from D and H. So, the reason for the deviation remains unclear, and there may be assumptions/approximations not documented along with the published data of D and H in Refs. [17,35]. Note that such an appreciable deviation of the heat capacity parametrization causes significant uncertainties, for example about $3 \mathrm{~kJ} \mathrm{~mol}^{-1}$ for the enthalpy of formation at $1500 \mathrm{~K}$. Such deviations may result in subsequent uncertainties for the NCN and NO predictions in flames. Goos et al. [36] reported a decrease of the NCN peak mole fraction by a factor 0.75 with the increase of the enthalpy of formation by $12 \mathrm{~kJ} \mathrm{~mol}^{-1}$, see Figs. 1 and 3 in Ref. [36].

In view of the rather small differences in the heat capacities calculated with the seven-coefficients NASA polynomials A [36] and C [37], we recommend these data sets for the calculation of thermal 
corrections to enthalpies and energies. With the data from Ref. [37], one obtains an enthalpy correction of $H^{\circ}{ }_{298.15}-H^{\circ}{ }_{0}=10.191 \mathrm{~kJ} \mathrm{~mol}^{-1}$, and the data from Ref. [36] give $10.180 \mathrm{~kJ} \mathrm{~mol}^{-1}$. The small difference of $0.011 \mathrm{~kJ} \mathrm{~mol}^{-1}$ is negligible for almost all practical applications.

Note that we also report seven-coefficients NASA polynomials for ${ }^{1} \mathrm{NCN}$ in Table S4 of the Supplemental Material.

\subsection{Enthalpy of formation}

The enthalpy of formation of a species is a most influential quantity not only for thermodynamic but also for kinetic considerations because it is intrinsically connected with the energies of activation of the forward and backward step of a reversible reaction (see e.g. Ref. [99]). Within a very complex reaction network like hydrocarbon combustion, complicated dependences of reaction pathways from energetic quantities of participating species can occur. Therefore, any adequate description and analysis of the role of $\mathrm{NCN}$ in the formation of prompt-NO requires reliable values for the enthalpy of formation, $\Delta_{\mathrm{f}} H^{\circ}(\mathrm{NCN})$, as a function of temperature.

Since NCN is a reactive radical species, experimental determinations of $\Delta_{\mathrm{f}} H^{\circ}(\mathrm{NCN})$ are difficult and so far rather indirect and scarce. Accordingly, most of the data on $\Delta_{\mathrm{f}} H^{\circ}(\mathrm{NCN})$ published so far were obtained from quantum chemical calculations for the triplet species. Extensive discussions of $\Delta_{\mathrm{f}} H^{\circ}\left({ }^{3} \mathrm{NCN}\right)$ values obtained with different experimentally-based and theoretical methods, including thermochemical, networks are given in Refs. [17,35-37] and are, therefore, not repeated here. In Table 3, selected values that represent the different approaches are listed. With exception of the ATcT values collected at the end of the table, the entries are grossly in chronological order. Considering the very good agreement between the most recent quantum chemical results, all obtained with compound methods (ANL0, ANL1, HEAT456-QPH) that were specifically designed for the calculation of thermochemical quantities, and the result of the most recent ATcT analysis (version 122p), one can recommend a value in the range $\Delta_{\mathrm{f}} H^{\circ}{ }_{0}\left({ }^{3} \mathrm{NCN}\right)=451 \pm 1 \mathrm{~kJ} \mathrm{~mol}^{-1}$ as being most likely. Note that with the enthalpy differences $H^{\circ}{ }_{298.15}\left({ }^{3} \mathrm{NCN}\right)-H^{\circ}{ }_{0}\left({ }^{3} \mathrm{NCN}\right)=10.191 \mathrm{~kJ} \mathrm{~mol}^{-1}([37]$, see above $), H^{\circ}{ }_{298.15}\left(\mathrm{C}_{\text {graphite }}\right)-H^{\circ}{ }_{0}\left(\mathrm{C}_{\text {graphite }}\right)=1.051$ $\mathrm{kJ} \mathrm{mol}^{-1}$ [100], and $H^{\circ}{ }_{298.15}\left(\mathrm{~N}_{2}\right)-H^{\circ}{ }_{0}\left(\mathrm{~N}_{2}\right)=8.670 \mathrm{~kJ} \mathrm{~mol}^{-1}$ [100], one obtains for the temperature correction of the enthalpy of formation $\Delta_{\mathrm{f}} H^{\circ} 298.15\left({ }^{3} \mathrm{NCN}\right)-\Delta_{\mathrm{f}} H^{\circ}{ }_{0}\left({ }^{3} \mathrm{NCN}\right)=0.47 \mathrm{~kJ} \mathrm{~mol}^{-1}$. 
Table 3

Enthalpy of formation of ${ }^{3} \mathrm{NCN}$ at $T=0 \mathrm{~K}$ and $T=298.15 \mathrm{~K}$ (unit: $\mathrm{kJ} \mathrm{mol}{ }^{-1}$ ).

\begin{tabular}{|l|l|l|l|}
\hline$\Delta_{\mathrm{f}} H_{0}^{\circ}$ & $\Delta_{\mathrm{f}} H^{\circ}{ }_{298.15}$ & Method/data source & Ref. \\
\hline $472 \pm 21$ & $473 \pm 21$ & extended literature review (up to December 1970) & {$[100]$} \\
\hline & $452 \pm 17$ & experiment (NCN- photoelectron spectroscopy) & {$[101]$} \\
\hline $452.3 \pm 3$ & $452.8 \pm 3$ & theory (CBS-QCI/APNO) & {$[101]$} \\
\hline $466 \pm 3$ & & experiment (NCN photodissociation dynamics) & {$[66]$} \\
\hline & $448.7 \pm 3.4$ & theory (average from CBS-QB3, CBS-APNO, G3B3, G3, G4) & {$[91]$} \\
\hline $457.9 \mathrm{a}$ & & theory (isodesmic reaction, CCSD(T), CBS limit) & {$[16]$} \\
\hline & $457.8 \pm 2.0$ & literature review and active thermochemical tables analysis & {$[36]$} \\
\hline $450(<456)$ & thermochemical kinetic analysis of NCN + H reaction & {$[102]$} \\
\hline 450.44 & 454.52 & theory (W3X-L) & {$[103]$} \\
\hline 450.24 & $452.2 \pm 1.0$ & theory (HEAT456-QPH) & {$[37]$} \\
\hline $445.3 \pm 1.8$ & $445.7 \pm 1.8$ & ATcT (version 1.112) (2013) & {$[17]$} \\
\hline $455.9 \pm 1.3$ & $456.4 \pm 1.3$ & ATcT (version 1.118) (2015) & {$[106]$} \\
\hline $450.42 \pm 0.64$ & $450.83 \pm 0.64$ & ATcT (version 1.122p) (2020) & {$[104]$} \\
\hline
\end{tabular}

${ }^{\mathrm{a}}$ no temperature given by Teng et al. [16]

${ }^{\mathrm{b}}$ Active Thermochemical Tables (version of the thermochemical network) (year)

\section{NCN production for kinetics experiments}

Different experimental approaches have been used to produce NCN as a reactant for kinetics experiments. To the best of our knowledge, the first experimental determination of rate constants for NCN reactions was reported by Baren and Hershberger in 2002 [107]. Based on earlier spectroscopic studies [2], these workers used laser flash photolysis of diazomethane/dicyan $\left(\mathrm{CH}_{2} \mathrm{~N}_{2} / \mathrm{C}_{2} \mathrm{~N}_{2}\right)$ mixtures at wavelengths of $193 \mathrm{~nm}$ or $248 \mathrm{~nm}$ to produce $\mathrm{NCN}$ radicals, presumably via the reaction ${ }^{1} \mathrm{CH}_{2}+\mathrm{C}_{2} \mathrm{~N}_{2}$ $\rightarrow \mathrm{NCN}+\mathrm{C}_{2} \mathrm{H}_{2}$. Later on, in a number of experimental studies at temperatures below ca. $500 \mathrm{~K}$, laser photolysis of cyanogen azide $\left(\mathrm{NCN}_{3}\right)$ at wavelengths of $248 \mathrm{~nm}$ or $193 \mathrm{~nm}$ was employed as NCN radical source [82,102,108-110].

A first high-temperature rate constant determination of the reaction $\mathrm{NCN}+\mathrm{H}$ has been reported in 2007 by Vasudevan et al. [31]. These authors analyzed NCN concentration-time profiles observed in shock-heated ethane/nitrogen/helium/argon gas mixtures. The use of $\mathrm{NCN}_{3}$ as a direct thermal precursor 
of NCN in high-temperature shock tube studies has been described since about the year 2010 by the groups of Friedrichs $[72,80,82,102,111-114]$ and Olzmann $[115,116]$.

Since the photolysis or thermal decomposition of $\mathrm{NCN}_{3}$ currently appears to be the most widely used source of $\mathrm{NCN}$ in kinetics experiments, we outline in the following important aspects of this approach, touching both practical and more fundamental questions.

\section{1 $\mathrm{NCN}_{3}$ as a precursor of $\mathrm{NCN}$}

The pyrolysis of gaseous cyanogen azide, $\mathrm{NCN}_{3}$, has been first discussed as an efficient $\mathrm{NCN}$ source in the work of Marsh and Hermes [117]. Shortly after, in a flash photolysis study of gaseous $\mathrm{NCN}_{3}$, Kroto [5] observed strong absorption features that he assigned to NCN: ${ }^{1} \mathrm{NCN}\left(\widetilde{\mathrm{c}}^{1} \Pi_{\mathrm{u}} \leftarrow \widetilde{\mathrm{a}}^{1} \Delta_{\mathrm{g}}\right)$ and ${ }^{3} \mathrm{NCN}$ $\left(\tilde{\mathrm{A}} \Pi_{\mathrm{u}} \leftarrow \tilde{\mathrm{X}}^{3} \Sigma_{\mathrm{g}}^{-}\right.$), where the singlet NCN was mentioned to be likely formed first. $\mathrm{NCN}_{3}$ is a highly explosive and presumably very toxic substance, hence great care should be taken during synthesis and handling of this compound. Marsh [118] describes the pure azide "as a colorless oil which detonates with great violence when subject to mild mechanical, thermal, or electrical shock", therefore gaseous $\mathrm{NCN}_{3}$ should never be condensed. Typically, $\mathrm{NCN}_{3}$ is handled as a solution in aprotic solvents [119] or kept in the gas phase at partial pressures of a few $\mathrm{hPa}$, preferentially diluted in inert gases. Nevertheless, the authors of this paper experienced occasional deflagrations during $\mathrm{NCN}_{3}$ synthesis of gaseous samples. Purification steps such as freeze-pump cycles are not possible. Instead, $\mathrm{NCN}_{3}$ is directly synthesized in high purity by using variants [72,116] of a method described by Milligan et al. [88]. In this approach, evaporated gaseous cyanogen bromide, $\mathrm{BrCN}$, quantitatively reacts with excess solid sodium azide, $\mathrm{NaN}_{3}$, at room temperature according to the reaction $\mathrm{NaN}_{3}+\mathrm{BrCN} \rightarrow \mathrm{NCN}_{3}+\mathrm{NaBr}$. The total reaction time, typically many hours, is chosen long enough to make sure a complete conversion of $\mathrm{BrCN}$ but short enough to minimize the slow decomposition of the $\mathrm{NCN}_{3}$ product. Decomposition of pure and diluted $\mathrm{NCN}_{3}$ gas mixtures has been reported to be on the order of $5-15 \%$ per day and yields a solid substance at the walls of the reaction flask $[72,80]$. Purity of the freshly synthesized products has been analyzed by mass spectrometry and FTIR spectroscopy. The reported main impurity is non-reacted $\mathrm{BrCN}$, which is typically present on the percent mole fraction level $[72,80,120]$. At longer storage or reaction times, significant amounts of cyanogen, $(\mathrm{CN})_{2}$, are discernible in the FTIR spectra; freshly synthesized gas mixtures contained about $1 \%$ [80]. Stored mixtures also show increasing mass peaks at $m / z=54\left(\mathrm{CN}_{3}\right.$, e.g. $\left.\mathrm{NC}-\mathrm{NN}^{+}\right)$and $80\left(\mathrm{C}_{2} \mathrm{~N}_{4}\right.$, e.g. $\left.\mathrm{NC}-\mathrm{NN}-\mathrm{CN}^{+}\right)$that have been tentatively assigned to fragments of the $\mathrm{NCN}_{3}$ dimerization/polymerization product or the $\mathrm{NCN}$ dimer azodicarbonitrile $(\mathrm{NCN}=\mathrm{NCN})[80,121]$. Note that the latter has been described as the main product of $\mathrm{NCN}_{3}$ pyrolysis at $200{ }^{\circ} \mathrm{C}$ as well [117]. Very recently, molecular nitrogen (but not $\mathrm{O}_{2}$, as may be expected in case of leaks) has been identified as another significant and difficult to quantify impurity. Not unexpected, it is presumably formed as direct product of $\mathrm{NCN}_{3}$ decomposition and polymerization. Analyzed by means 
of quantitative gas chromatography, freshly prepared $\mathrm{NCN}_{3}$ mixtures contained $15-25 \% \mathrm{~N}_{2}$ after a synthesis time of 10 hours [80].

Despite these difficulties in using $\mathrm{NCN}_{3}$ as a quantitative source of $\mathrm{NCN}$ radicals, $\mathrm{NCN}_{3}$ can be still considered first choice for reaction kinetics studies. $\mathrm{NCN}_{3}$ storage mixtures should be used for a maximum of a few days to avoid interfering impurity-related chemistry. Impurities of $\mathrm{BrCN}$, however, due to its high thermal stability, are less problematic in shock tube studies where even addition of up to $50 \% \mathrm{BrCN}$ did not change the observed NCN concentration-time profiles [72]. Moreover, studies of bimolecular reactions $\mathrm{NCN}+\mathrm{X}$ have been performed under pseudo-first order conditions with $\mathrm{X}$ acting as the excess component in most cases. Under such conditions, accurate knowledge of the absolute $\mathrm{NCN}_{3}$ and with it $\mathrm{NCN}$ concentration is less important for a reliable rate constant determination.

\subsection{NCN formation in the pyrolysis and UV photolysis of $\mathrm{NCN}_{3}$}

Both thermal decomposition and UV photolysis of cyanogen azide is known to yield NCN in its first electronically excited singlet state, $\widetilde{a}^{1} \Delta_{\mathrm{g}}$ (in short ${ }^{1} \mathrm{NCN}$ ) instead of its ground state, $\tilde{\mathrm{X}}^{3} \Sigma_{\mathrm{g}}^{-}$(in short $\left.{ }^{3} \mathrm{NCN}\right)$.

The ground state potential energy surface of $\mathrm{NCN}_{3}\left({ }^{1} \mathrm{~A}^{\prime}, S_{0}\right)$ has been first calculated by Benard et al. [122] at density functional B3LYP level of theory. Endothermic dissociation to the spin-correlated products ${ }^{1} \mathrm{NCN}+\mathrm{N}_{2}\left(\tilde{\mathrm{X}}^{1} \Sigma_{\mathrm{g}}^{+}\right)$was found to exhibit a small additional barrier along the dissociation coordinate. Starting from the ${ }^{3} \mathrm{NCN}+\mathrm{N}_{2}\left(\tilde{\mathrm{X}}^{1} \Sigma_{\mathrm{g}}^{+}\right)$products, the repulsive ${ }^{3} \mathrm{~A}^{\prime \prime}\left(T_{1}\right)$ surface was also constructed yielding a level crossing of the $S_{0}$ and $T_{1}$ states near the singlet surface maximum. In such a case, it is expected that ISC probabilities are small compared to the specific dissociation rate constants and therefore ${ }^{1} \mathrm{NCN}$ should be observed as the primary, if not exclusive thermal dissociation product.

This qualitative picture has been broadly confirmed by subsequent theoretical studies of $\mathrm{Ci}$ et al. [123] and Pfeifle et al. [124]. The latter authors performed high-level CASPT2 and MRCI calculations and also located the minimum of the crossing seam between $S_{0}$ and $T_{1}$ at $105.4 \mathrm{~kJ} \mathrm{~mol}^{-1}$, which was found to be slightly below the adiabatic dissociation barrier of $107.5 \mathrm{~kJ} \mathrm{~mol}^{-1}$. In order to quantify the possible direct formation of ${ }^{3} \mathrm{NCN}$, a non-adiabatic statistical theory calculation with weak-coupling probabilities has been carried out by Pfeifle et al. [124]. It confirmed that the spin-allowed adiabatic formation of ${ }^{1} \mathrm{NCN}$ dominates, but the spin-forbidden ${ }^{3} \mathrm{NCN}$ formation may contributes to a few percent.

Predominant ${ }^{1} \mathrm{NCN}$ formation in thermal decomposition of $\mathrm{NCN}_{3}$ has been confirmed experimentally as well. Fig. 12 shows singlet and triplet NCN profiles observed by Dammeier et al. [82] in shock-heated diluted mixtures of $\mathrm{NCN}_{3}$ in argon. Here, both ${ }^{1} \mathrm{NCN}$ and ${ }^{3} \mathrm{NCN}$ have been detected by time-resolved narrow-bandwidth absorption in successive experiments. Fast formation of ${ }^{1} \mathrm{NCN}$ was observed, which has been attributed to the unimolecular decomposition of the $\mathrm{NCN}_{3}$ precursor, whereas 
the delayed ${ }^{3} \mathrm{NCN}$ formation shows no indication for a direct ${ }^{3} \mathrm{NCN}$ photodissociation pathway but, as it will be discussed in more detail in Section 5.2, is most consistent with a subsequent singlet-triplet relaxation process.

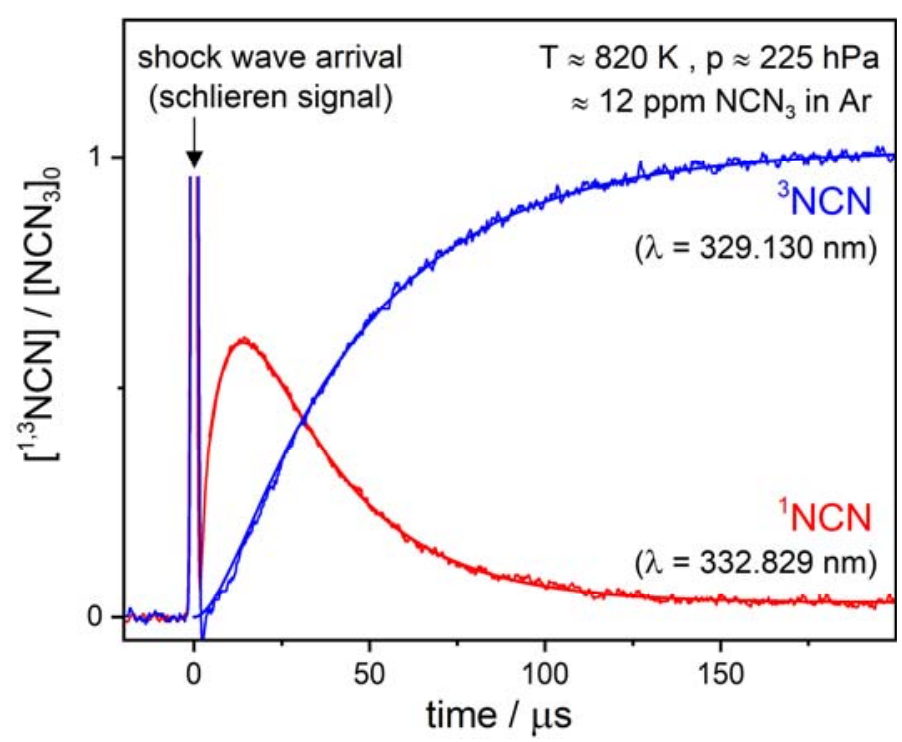

Fig. 12. Singlet and triplet $\mathrm{NCN}$ formation during the thermal decomposition of $\mathrm{NCN}_{3}$ in a shock wave experiment. Data and simulated curves have been adopted from Dammeier et al. [82].

The UV photolysis of $\mathrm{NCN}_{3}$ served as a precursor for many spectroscopic studies $[2,4,5,63,67,88,125,126]$. The absorption peak at $220 \mathrm{~nm}$ corresponds to $S_{1} \leftarrow S_{0}$ excitation [123]. Selective population of the $S_{1}$ state is possible by using the excimer laser wavelength $\lambda=248 \mathrm{~nm}$ [73], whereas at $\lambda=193 \mathrm{~nm}$ both the $S_{1}$ and $S_{2}$ states need to be considered. Calculated electronic transition dipole moments for the $S_{1} \leftarrow S_{0}$ and $S_{2} \leftarrow S_{0}$ transition are $0.24 \mathrm{D}$ and $2.44 \mathrm{D}$, respectively [124].

Depending on the photolysis wavelength, the reported primary photodissociation products are ${ }^{1} \mathrm{NCN}$ as the dominant species under most experimental conditions and $\mathrm{CN}$ in case of short photolysis wavelengths of $\lambda \leq 193 \mathrm{~nm}[125,126]$. Another direct dissociation pathway forming ${ }^{3} \mathrm{NCN}$, as mentioned in Refs. [82,125] for $\lambda \leq 193 \mathrm{~nm}$, points to a potential role of fast ISC processes in photo-excited $\mathrm{NCN}_{3}$. Alternatively, the spin-allowed decomposition of $\mathrm{NCN}_{3}$ yielding excited $\mathrm{N}_{2}$ according to $\mathrm{NCN}_{3} \rightarrow$ ${ }^{3} \mathrm{NCN}\left({ }^{3} \Sigma_{\mathrm{g}}^{-}\right)+\mathrm{N}_{2}\left({ }^{3} \Sigma\right)$ has been suggested $[125,126]$. At room temperature and pressures of a few $\mathrm{hPa}$, however, ${ }^{3} \mathrm{NCN}$ formation is markedly delayed by more than $100 \mu \mathrm{s}$, showing that subsequent processes such as collision-induced ISC and collisional cooling are significant for the overall relaxation process (see also Section 5.2). Theoretical insight into the photodissociation mechanism is provided by the already mentioned high-level computational studies of $\mathrm{Ci}$ et al. [123] and Pfeifle et al. [124]. Consistent with the experimental findings, following photo-excitation from the $S_{0}$ to the $S_{1}$ or $S_{2}$ states of $\mathrm{NCN}_{3}$, 
dominant formation of ${ }^{1} \mathrm{NCN}$ takes place on repulsive potential energy surfaces. As outlined in the work of Pfeifle et al. [124], radiationless transitions of $S_{1}$ and $S_{2}$ to low-lying triplet states or $S_{0}$ may lead to (a minor) direct formation of ${ }^{3} \mathrm{NCN}$ as well, both in its triplet ground state $\tilde{\mathrm{X}}^{3} \Sigma_{\mathrm{g}}^{-}$(via conceivable pathways from $S_{1}$ and $S_{2}$ ) and in its excited $\tilde{\mathrm{A}}^{3} \Pi_{\mathrm{u}}$ state (via pathways from $S_{2}$ ).

\section{The role of the spin state of NCN}

As already mentioned in Section 2 (see Fig. 2), the electronic ground state of NCN is a ${ }^{3} \Sigma_{\text {g }}^{-}$state. The next higher and lowest singlet state is a ${ }^{1} \Delta_{\mathrm{g}}$ state located $(8146 \pm 80) \mathrm{cm}^{-1}$ above the ground state, hence corresponding to a singlet-triplet splitting of $\Delta_{\mathrm{ST}} H^{\circ}{ }_{0}=(97.5 \pm 1.0) \mathrm{kJ} \mathrm{mol}^{-1}$ [64]. As the reactivity of singlet and triplet states can be very different, the question for possible mechanistic consequences in combustion systems arises as well as the question for the mechanism and kinetics of the ISC process. This section reviews the current state of knowledge regarding these topics.

\subsection{The potential role of ${ }^{1} \mathrm{NCN}$ in thermal systems}

In thermal systems, the relative population of the singlet state is lower than $1 \%$ up to temperatures of $2650 \mathrm{~K}$. Thus, ${ }^{1} \mathrm{NCN}$ chemistry would not be expected to play an important role in prompt-NO formation and has not been considered in flame models so far. However, for those NCN reactions that are comparatively slow, for example reactions with closed-shell species, ${ }^{1} \mathrm{NCN}$ chemistry might become significant as is shown by the following example: Assuming a fast thermal equilibration ${ }^{3} \mathrm{NCN} \rightleftharpoons{ }^{1} \mathrm{NCN}$ with an equilibrium constant $K_{\mathrm{eq}}$ calculated from the NASA polynomials as reported in this work (see Tables S1 and S4 of the Supplementary Material), the ${ }^{1} \mathrm{NCN}$-related fraction $\phi_{\text {singlet }}$ of the overall NCN loss caused by a pair of ${ }^{1} \mathrm{NCN}+\mathrm{X}$ and ${ }^{3} \mathrm{NCN}+\mathrm{X}$ reactions,

$$
\phi_{\text {singlet }}=\frac{\mathrm{d}\left(\left[{ }^{1} \mathrm{NCN}\right]\right)}{\mathrm{d}([\mathrm{NCN}])}=\frac{k_{\text {singlet }}\left[{ }^{1} \mathrm{NCN}\right]}{k_{\text {singlet }}\left[{ }^{1} \mathrm{NCN}\right]+k_{\text {triplet }}\left[{ }^{3} \mathrm{NCN}\right]}
$$

can be rearranged to an expression for the temperature-dependent ratio of the singlet and triplet reaction rate constants as a function of $\phi_{\text {singlet: }}$

$$
\frac{k_{\text {singlet }}}{k_{\text {triplet }}}=\frac{\phi_{\text {singlet }}}{\left(1-\phi_{\text {singlet }}\right) K_{\text {eq }}}
$$


Fig. 13 plots the rate constant ratio corresponding to a fraction $\phi_{\text {singlet }}=1 \%($ or $10 \%, 50 \%, 90 \%)$ as a function of temperature. For example, supposing that $k_{\text {singlet }} / k_{\text {triplet }}=1000$, already at a temperature of $1135 \mathrm{~K}$ (or $1485 \mathrm{~K}, 2070 \mathrm{~K}, 3420 \mathrm{~K}), 1 \%(10 \%, 50 \%, 90 \%)$ of the overall NCN loss would be due to the ${ }^{1} \mathrm{NCN}$ reaction. Clearly, separate treatment of ${ }^{1} \mathrm{NCN}$ and ${ }^{3} \mathrm{NCN}$ chemistry may be necessary at such temperatures, in particular in case of different products for the singlet and triplet reaction. Note, however, that many bimolecular reactions of ${ }^{3} \mathrm{NCN}$ with atoms and radical species exhibit rate constant values on the order of $10^{13}-10^{14} \mathrm{~cm}^{3} \mathrm{~mol}^{-1} \mathrm{~s}^{-1}$. In those cases, separate inclusion of ${ }^{1} \mathrm{NCN}$ chemistry will not change the overall picture very much because the ${ }^{1} \mathrm{NCN}$ reaction can be only about a factor of 10 faster at maximum (otherwise the rate constant would exceed the collision number).

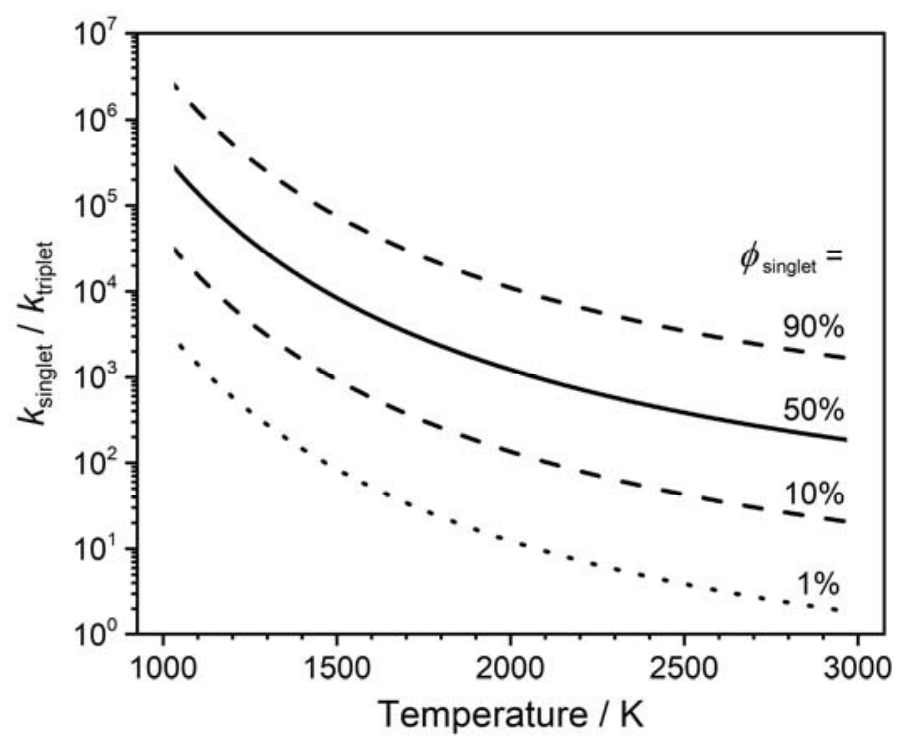

Fig. 13. Role of ${ }^{1} \mathrm{NCN}$ chemistry in thermal systems. The plot shows the ratio of the rate constants of two corresponding singlet and triplet $\mathrm{NCN}$ reactions, ${ }^{1,3} \mathrm{NCN}+\mathrm{X}$, that result in a singlet contribution of $\phi_{\text {singlet }}=1 \%$, $10 \%, 50 \%$, and $90 \%$ to the overall NCN loss.

In general, excited singlet radical chemistry is fundamentally different from ground state triplet chemistry. For example, ground state ${ }^{3} \mathrm{CH}_{2}\left(\tilde{\mathrm{X}}^{3} \mathrm{~B}_{1}\right)$ reacts with hydrocarbons non-stereospecific and activation-controlled under $\mathrm{H}$ atom abstraction, whereas excited ${ }^{1} \mathrm{CH}_{2}\left(\tilde{\mathrm{a}}^{1} \mathrm{~A}_{1}\right)$ inserts in single bonds and undergoes stereospecific addition to double bonds with no or very low energy barriers and rate coefficients close to collision number [127-129]. Similarly, ${ }^{1} \mathrm{NH}\left(\mathrm{a}^{1} \Delta\right)$ inserts into $\mathrm{CH}$ bonds, whereas ${ }^{3} \mathrm{NH}\left(\mathrm{X}^{3} \Sigma^{-}\right)$abstracts hydrogen from hydrocarbons, $\mathrm{H}_{2}$, and $\mathrm{H}_{2} \mathrm{O}$ [130,131]. So far, examples for the explicit consideration of ${ }^{1} \mathrm{NCN}$ chemistry in reaction kinetics studies, next to the singlet-triplet quenching discussed below, are scarce.

In their shock tube study on the reaction $\mathrm{NCN}+\mathrm{O}_{2}$, Faßheber and Friedrichs [114] postulated the fast formation of a ${ }^{3} \mathrm{NCN}-\mathrm{OO}$ species from the reaction ${ }^{1} \mathrm{NCN}+\mathrm{O}_{2}\left({ }^{3} \Sigma_{\mathrm{g}}^{-}\right)$. The formation of ${ }^{3} \mathrm{NCN}-\mathrm{OO}$ 
served as a temporary sink for NCN that was needed to explain the experimentally observed delayed formation of ${ }^{3} \mathrm{NCN}$ in shock-heated mixtures of $\mathrm{NCN}_{3} / \mathrm{O}_{2} / \mathrm{Ar}$. The delayed ${ }^{3} \mathrm{NCN}$ formation has been modeled by a subsequent, independent decomposition step ${ }^{1} \mathrm{NCNOO}^{*} \rightarrow{ }^{3} \mathrm{NCN}+\mathrm{O}_{2}\left({ }^{3} \Sigma_{\mathrm{g}}^{-}\right)$, hence implicitly assuming a rate-limiting ISC step forming ${ }^{1} \mathrm{NCNOO}^{*}$ in-between. According to their data, the ${ }^{1} \mathrm{NCN}+\mathrm{O}_{2} \rightarrow{ }^{3} \mathrm{NCN}-\mathrm{OO}$ reaction proceeds with a rate constant $>600$ times higher than that of the ${ }^{3} \mathrm{NCN}$ $+\mathrm{O}_{2}$ reaction (calculated for $T=1500 \mathrm{~K}$ and $p=25 \mathrm{kPa}$ ). Consequently, the singlet reaction may be competitive under combustion conditions albeit the fate of the postulated ${ }^{3} \mathrm{NCN}-\mathrm{OO}$ species remains unclear.

More recently, Faßheber et al. [80] performed a quantum chemical kinetic study on the products of the reaction ${ }^{1,3} \mathrm{NCN}+\mathrm{H}_{2}$ (see Section 6.2.3). Similar to the trends observed for ${ }^{1,3} \mathrm{CH}_{2}$ and ${ }^{1,3} \mathrm{NH}$, the ${ }^{3} \mathrm{NCN}$ reaction yields the $\mathrm{H}$ abstraction product $\mathrm{HNCN}+\mathrm{H}$ whereas the ${ }^{1} \mathrm{NCN}$ was found to form the $\mathrm{N}$-centered insertion product $\mathrm{NCNH}_{2}$ (followed by isomerization to $\mathrm{HNCNH}$ and decomposition to $\mathrm{HNCN}+\mathrm{H})$. In contrast to the ${ }^{1} \mathrm{NH}$ and ${ }^{1} \mathrm{CH}_{2}$ reactions that proceed without energy barrier and close to the collision limit, a sizeable entrance barrier of $74 \mathrm{~kJ} \mathrm{~mol}^{-1}$ has been found for the ${ }^{1} \mathrm{NCN}$ reaction (in comparison with a barrier of $115 \mathrm{~kJ} \mathrm{~mol}^{-1}$ for the corresponding ${ }^{3} \mathrm{NCN}$ reaction). This reaction barrier has been attributed to the necessary reorganization of the HOMO electron density during the bond insertion that is initially uniformly distributed over the two $\mathrm{N}$ atom sites in ${ }^{1} \mathrm{NCN}$ [80]. Nevertheless, the rate constant of the singlet reaction was found to be 600 times larger than for ${ }^{3} \mathrm{NCN}$ at $T=1000 \mathrm{~K}$ and taking into account the singlet reaction significantly improved the agreement between experimental shock tube data and theoretical predictions. For implementing the reaction $\mathrm{NCN}+\mathrm{H}_{2}$ into flame models, they recommended either to consider ${ }^{1} \mathrm{NCN}$ and ${ }^{3} \mathrm{NCN}$ chemistry separately or to use an effective rate constant expression for $\mathrm{NCN}$, treating $\mathrm{NCN}$ as a sum species by properly weighting over the singlet and triplet contributions. This is sufficiently accurate as the main reaction products in case of the $\mathrm{H}_{2}$ reaction are the same and the population of the ${ }^{1} \mathrm{NCN}$ contribution under thermal equilibrium conditions remains small anyway.

\subsection{Singlet-triplet relaxation}

${ }^{1} \mathrm{NCN}$ is the most likely initial species formed in kinetic experiments. Therefore, the timescales and mechanisms of singlet-triplet relaxation are important to properly evaluate the experiments. The singlet and triplet NCN profiles observed by Dammeier et al. [82] after shock-heating diluted mixtures of $\mathrm{NCN}_{3}$ in argon (Fig. 12) reveal that the fast formation of ${ }^{1} \mathrm{NCN}$ (red curve) from the unimolecular decomposition of $\mathrm{NCN}_{3}$ is followed by a subsequent ${ }^{1} \mathrm{NCN}$ decay taking place on about the same timescale as the observed delayed ${ }^{3} \mathrm{NCN}$ formation (blue curve). The delayed formation of the triplet species was interpreted by Dammeier et al. [82] in terms of a rate-limiting collision-induced intersystem crossing (CIISC) step, ${ }^{1} \mathrm{NCN}+(\mathrm{M}) \rightarrow{ }^{3} \mathrm{NCN}+(\mathrm{M})$. CIISC was found to be pressure dependent with a 
temperature dependence resulting in an activation energy of about $24-36 \mathrm{~kJ} \mathrm{~mol}^{-1}$. Addition of a collider gas different from argon, namely $\mathrm{NO}, \mathrm{NO}_{2}$ and $\mathrm{O}_{2}$, significantly accelerated the CIISC dynamics; factors of 40 and 400 higher CIISC efficiencies have been reported for $\mathrm{NO}$ and $\mathrm{NO}_{2}$, respectively [112,114]. Dammeier et al. [82] also measured ${ }^{1} \mathrm{NCN}$ and ${ }^{3} \mathrm{NCN}$ profiles in room temperature $193 \mathrm{~nm}$ photolysis experiments of $\mathrm{NCN}_{3}$. Again, fast ${ }^{1} \mathrm{NCN}$ and delayed ${ }^{3} \mathrm{NCN}$ formation has been observed. Here, the fast initial increase of the observed ${ }^{1} \mathrm{NCN}$ profiles has been attributed to the collisional relaxation of electronically and vibrationally excited ${ }^{1} \mathrm{NCN}^{*}$ (* denotes the vibrational excitation). The somewhat delayed, more complex ${ }^{3} \mathrm{NCN}$ formation profiles with a fast and a slow apparent time constant (the latter consistent with the measured ${ }^{1} \mathrm{NCN}$ decay) have been assigned to ${ }^{3} \mathrm{NCN}^{*}$ relaxation (of a rapidly formed triplet photodissociation product) and CIISC of thermalized ${ }^{1} \mathrm{NCN}$.

In order to further clarify the mechanism responsible for the delayed ${ }^{3} \mathrm{NCN}$ formation, Hetzler and Olzmann [73] performed an extensive study of the $248 \mathrm{~nm}$ photolysis of $\mathrm{NCN}_{3}$ over a temperature range of 240-293 $\mathrm{K}$ and a pressure range of 1-80 $\mathrm{kPa}$ with different bath gases including $\mathrm{Ar}, \mathrm{Ne}, \mathrm{Kr}, \mathrm{H}_{2}, \mathrm{O}_{2}$, and $\mathrm{N}_{2} \mathrm{O}$. The reported temperature and pressure dependences were found to be consistent with a Landau-Teller/Schwartz-Slawsky-Herzberg model for V-T energy transfer, and a two-channel vibrational relaxation model was suggested. In contrast to the interpretation of Dammeier et al. [82], in the model of Ref. [73] the slow component of ${ }^{3} \mathrm{NCN}$ was not attributed to CIISC but to a slow vibrational relaxation in the triplet manifold. This implies the assumption of a prior fast ISC process. An interpretation with such an efficient singlet-triplet ISC pathway seems to be inconsistent with the reported high ${ }^{1} \mathrm{NCN}$ concentration levels in the work of Dammeier et al. [82]. We note, however, that a one-to-one comparison of the underlying $248 \mathrm{~nm}$ and $193 \mathrm{~nm}$ experiments is not warranted because of the much higher excitation energy in the $193 \mathrm{~nm}$ experiments that may populate higher electronic states with different dynamics. A possible dependence of the $\mathrm{NCN}$ spin multiplicity on the $\mathrm{NCN}_{3}$ photolysis wavelength was already discussed in very early works $[6,132]$. Clearly, the interpretation of the initial dynamics of the excited ${ }^{1} \mathrm{NCN}$ and ${ }^{3} \mathrm{NCN}$ species remains an open question. In order to shed light into these puzzling relaxation processes, Pfeifle et al. [124] performed a very detailed and so far most comprehensive theoretical investigation of the ISC mechanism in this reaction system. Based on multireference configuration interaction calculations, they conclude with the surprising result that the experimental, relatively fast time scales ( $\mu$ s to ms range) for $\tilde{a}^{1} \mathrm{NCN} \rightarrow \tilde{\mathrm{X}}^{3} \mathrm{NCN}$ intersystem crossing can be explained neither by ISC channels resulting from surface crossings nor by mixed-multiplicity rovibrational gateway states. According to their work, surface crossing occurs at energies too high to be accessible under the typical experimental conditions, and spin-orbit interactions are generally weak due to the large singlet-triplet splitting. For example, the surface crossing with the lowest energy barrier between the $\tilde{a}^{1} \Delta_{\mathrm{g}}$ and the $\tilde{\mathrm{X}}^{3} \Sigma_{\mathrm{g}}^{-}$state has been identified in the bending coordinate at an energy of $156 \mathrm{~kJ}$ $\mathrm{mol}^{-1}$ (relative to the singlet minimum), that is by far too high to explain the fast CIISC observed under 
thermal conditions. As outlined by Pfeifle et al. [124], future time- and state-resolved studies are needed to untangle the different superimposed processes.

\section{Rate constants of NCN reactions from theory and experiment}

Despite the possible interference of ${ }^{1} \mathrm{NCN}$ chemistry, theoretical and experimental studies have focused on the theoretical prediction and direct measurement of the rate constants of ${ }^{3} \mathrm{NCN}$ elementary reactions. From the experimental point of view, in particular for high-temperature studies and at elevated pressures, relaxation is fast and temperature is usually still low enough to ensure dominating triplet chemistry. Nevertheless, one should keep in mind that in most cases the experimentally measured rate constant represents an appropriate thermal average and implicitly includes the minor or negligible contribution of the corresponding ${ }^{1} \mathrm{NCN}$ reaction. Known exceptions have been already discussed in Section 5.1.

Consequently, in the following sections on existing reaction kinetics work, unless otherwise stated, we continue to use "NCN" when referring to triplet $\mathrm{NCN}$ or at least a triplet NCN dominated overall reaction.

\subsection{Reactions involved in the $\mathrm{CHN}_{2}$ network}

As already outlined in the Introduction, illustrated in the schematic potential energy diagram in Fig. 1 and confirmed by time-resolved measurements of $\mathrm{CH}$ at low pressure [21,133] as well as theoretical calculations based on TST-RRKM models [21,24], at low temperature $(T<800 \mathrm{~K})$ the reaction between $\mathrm{CH}$ and $\mathrm{N}_{2}$ yields an $\mathrm{HCNN}$ adduct through a pressure-dependent association step along the doublet surface (left direction in Fig. 1). At intermediate temperature (800-1800 K), this HCNN adduct rapidly dissociates back to $\mathrm{CH}+\mathrm{N}_{2}[22,24]$. At the same time, the activation-controlled reactions forming other $\mathrm{CHN}_{2}$ intermediates and $\mathrm{NCN}+\mathrm{H}$ on the doublet surface (right direction in Fig. 1) start to prevail. Becker et al. [133] could observe the related change of the temperature dependence of the overall rate constant (from negative to positive) for $T>673 \mathrm{~K}$.

\subsubsection{The reaction $\mathrm{CH}+\mathrm{N}_{2} \rightleftharpoons \mathrm{NCN}+\mathrm{H}$}

At high temperature, $\mathrm{NCN}$ formation through the reaction between $\mathrm{CH}$ and $\mathrm{N}_{2}$ was first shown to take place on the doublet surface in quantum chemical calculations (mainly at G2M(RCC) level of theory) by Moskaleva et al. [7,8]. In the same breath, Mosakaleva et al. [7,8] postulated that the previously assumed spin-forbidden direct formation of $\mathrm{HCN}+\mathrm{N}(\mathrm{R} 1 \mathrm{~b})$ should be obsolete. Instead, the 
spin-allowed reaction products $\mathrm{NCN}+\mathrm{H}(\mathrm{R} 1 \mathrm{a})$, in subsequent bimolecular steps, can yield $\mathrm{HCN}+\mathrm{N}$ along a quartet pathway or, alternatively, yield back $\mathrm{CH}+\mathrm{N}_{2}$ through the reverse reaction on the doublet surface (see Fig. 1). However, the initial rate constant estimates for reaction (R1a) calculated with a multichannel variational RRKM approach in Ref. [7] turned out to be too low to adequately reproduce the measured NO species profiles in low-pressure flames as shown in Ref. [33]. As a consequence, merely the product channel (R1a) of the reaction $\mathrm{CH}+\mathrm{N}_{2}$ has been implemented into the GDFkin ${ }^{\circledR} 3.0 \_$NCN model [33] with the high-temperature rate constant adopted from the shock tube measurements of Lindackers et al. [134].

Vasudevan et al. [31] determined the rate constant of the $\mathrm{CH}+\mathrm{N}_{2}$ reaction in a shock tube by using $\mathrm{CH}$ and $\mathrm{NCN}$ laser absorption. In a perturbation type of approach, $\mathrm{CH}$ concentration-time profiles have been measured with and without the addition of nitrogen to the reaction mixtures, where the observed perturbation of the $\mathrm{CH}$ profile upon nitrogen addition was principally assigned to the $\mathrm{CH}+\mathrm{N}_{2}$ reaction. The experiments were primarily designed to prove that $\mathrm{NCN}$ is the predominant product of the reaction $\mathrm{CH}+\mathrm{N}_{2}$. For the temperature range $1950-3550 \mathrm{~K}$, the authors reported a rate constant expression as given in Table 4. Based on a detailed analysis of the $\mathrm{CH}$ concentration-time profiles, they were also able to show that the branching fraction $k_{1 \mathrm{a}} /\left(k_{1 \mathrm{a}}+k_{1 \mathrm{~b}}\right)$ should be close to 1 , with a lower limit of 0.7 .

Soon after, Harding et al. [15] performed theoretical calculations with multi-reference methods, showing that the single-reference approach previously used by Moskaleva and Lin [7] gives rise to large uncertainties. The rate constant for reaction (R1a) calculated in Ref. [15] (see Table 4 and Fig. 14) werewas found in very good agreement with the experimental values reported by Vasudevan et al. [31]. The dominant sources of uncertainty in their calculations were attributed to the energy of the saddle point connecting the cyclic $\mathrm{HCN}_{2}$ structure with the open-chain $\mathrm{HNCN}$ structure (see Fig. 1), which was reported with an accuracy of $\pm 8.4 \mathrm{~kJ} \mathrm{~mol}^{-1}$, and to the enthalpy of formation of NCN, which was calculated to be about $7-8 \mathrm{~kJ} \mathrm{~mol}^{-1}$ higher than the current best estimate of $\Delta_{\mathrm{f}} H^{\circ}(\mathrm{NCN})=451 \mathrm{~kJ} \mathrm{~mol}^{-}$ ${ }^{1}$ (see Section 3.3). Harding et al. [15] estimated the related uncertainty in $k_{1 \mathrm{a}}$ to a factor of 2.4, 1.4 and 1.2 at 1000,2000 and $3000 \mathrm{~K}$, respectively.

In 2013, Teng et al. [16] recalculated rate constants of NCN $+\mathrm{H}$ reactions based on ab initio molecular-orbital theory at $\operatorname{CCSD}(\mathrm{T}) / \mathrm{CBS} / 6-311++\mathrm{G}(3 \mathrm{df}, 2 \mathrm{p})$ level and an RRKM model. They considered the reaction pathways from $\mathrm{NCN}+\mathrm{H}$ yielding $\mathrm{CH}+\mathrm{N}_{2}$ (R-1a) and HNCN (R3) along the doublet surface as well as $\mathrm{HCN}+\mathrm{N}(\mathrm{R} 2)$ and $\mathrm{HNC}+\mathrm{N}(\mathrm{R} 4)$ along the quartet surface (see Fig. 1). The authors give rate constants for these four reactions and also mention that the overall rate constant for $\mathrm{CH}+\mathrm{N}_{2}$ previously proposed by two of the authors [7] was underestimated due to an error in their RRKM code. The updated value of the rate constant $k_{\text {la }}$ (based on $\left.\Delta_{\mathrm{f}} H^{\circ}{ }_{0}(\mathrm{NCN})=458 \mathrm{~kJ} \mathrm{~mol}^{-1}\right)$ is in good agreement with the results of Harding et al. [15]. 
Lamoureux et al. $[44,52,135,136]$ modeled, with a revised prompt-NO mechanism, species profiles of $\mathrm{CH}, \mathrm{NO}, \mathrm{HCN}, \mathrm{NCN}$, and $\mathrm{NCO}$ experimentally determined in low-pressure flames. Starting from the values proposed by Vasudevan et al. [31] and Harding et al. [15], the rate constant $k_{1 \text { a }}$ was adjusted to reasonably reproduce the species profiles.

Very recently, Klippenstein et al. [17] reanalyzed the $\mathrm{CHN}_{2}$ reaction network with further advanced quantum chemical and statistical rate theory calculations. Among others, also the aforementioned reaction channels (R-1a), (R2), (R3), and (R4) were considered. Based on thermodynamic parameters from the more accurate ANL1 method (Table 3) and a detailed master equation modeling, the complex pressure-dependence of the different reaction channels was examined. All pressure-dependent rate constants were reported in Ref. [17] in PLOG format.

\section{Table 4}

Temperature dependence of the rate constant for the reaction $\mathrm{CH}+\mathrm{N}_{2} \rightarrow \mathrm{NCN}+\mathrm{H}$ (R1a), expressed in the form $k_{1 \mathrm{a}}=A(T / \mathrm{K})^{n} \exp (-E / R T)$, and reported $\Delta_{\mathrm{f}} H^{\circ}{ }_{298}(\mathrm{NCN})$ values.

\begin{tabular}{|c|c|c|c|c|c|c|}
\hline $\begin{array}{l}A / \\
\mathrm{cm}^{3} \mathrm{~mol}^{-1} \mathrm{~s}^{-1}\end{array}$ & $n$ & $\begin{array}{l}E / \\
\mathrm{kJ} \mathrm{mol}^{-1}\end{array}$ & $\begin{array}{l}T / \\
\mathrm{K} \\
\end{array}$ & $\begin{array}{l}p / \\
\mathrm{kPa}\end{array}$ & $\begin{array}{l}\Delta_{\mathrm{f}} H^{\circ}{ }_{298}(\mathrm{NCN}) / \\
\mathrm{kJ} \mathrm{mol}^{-1}\end{array}$ & Refs. \\
\hline $2.22 \times 10^{7}$ & 1.48 & 97.767 & $1500-4000$ & $50-200$ & 464.0 & Moskaleva and Lin [7] \\
\hline $5.1 \times 10^{11}$ & 0 & 56.9 & & & 450.2 & El Bakali et al. [33 ${ }^{\mathrm{a}}$ \\
\hline $6.03 \times 10^{12}$ & 0 & 92.5 & $1943-3543$ & $90-140$ & 465.0 & Vaseduvan et al. [31] \\
\hline $4.09 \times 10^{8}$ & 1.122 & 73.32 & $400-3000$ & & 458.2 & Harding et al. [15] \\
\hline $1.35 \times 10^{9}$ & 0.90 & 72.89 & $800-4000$ & 100 & 457.9 & Teng et al. [16] \\
\hline $1.95 \times 10^{12}$ & 0.0 & 70.772 & & & 444.3 & Lamoureux et al. $[52]^{\mathrm{a}}$ \\
\hline $2.5 \times 10^{9}$ & 0.89 & 69.54 & $500-2500$ & 100 & 450.8 & Klippenstein et al. [17] ${ }^{\mathrm{b}}$ \\
\hline
\end{tabular}

${ }^{a}$ fitted from shock tube experiments and validated for flame modeling at low- and high-pressure

$\mathrm{b}$ the pressure-dependent rate constant is given at $p=0.1 \mathrm{MPa}$.

Rate constant expressions for the reaction $\mathrm{CH}+\mathrm{N}_{2} \rightarrow \mathrm{NCN}+\mathrm{H}$ (R1a) from the literature are collected in Table 4 along with the values of the NCN enthalpy of formation that have been adopted in the corresponding references. The rate constants $k_{1 \mathrm{a}}$ are plotted in Arrhenius form in Fig. 14. For clarity, the values reported in Refs. [7,33] are omitted because they considerably differ from the others. The experimental values reported by Vasudevan et al. [31] (symbols and black dotted line) are compared to results of theoretical calculations from Refs. [15-17] (green, black and blue solid lines) and to values indirectly determined from flame modeling [52] (red dot-dashed line). The values for $k_{\text {la }}$ vary by more than a factor of four at $1000 \mathrm{~K}$, but are closer to each other and in agreement with the shock tube data from Vasudevan et al. [31] at higher temperatures (around 2500 K) (see Fig. S3 of the Supplementary Material for a magnification of Fig. 14). Originally, Harding et al. [15] determined a value of $\Delta_{\mathrm{f}} H^{\circ} 0(\mathrm{NCN})=459 \mathrm{~kJ} \mathrm{~mol}^{-1}$. According to their analysis of possible uncertainties, the now recommended 
lower value $\Delta_{\mathrm{f}} H^{\circ}{ }_{0}(\mathrm{NCN})=451 \mathrm{~kJ} \mathrm{~mol}^{-1}$ (see Section 3.3) would increase their rate constant $k_{1 \mathrm{a}}$ by factors of $1.8,1.25$, and 1.1 at $T=1000 \mathrm{~K}, 1500 \mathrm{~K}$, and $2000 \mathrm{~K}$, respectively, while it does almost not affect the rate constant at even higher temperature. The very recent pressure-dependent rate constant of Klippenstein et al. [17] at $p=0.1 \mathrm{MPa}$ (based on $\Delta_{\mathrm{f}} H^{\circ}{ }_{0}(\mathrm{NCN})=450.2 \mathrm{~kJ} \mathrm{~mol}^{-1}$ ) is very close to the result of their previous calculations [15] when referenced to the same enthalpy of formation. Note, however, that according to their calculations the product branching ratios changed because the collisional stabilization of the HNCN intermediate was found to become important at low temperatures and high pressures. For example, whereas at $p=0.1 \mathrm{MPa}$ and $T=1500 \mathrm{~K}$, the relative branching fraction predicted for the $\mathrm{HNCN}$ channel is only $4 \%$, it increases to $62 \%$ at $10 \mathrm{MPa}$ at the same temperature. Overall, the good agreement of the results from all recent theoretical studies reveals a reliable prediction of the rate constant $k_{1 \mathrm{a}}$ and, together with the recommended value of the NCN enthalpy of formation, of the rate constant $k_{-1 \text { a }}$ of the reverse reaction as well.

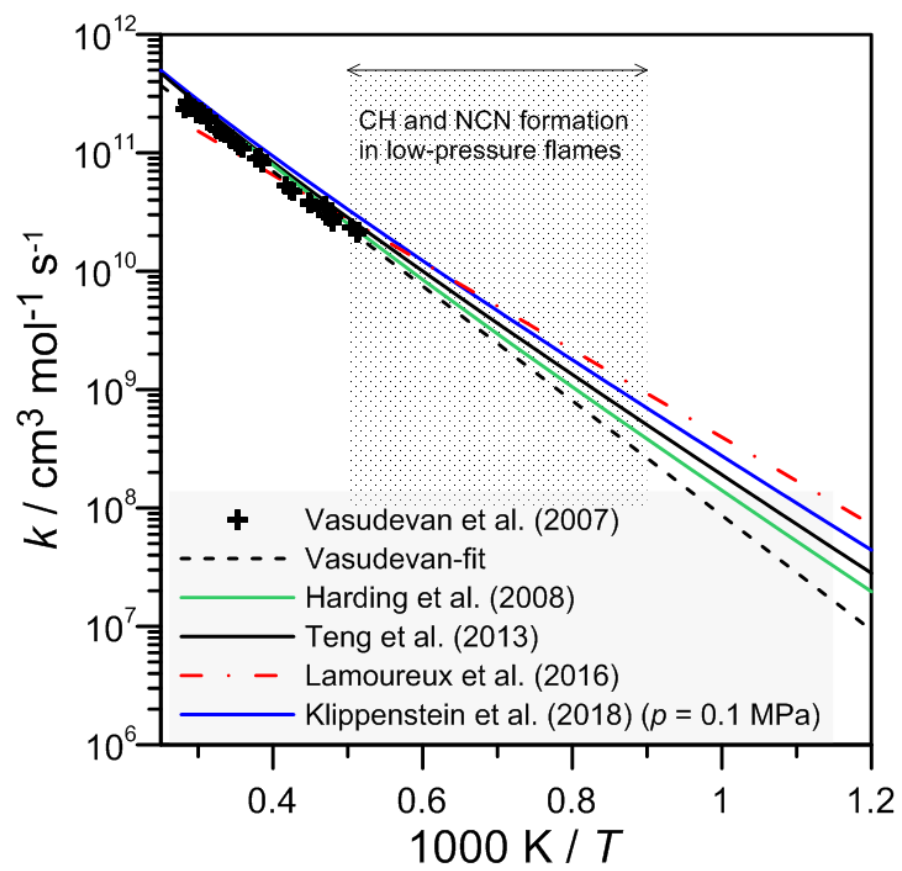

Fig. 14. Arrhenius plot of the rate constant for reaction $\mathrm{CH}+\mathrm{N}_{2} \rightarrow \mathrm{NCN}+\mathrm{H}$ (R1a) according to Vasudevan et al. [31], Harding et al. [15], Teng et al. [16], Lamoureux et al. [52], and Klippenstein et al. [17] at $p=0.1 \mathrm{MPa}$. The dotted pattern indicates the temperature range where the $\mathrm{CH}$ and $\mathrm{NCN}$ species co-exist in flame environment. See also a magnification in the SM (Fig. S3).

The importance of the NCN enthalpy of formation (governing the temperature dependence of the related equilibrium constants) to calculate the rate constant of the reverse reaction (R-1a) in detailed kinetic models for NO prediction is further illustrated in Fig. 15. Here, the Arrhenius plot shows the rate constants $k_{\text {-1a }}(T)$ used or predicted by different authors; the corresponding expressions are listed in Table 5. The curves present the values of $k_{\text {-1a }}$ obtained from theoretical calculations $[16,17]$ (solid and dotted 
lines), from kinetic modeling of shock tube data [102] (dashed line), and from flame modeling [52] (dotdashed line). The rate constant determined by Faßheber et al. [102] is a factor of about 0.7 lower than those derived by Klippenstein et al. [17], but with a very similar temperature dependence. In fact, the rate constant from Faßheber et al. [102] was derived from the calculated rate constant $k_{1 \text { a }}$ proposed by Harding et al. [15] by adjusting the enthalpy of formation of NCN to a value of $\Delta_{\mathrm{f}} H^{\circ}{ }_{298}(\mathrm{NCN})=450 \mathrm{~kJ}$ $\mathrm{mol}^{-1}$ and by applying a scaling factor of 0.65 to fit their own experimental shock tube data for the overall reaction $\mathrm{NCN}+\mathrm{H}$ (see Section 6.1.2). At $T=1650 \mathrm{~K}$, the rate constant $k_{\text {-1a }}$ calculated from the value of $k_{1 \text { a }}$ proposed by Lamoureux et al. [52], that was based on $\Delta_{\mathrm{f}} H^{\circ}{ }_{298}(\mathrm{NCN})=444.3 \mathrm{~kJ} \mathrm{~mol}^{-1}$, is a factor of about 0.5 lower than $k_{\text {-1a }}$ from Ref. [17]. On the other hand, the value reported by Teng et al. [16], with $\Delta_{\mathrm{f}} H^{\circ}{ }_{0}(\mathrm{NCN})=457.9 \mathrm{~kJ} \mathrm{~mol}^{-1}$, is a factor of 3.5 above the value determined by Klippenstein et al. [17]. From these considerations, it becomes obvious that a consistent dataset of $k_{1 \mathrm{a}}(T, p), k_{\text {-1a }}(T, p)$ and $\Delta_{\mathrm{f}} H^{\circ}(\mathrm{NCN})(T)$ is of utmost importance for a proper modeling of prompt-NO formation. This will be further outlined in Section 8 .

\section{Table 5}

Temperature dependence of the rate constant for the reaction $\mathrm{NCN}+\mathrm{H} \rightarrow \mathrm{CH}+\mathrm{N}_{2}$ (R-1a), expressed in the form $k_{\text {-1a }}=A(T / \mathrm{K})^{n} \exp (-E / R T)$.

\begin{tabular}{|l|l|l|l|l|l|}
\hline$A / \mathrm{cm}^{3} \mathrm{~mol}^{-1} \mathrm{~s}^{-1}$ & $n$ & $E / \mathrm{kJ} \mathrm{mol}^{-1}$ & $T / \mathrm{K}$ & $p / \mathrm{kPa}$ & Refs. \\
\hline $3.77 \times 10^{19}$ & -1.61 & 6.23 & $298-3000$ & $6-9870$ & Teng et al. [16] $^{\mathrm{a}}$ \\
\hline $4.2 \times 10^{15}$ & -0.69 & 2.0 & $962-2425$ & $28-213$ & Faßheber et al. [102] $^{\mathrm{b}}$ \\
\hline $6.204 \times 10^{16}$ & -1.007 & 2.100 & & & Lamoureux et al. [52] $^{\mathrm{c}}$ \\
\hline $7.6 \times 10^{15}$ & -0.68 & 1.243 & $500-2500$ & 100 & Klippenstein et al. [17] $^{\mathrm{d}}$ \\
\hline
\end{tabular}

${ }^{\mathrm{a}}$ theoretical study, no pressure dependence

${ }^{\mathrm{b}}$ recommended rate constant expression based on $\Delta_{\mathrm{f}} H^{\circ}{ }_{298}(\mathrm{NCN})=450 \mathrm{~kJ} \mathrm{~mol}^{-1}$

${ }^{\mathrm{c}}$ calculated from the direct expression $k_{1 \mathrm{a}}$ [52] and the equilibrium constant using ChemRev [137]

${ }^{\mathrm{d}}$ data in PLOG format for $1 \mathrm{kPa}<p<10 \mathrm{MPa}$ are given in the Suppl. Mat. of Ref. [17] 


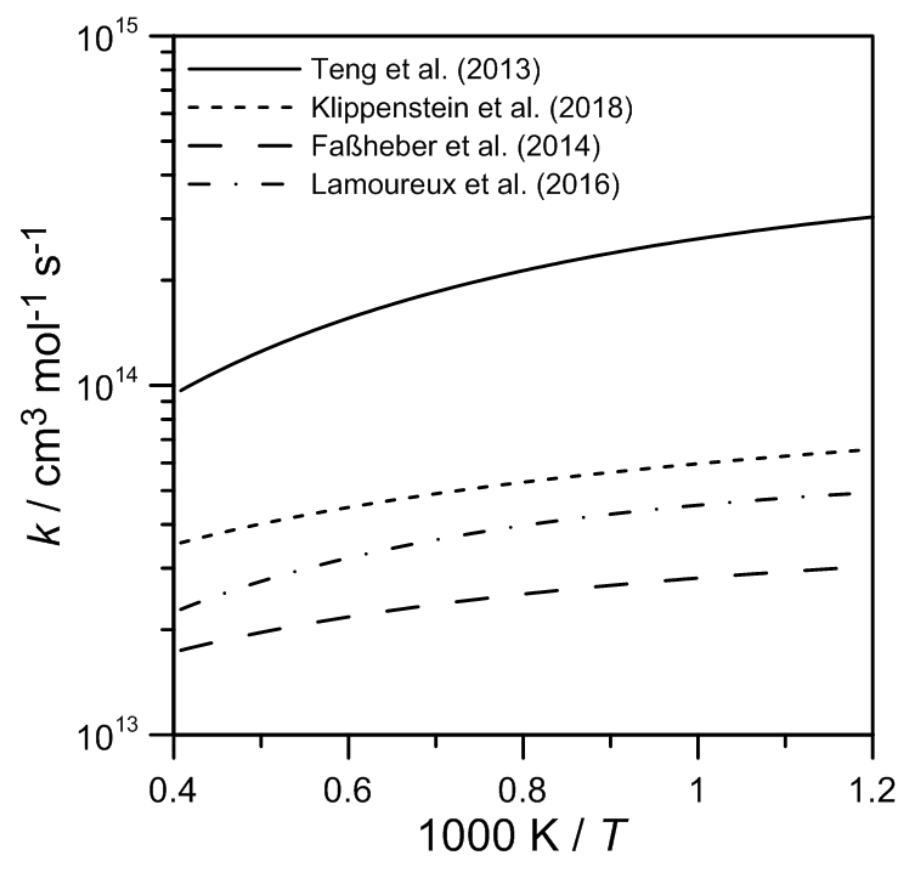

Fig. 15. Arrhenius plot of the rate constants for the reaction $\mathrm{NCN}+\mathrm{H} \rightarrow \mathrm{CH}+\mathrm{N}_{2}$ (R-1a) calculated by Teng et al. [16] and (at $p=100 \mathrm{kPa}$ ) Klippenstein et al. [17]. Also indicated are the rate constants determined by Faßheber et al. [102] and Lamoureux et al. [52].

\subsubsection{The reactions $N C N+H \rightarrow$ products}

According to the theoretical works by Teng et al. [16] and Klippenstein et al. [17], the reaction NCN $+\mathrm{H}$ can proceed on a doublet or a quartet potential energy surface (see Fig. 1). On the doublet surface, either the recombination product $\mathrm{HNCN}$ (R3) can be formed or, via a number of subsequent slightly submerged barriers lying $15-37 \mathrm{~kJ} \mathrm{~mol}^{-1}$ below the $\mathrm{NCN}+\mathrm{H}$ entrance energy, the products $\mathrm{CH}+\mathrm{N}_{2}$ (R-1a). On the quartet surface, $\mathrm{HCN}+\mathrm{N}(\mathrm{R} 2)$ or $\mathrm{HNC}+\mathrm{N}(\mathrm{R} 4)$ are the main products, initiated by the activation-controlled addition of the $\mathrm{H}$ atom to either the $\mathrm{N}$ or the $\mathrm{C}$ atom of $\mathrm{NCN}$. Both theoretical studies agree in that the quartet reaction channel leading to $\mathrm{HNC}$ exhibits a sizeable barrier of about 94 $\mathrm{kJ} \mathrm{mol}^{-1}$ and even at temperatures above $2000 \mathrm{~K}$ contributes only a few percent to the overall reaction.

Rate constant expressions of $k_{2}$ reported in the literature are collected in Table 6 and selected data are included in the Arrhenius plot in Fig. 16. The first theoretical treatment of the reaction $\mathrm{NCN}+\mathrm{H} \rightarrow$ $\mathrm{HCN}+\mathrm{N}(\mathrm{R} 2)$ has been mentioned as a side note in the paper of Moskaleva and Lin [7]. Without giving further details, a first estimate of the rate constant for reaction channel (R2) has been reported in that paper. The reaction has been later reinvestigated by the same group [16] yielding very similar results (blue solid line in Fig. 16). Recently, Klippenstein et al. [17] presented a complete re-assessment of the potential energy surface of the reaction and reported a significantly lower (factor of 2.2 at $2000 \mathrm{~K}$ ) rate constant value for $k_{2}$ (blue dotted line). All studies agree that this reaction channel exhibits an energy 
barrier of about $23-26 \mathrm{~kJ} \mathrm{~mol}^{-1}$, resulting in activation energies of $29-34 \mathrm{~kJ} \mathrm{~mol}^{-1}$ at $T=2000 \mathrm{~K}[14,15]$. Consequently, this channel gains importance toward higher temperatures.

Table 6

Temperature dependence of the rate constant for the reaction $\mathrm{NCN}+\mathrm{H} \rightarrow \mathrm{HCN}+\mathrm{N}$ (R2) expressed in the form $k_{2}=A(T / \mathrm{K})^{n} \exp (-E / R T)$.

\begin{tabular}{|l|l|l|l|l|l|}
\hline$A / \mathrm{cm}^{3} \mathrm{~mol}^{-1} \mathrm{~s}^{-1}$ & $n$ & $E / \mathrm{kJ} \mathrm{mol}^{-1}$ & $T / \mathrm{K}$ & $p / \mathrm{kPa}$ & Refs. \\
\hline $1.89 \times 10^{14}$ & 0.0 & 35.25 & & & Moskaleva and Lin [7] \\
\hline $3.2 \times 10^{13}$ & 0.0 & 0.0 & $2378-2492$ & 50 & Vaseduvan et al. [31] \\
\hline $4.96 \times 10^{12}$ & 0.41 & 22.8 & $298-3000$ & $6-9870$ & Teng et al. [16] \\
\hline $7.94 \times 10^{12}$ & 0.41 & 22.8 & $962-2425$ & $30-210$ & Faßheber et al. [102] $^{\mathrm{a}}$ \\
\hline $3.839 \times 10^{14}$ & 0.0 & 33.29 & & & Lamoureux et al. [52] $^{\mathrm{b}}$ \\
\hline $2.2 \times 10^{11}$ & 0.71 & 22.26 & $500-2500$ & 100 & Klippenstein et al. [17] $^{\mathrm{c}}$ \\
\hline
\end{tabular}

${ }^{a}$ recommended rate constant expression based on $\Delta_{\mathrm{f}} H^{\circ}{ }_{298}(\mathrm{NCN})=450 \mathrm{~kJ} \mathrm{~mol}^{-1}$; the recommendation corresponds to the rate constant expression from Ref. [16] scaled by a factor of 1.6

b equal to 1.1 times the best fit value from Ref. [102]; note that there are misprints in Tables 5 and 6 of Ref. [52], but the expressions given in the Supplementary Material of Ref. [52] are correct

c data in PLOG format for $1 \mathrm{kPa}<p<10 \mathrm{MPa}$ are given in the Suppl. Mat. of Ref. [17]

To date, only two experimental shock tube studies are available for the reaction $\mathrm{NCN}+\mathrm{H} \rightarrow$ products. The first study by Vasudevan et al. [31] yielded five individual rate constant data points for the reaction channel $\mathrm{NCN}+\mathrm{H} \rightarrow \mathrm{HCN}+\mathrm{N}$ (R2) at nominally four different temperatures between 2378 $\mathrm{K}$ and $2492 \mathrm{~K}$ (crosses in Fig. 16). The authors, after having determined the overall rate constant of $\mathrm{CH}$ $+\mathrm{N}_{2}$ and the yield $k_{1 \mathrm{a}} /\left(k_{1 \mathrm{a}}+k_{1 \mathrm{~b}}\right)$, analyzed normalized NCN decay profiles observed at longer reaction times by modeling them with the NCN reaction subset from Ref. [7]. The rate constant $k_{2}$ served as the fit parameter, and values in agreement with those from Refs. [7,16] were obtained, however, with an estimated large uncertainty of about a factor of 2 . Hence, within error limits, these data are also consistent with the lower theoretical rate constant expression given in Ref. [17].

The second shock tube work on the NCN $+\mathrm{H}$ reaction was performed by Faßheber et al. [102]; it provided overall rate constants over a wider temperature range between $962 \mathrm{~K}$ and $2425 \mathrm{~K}$. The thermal decompositions of $\mathrm{NCN}_{3}$ and $\mathrm{C}_{2} \mathrm{H}_{5} \mathrm{I}$ were used for the production of $\mathrm{NCN}$ radicals and $\mathrm{H}$ atoms, respectively, with a large excess of the latter to ensure pseudo-first order reaction conditions. As usual, $\mathrm{NCN}$ has been detected by laser absorption of ${ }^{3} \mathrm{NCN}$ and the initial ${ }^{1} \mathrm{NCN}$ relaxation to ${ }^{3} \mathrm{NCN}$ has been treated as a CIISC process (see Section 5.2). Under the experimental conditions of their work, both the thermal decomposition of $\mathrm{NCN}_{3}$ and the $\mathrm{NCN}$ relaxation were fast compared to the observed ${ }^{3} \mathrm{NCN}$ decay such that the overall NCN rate constant could be determined from the observed NCN profile in a straightforward manner and without interference from initial ${ }^{1} \mathrm{NCN}$ chemistry. The results, in the range 
$(4-9) \times 10^{13} \mathrm{~cm}^{3} \mathrm{~mol}^{-1} \mathrm{~s}^{-1}$, along with a least-squares fit are shown in Fig. 16 as circles and the black long-dashed curve. They reveal a shallow minimum of the overall rate constant at a temperature of about $1100-1200 \mathrm{~K}$ that has been interpreted by the interplay of the $\mathrm{CH}+\mathrm{N}_{2}$ (R-1a) channel predominating at the lower temperatures and the activation-controlled $\mathrm{HCN}+\mathrm{N}(\mathrm{R} 2)$ channel prevailing at the higher temperatures of the experimental range. It is tempting to attribute part of the observed rate constant increase towards higher temperatures to a possible increasing contribution of the ${ }^{1} \mathrm{NCN}+\mathrm{H}$ reaction of thermally equilibrated NCN. Note, however, that even at $T=2500 \mathrm{~K}$, a $10 \%$ singlet contribution would require the singlet rate constant to be about 40 times higher than for the triplet reaction (see Fig. 13). Hence, assuming a triplet rate constant of $5 \times 10^{13} \mathrm{~cm}^{3} \mathrm{~mol}^{-1} \mathrm{~s}^{-1}$, this would imply a singlet rate constant value of $2 \times 10^{15} \mathrm{~cm}^{3} \mathrm{~mol}^{-1} \mathrm{~s}^{-1}$, which is well above the collision number. Consequently, with a maximum possible contribution of the singlet reaction of about a few percent even at the highest experimental temperature, the determined rate constants can be safely assumed to solely represent triplet reactivity. The grey shaded area in Fig. 16 highlights the reported uncertainty range $\leq 30 \%$ of the rate constant determination [102], mainly reflecting the uncertainty of the assumed overall $\mathrm{H}$ atom yield from the two-channel ethyl iodide decomposition, which had been adopted from the most recent global optimization study of Varga et al. [138], and the possible influence of an interfering NCN loss by the reaction $\mathrm{NCN}+\mathrm{N}$. No pressure dependence has been found over the experimental pressure range from 28 to $213 \mathrm{kPa}$ [102]. The authors discuss a value of $\Delta_{\mathrm{f}} H^{\circ}{ }_{298}(\mathrm{NCN})=450 \mathrm{~kJ} \mathrm{~mol}^{-1}$ to be most consistent with their rate data and estimate a robust upper limit of $\Delta_{\mathrm{f}} H^{\circ}{ }_{298}(\mathrm{NCN})<456 \mathrm{~kJ} \mathrm{~mol}^{-1}$. The reported rate constant expressions for channels (R-1a) and (R2) are included in Table 5 and Table 6, respectively.

Fig. 16 compares the overall rate constant of Faßheber et al. [102] with modeling and theoretical results. The black dot-dashed curve is based on the modeling of prompt-NO formation in low-pressure flames by Lamoureux et al. [50] as it is described in more detail in Section 7.2.1. The rate constant $k_{2}$ was adjusted after considering the experimental results of Ref. [102] and by adopting the rate constant $k_{3}$ from Teng et al. [16]. As such, it is largely consistent with the experimental result for the overall rate constant. Also the most recent theoretical estimate of the overall rate constant (black short-dashed curve) from Klippenstein et al. [17] is in reasonable agreement with the experimental results from Faßheber et al. [102] within $\pm 50 \%$. However, the global temperature dependence and in particular the temperature of the rate constant minimum is not well captured. This has substantial consequences for the prompt-NO switch temperature, which will be analyzed in more detail in Section 8 . 


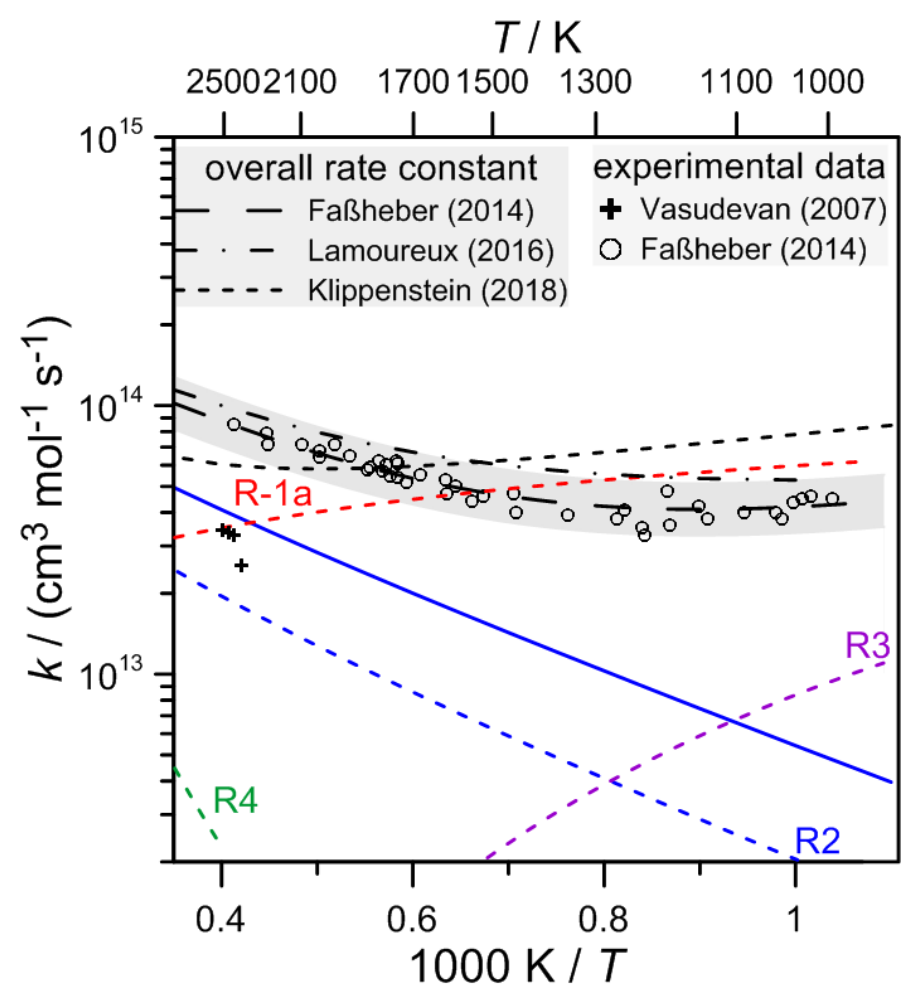

Fig. 16. Arrhenius plot of selected rate constants for the reaction $\mathrm{NCN}+\mathrm{H} \rightarrow$ products. Comparison of results from experiments [31,102] and theoretical calculations at $p=100 \mathrm{kPa}[16,17]$. The data by Vasudevan et al. [31] (crosses) and Teng et al. [16] (solid blue curve) correspond to the reaction channel $\mathrm{NCN}+\mathrm{H} \rightarrow \mathrm{HCN}+\mathrm{N}(\mathrm{R} 2)$, whereas the data and uncertainty limit from Faßheber et al. [102] (circles, grey shaded area) refer to the overall rate constant. The overall rate constant from GDFkin ${ }^{\circledR} 3.0$ NOMecha2.0 model [52] is shown as dot-dashed curve. The rate constant expressions calculated by Klippenstein et al. [17] for each of the four channels yielding $\mathrm{CH}+$ $\mathrm{N}_{2}$ (R-1a), HCN + N (R2), HNCN (R3) and $\mathrm{HNC}+\mathrm{N}(\mathrm{R} 4)$ are shown as colored and the resulting overall rate constant as black dotted curves.

The calculated rate constants of the four individual reaction channels as reported by Klippenstein et al. [17] are included in Fig. 16 as colored dotted curves as well. This theoretical study, in agreement with the results of Harding et al. [15] and Teng et al. [16] (not shown in Fig. 16 for the sake of clarity), predicts that at intermediate pressures $\mathrm{CH}+\mathrm{N}_{2}(\mathrm{R}-1 \mathrm{a})$ is the dominant product channel at typical flame temperatures (red dotted curve). However, the experimentally observed increase of the overall rate constant at temperatures above $1500 \mathrm{~K}$ indicates that an activation-controlled reaction may significantly contribute to the overall rate constant already at much lower temperatures than predicted in Ref. [17]. In this light, the theoretically predicted rate constants of the reaction channels (R2) (blue dotted curve) and/or (R4) (green dotted curve) may be too low. In order to clarify this remaining discrepancy, Klippenstein et al. [17] also investigated potential additional contributions of channel (R4) resulting from ISC from ${ }^{2} \mathrm{HNCN}$ to ${ }^{4} \mathrm{HNCN}$ by a sophisticated non-adiabatic transition state theory (NST) approach [139]. Although ISC was found to significantly contribute to the $\mathrm{HNC}+\mathrm{N}$ channel by bypassing its high entrance barrier, the NST treatment did not change the overall picture very much. 
Finally, theory predicts that at lower temperatures and higher pressures the $\mathrm{HNCN}$-forming combination reaction (R3) may contribute as well [17]. This reaction channel exhibits a pronounced pressure dependence and a distinct negative temperature dependence. As it is reflected by the purple dotted curve in the Arrhenius plot in Fig. 16, at atmospheric pressure and temperatures above $1000 \mathrm{~K}$, this reaction channel plays a minor role, but in high-pressure flames at $p=10 \mathrm{MPa}$ collisional stabilization of HNCN may dominate the overall reaction up to about $1500 \mathrm{~K}$ [17]. To the best of our knowledge, no experimental data are available on this reaction channel so far.

Clearly, more experimental work on the kinetics of the important $\mathrm{NCN}+\mathrm{H}$ reaction is highly desirable. For example, monitoring the yield of HCN (and HNC) in experiments similar to those performed by Faßheber et al. [102] could offer a way to further assess and resolve the issue of the hightemperature branching ratio and with it narrow down the transition temperature of the prompt-NO switch (see Section 8).

\subsection{Other NCN-consuming reactions}

Next to the key role of the reaction $\mathrm{NCN}+\mathrm{H}$ for the prompt-NO switch (see Section 8), a full mechanistic description of prompt-NO formation needs to account for other NCN-consuming reactions as well. The products of these reactions open new or contribute to alternative $\mathrm{NO}_{\mathrm{x}}$ formation pathways aside from the Fenimore route $\mathrm{CH}+\mathrm{N}_{2} \rightarrow \mathrm{HCN}+\mathrm{N}$. As it is the case for $\mathrm{NCN}+\mathrm{H}$, many of these reactions are characterized by a complex interplay of pressure-dependent association/recombination reaction channels, typically dominating at low temperatures and high pressures, and activationcontrolled, often direct or indirect abstraction-type reaction channels, prevailing at high temperatures. For such reactions, the rate constants and branching ratios are highly pressure- and temperaturedependent, often with a pronounced minimum of the observed overall rate constant at intermediate temperatures. In order to further elucidate this common behavior, the following compilation of NCNconsuming reactions starts with the very prototypical reactions $\mathrm{NCN}+\mathrm{NO}$ and $\mathrm{NCN}+\mathrm{NO}_{2}$ despite their less important role for practical flame modeling.

\subsection{1 $\mathrm{NCN}+\mathrm{NO} / \mathrm{NO}_{2}$}

The reaction $\mathrm{NCN}+\mathrm{NO}$ has been extensively studied over a wide range of temperatures, $251 \mathrm{~K}<$ $T<1659 \mathrm{~K}$, and pressures, $0.3 \mathrm{kPa}<p<5.0 \mathrm{MPa}[107,109,110,112]$, complemented by theoretical work $[109,110,140,141]$. The very good agreement between theory and experiment within their uncertainty limits underpins the current capabilities to predict and measure NCN rate constants. Fig. 17a illustrates the schematic potential energy diagram for the kinetically most relevant pathways as derived 
by Huang et al. [110] on $\mathrm{G} 2 \mathrm{M}(\mathrm{CC} 5)$ level of theory. The reaction includes both reaction pathways involving different electronic states (here, ${ }^{2} \mathrm{~A}^{\prime}$ and ${ }^{2} \mathrm{~A}^{\prime \prime}$ ) and complementary pathways to either recombination products (trans- or cis-NCNNO, marked as the blue-dotted box in Fig. 17b) or abstraction-type products, in this case from an indirect abstraction reaction forming $\mathrm{CN}+\mathrm{N}_{2} \mathrm{O}$ (marked as the red-dotted box). Recombination takes place via barrierless formation of the trans- and cis$\mathrm{NCNNO}$ intermediate on the energetically less favorable ${ }^{2} \mathrm{~A}$ " electronic surface, followed by internal conversion to the ${ }^{2} \mathrm{~A}^{\prime}$ electronic ground state surface with conical intersections located at energies significantly lower than the entrance energy of NCN + NO. Under such conditions, it is reasonable to assume that internal conversion is not a critical rate-limiting step and can be modeled with RRKM theory assuming a transition probability of $1[109,110]$. The results of the multichannel RRKM/ME simulations by Huang et al. [110] are included in the Arrhenius plot in Fig. 17b as dark yellow curves for two representative pressures of $11 \mathrm{kPa}$ and $53 \mathrm{kPa}$. Due to collisional deactivation of the initially highly excited $\mathrm{NCNNO}^{*}$ adduct, the reaction is strongly pressure-dependent and exhibits the typical negative temperature dependence of a recombination reaction. As the exit barriers prevent dissociation into the products $\mathrm{CN}+\mathrm{N}_{2} \mathrm{O}$, the main product at low and intermediate temperatures is trans-NCNNO. However, with increasing temperature an indirect abstraction pathway forming $\mathrm{CN}+\mathrm{N}_{2} \mathrm{O}$ becomes competitive, resulting in a distinct minimum of the overall rate constant. The rate constant of the indirect abstraction channel is independent of pressure and its positive temperature dependence reflects the involved activation barriers. The outlined mechanistic picture with the pressure-dependent recombination dominating at low to intermediate temperatures and the activation-controlled indirect abstraction dominating at high temperatures is fully supported by experiments. Selected data have been included in the Fig. 17b for comparable total pressures around $50 \mathrm{kPa}$. Static and slow-flow reactor experiments at low to intermediate temperature, performed by Baren and Hershberger [107] and Huang et al. [110], clearly revealed a distinct pressure dependence. The most comprehensive study of the pressure dependence has been performed by Welz and Olzmann [109], covering the pressure range $1.0 \mathrm{kPa}<p$ $<5.0 \mathrm{MPa}$. The fall-off behavior could be very well rationalized by a master equation analysis; a few selected data points and fitted rate constants from their study have been included in Fig. 17b as purple symbols and a curve for a pressure of $50 \mathrm{kPa}$. Moreover, to allow for a comparison with the only available high-temperature shock tube data of Dammeier and Friedrichs [112] (star symbols), the dashdotted purple curve has been calculated from their reported Troe parametrization for a total density of $\rho$ $=2.5 \times 10^{-6} \mathrm{~mol} \mathrm{~cm}{ }^{-3}$. The high-temperature extrapolation is nicely consistent with the onset of the shock tube data that have been performed at total densities from $1.7 \times 10^{-6}$ to $4.3 \times 10^{-6} \mathrm{~mol} \mathrm{~cm}^{-3}(12.3$ $\mathrm{kPa}<p<69.0 \mathrm{kPa})$. 

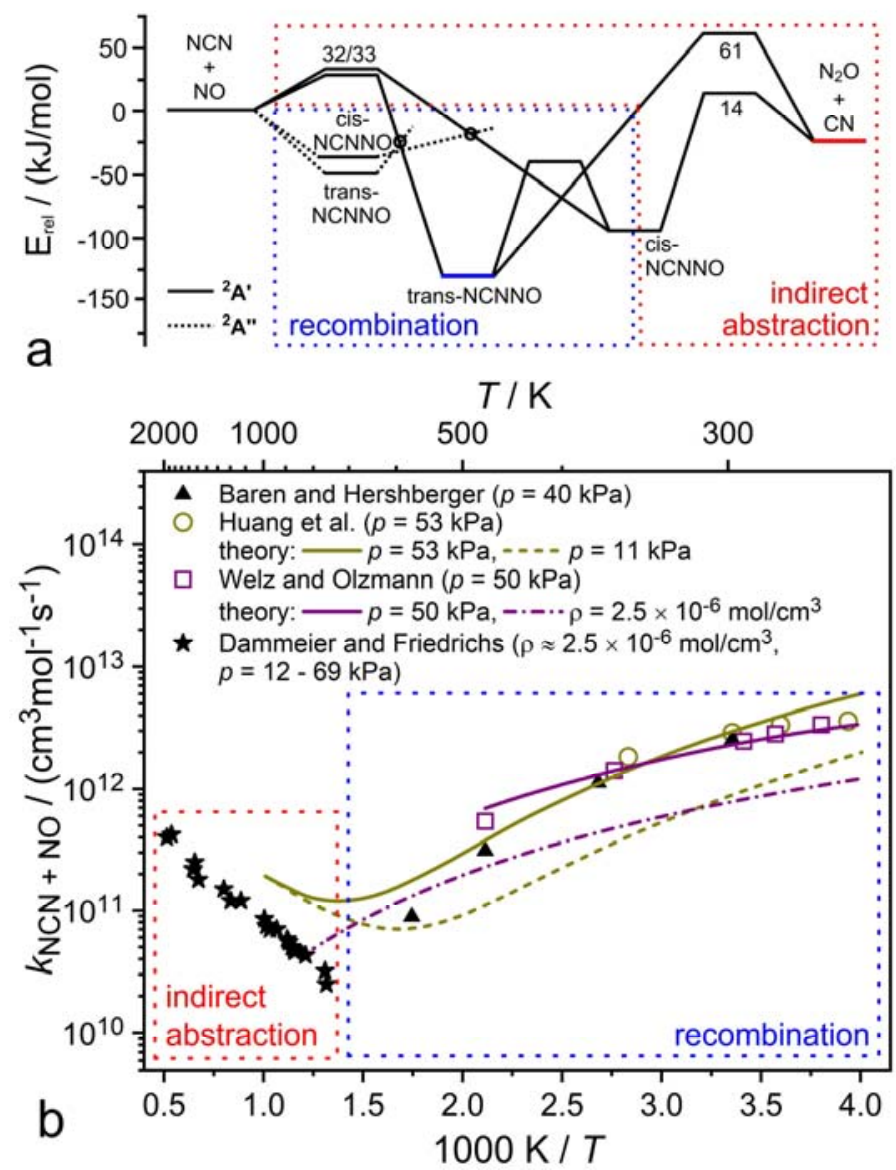

Fig. 17. Reaction NCN + NO - recombination versus indirect abstraction pathway. a) Potential energy diagram, adapted from the G2M(CC5) calculations from Ref. [110], and b) Arrhenius plot of experimental and theoretical data at comparable pressures and densities, taken from Baren and Hershberger [107], Welz and Olzmann [109], Huang et al. [110], and Dammeier and Friedrichs [112].

Calculated potential energy data are also available for the similar reaction NCN + NS, where the NS radical is discussed to be an important intermediate combining nitrogen and sulfur chemistry, see Ref. [140].

The reaction $\mathrm{NCN}+\mathrm{NO}_{2}$, which has been studied both experimentally and theoretically, is another prime example of an elementary reaction with association-dominated kinetics at low and abstractiondominated kinetics at high temperature. Following the early experiment of Baren and Hershberger [107], who only reported an upper limit for the room temperature rate constant, Yang et al. [108] and Kappler [142] investigated the strongly pressure-dependent reaction by means of 193/248 nm excimer laser photolysis of $\mathrm{NCN}_{3}$ combined with laser-induced fluorescence detection of $\mathrm{NCN}$, covering a wide pressure range of $15 \mathrm{kPa}<p<3.8 \mathrm{MPa}$ at overall low temperatures of $255 \mathrm{~K}<T<349 \mathrm{~K}$. The somewhat higher rate constants reported by Yang et al. [108] are most likely due to interfering NCN loss from the reaction $\mathrm{NCN}+\mathrm{O}$ with $\mathrm{O}$ atoms stemming from $\mathrm{NO}_{2}$ photolysis. Nevertheless, both the consistently reported positive pressure and the negative temperature dependencies of the rate constant are in agreement with an association reaction forming the recombination product $\mathrm{NCN}-\mathrm{NO}_{2}$. At the highest 
pressures used in the study of Kappler [142], the reaction proceeds close to its high-pressure limit, hence a reliable high-pressure rate constant extrapolation was possible $\left(k_{\infty}(298 \mathrm{~K})=4.4 \times 10^{11} \mathrm{~cm}^{3} \mathrm{~mol}^{-1} \mathrm{~s}^{-1}\right)$ and the fall-off behavior could be accurately modeled. Quantum chemical and statistical rate theory calculations were performed with energies and structures obtained on the G2M(CC5) level of theory [108], showing that potential decomposition channels of $\mathrm{NCN}-\mathrm{NO}_{2}$ all exhibit energy barriers higher than the $\mathrm{NCN}+\mathrm{NO}_{2}$ entrance energy. Even at higher temperatures, these decomposition channels are not important because an alternative direct abstraction pathway forming NCNO + NO prevails. The corresponding switch from a negative to a positive temperature dependence of the rate constant stemming from the about $40 \mathrm{~kJ} \mathrm{~mol}^{-1}$ energy barrier of the abstraction reaction and the expected vanishing of the pressure dependence has been confirmed by the high-temperature shock tube study of Dammeier and Friedrichs [112]. Standard Arrhenius behaviour has been observed in their experiments at $704 \mathrm{~K}<T<1659 \mathrm{~K}$ and $18.2 \mathrm{kPa}<p<65.4 \mathrm{kPa}$, hence putting the expected, not yet experimentally confirmed overall minimum of the overall rate constant to a temperature of about $500 \mathrm{~K}$. Clearly, at typical combustion temperatures and pressures, the reaction can be safely assumed to yield solely the products $\mathrm{NCNO}+\mathrm{NO}$.

\subsection{2 $N C N+M$}

A first theoretical study dealing with the unimolecular decomposition of NCN was published by Moskaleva and Lin in 2001 [53]. It was shown that ground state $\mathrm{NCN}\left({ }^{3} \Sigma_{\mathrm{g}}^{-}\right)$isomerizes via a cyclic intermediate (c-NCN) to $\mathrm{CNN}$, which decomposes mainly toward $\left.\mathrm{C}^{3} \mathrm{P}\right)+\mathrm{N}_{2}$. Multichannel RRKM calculations were performed on the basis of molecular data from density functional theory and energies from G2M (RCC) calculations. Rate expressions for the different reaction channels were derived (note that there are misprints in Table 7 of Ref. [53], see Ref. [18] of Ref. [116]).

So far there are two experimental studies available on the thermal decomposition of NCN $[111,116]$. In both works, shock tube techniques were used with $\mathrm{NCN}_{3}$ as precursor for $\mathrm{NCN}$ and with $\mathrm{Ar}$ as bath gas. Whereas Dammeier et al. [111] monitored the NCN decay with narrow-bandwidth laser absorption, Busch et al. [116] monitored the $\mathrm{C}$ atom production with atomic resonance absorption spectroscopy. Despite the different detection methods and the different species monitored, the results of these works are in very good agreement. The reaction was shown under shock tube conditions to be in the lowpressure second-order regime at pressures between 100 and $400 \mathrm{kPa}$, which is in agreement with the predictions from Ref. [53].

In Ref. [116] also a master equation modeling was performed on the basis of molecular data and energies calculated at the $\mathrm{CCSD}(\mathrm{T}) / \mathrm{CBS} / / \mathrm{CCSD} / \mathrm{cc}-\mathrm{pVTZ}$ level of theory. A stepladder model obeying detailed balancing was used, and the average energy transferred per down-collision, $\Delta E_{\mathrm{SL}}$, was treated as the adjustable parameter. The fitted value of $\Delta E_{\mathrm{SL}}=1500 \mathrm{~cm}^{-1}$ appears a bit high for NCN collisions 
with the bath gas Ar, but this is often observed for the stepladder model. The second-order rate expression obtained reproduces the experimental results from both experimental works $[111,116]$ within their error margins. Arrhenius parameterizations of the master equation results for $T=1700-3000 \mathrm{~K}$ and $p<5 \mathrm{MPa}$ are available from Table S8 of Ref. [116]. It also turned out from the quantum chemical calculations of Ref. [116] that the rate-limiting step in the decomposition of NCN is probably the c$\mathrm{NCN} \rightarrow \mathrm{CNN}$ isomerization and not the bond fission reaction $\mathrm{CNN} \rightarrow \mathrm{C}+\mathrm{N}_{2}$ as proposed before [53]. The tight transition state for the reaction $\mathrm{CNN} \rightarrow \mathrm{C}+\mathrm{N}_{2}$ found in Ref. [53] was identified in Ref. [116] to be not rate-limiting because an energetically lower-lying pathway directly leading to the products $\mathrm{C}$ $+\mathrm{N}_{2}$ exists.

The overall reverse reaction, $\mathrm{C}+\mathrm{N}_{2} \rightarrow$ products, was also modeled by Moskaleva and Lin [53]. The calculated overall rate constant in the temperature range $T=2500-4500 \mathrm{~K}$ at a pressure of $p=100 \mathrm{kPa}$ agrees very well with experimental values obtained in two earlier shock tube studies $[32,134]$ though a somewhat higher activation energy is predicted. The by far dominant product channel at these high temperatures was calculated to be $\mathrm{N}+\mathrm{CN}$. A third-order rate constant of the $\mathrm{C}+\mathrm{N}_{2}+\mathrm{M}$ reaction at room temperature and pressures of $p=20-70 \mathrm{kPa}$ with $\mathrm{Ar}$ as bath gas was experimentally determined by Husain and Kirsch [143]. These workers assumed recombination to be the dominating channel under these conditions, a fact that was strongly supported by the calculations of Ref. [116], where a direct recombination path leading to $\mathrm{CNN}$ via a small submerged reef but without a tight transition state was identified. This is confirmed also in two very recent works $[61,62]$.

\subsection{3 $\mathrm{NCN}+\mathrm{H}_{2}$}

The overall rate constant of the reaction with closed-shell molecular hydrogen is expected to be well below the value for collision control. The reaction is of abstraction type, and the formation of the HNCN $+\mathrm{H}$ products is endothermic by about $91 \mathrm{~kJ} \mathrm{~mol}^{-1}$. The associated sizeable activation energy was probably the reason why this reaction had not been included in early flame mechanisms despite the fact that the concentrations of molecular hydrogen in flames can be high, in particular in the flame front of rich hydrocarbon flames. Indeed, in a combined shock tube and flame modeling study by Faßheber et al. [113] it was shown that the reaction can be significant for overall $\mathrm{NO}_{\mathrm{x}}$ formation. With a directly measured rate constant of $1 \times 10^{11} \mathrm{~cm}^{3} \mathrm{~mol}^{-1} \mathrm{~s}^{-1}$ at $T=2000 \mathrm{~K}$, it is about 25 times faster than the reaction $\mathrm{NCN}+\mathrm{O}_{2}$ (see Section 6.2.5). Next to directly contributing to NCN loss, inclusion of the reaction NCN $+\mathrm{H}_{2} \rightarrow \mathrm{HNCN}+\mathrm{H}$ into the GDFkin ${ }^{\circledR} 3.0$ NOMecha2.0 mechanism [52] also made it necessary to account for $\mathrm{HNCN}$ and $\mathrm{HNC}$ secondary chemistry, where the latter species is formed in the subsequent $\mathrm{HNCN}+\mathrm{O} \rightarrow \mathrm{HNC}+\mathrm{NO}$ reaction. Interestingly, the updated model yielded an $8 \%$ increase of the simulated NO in the burned gases of a rich $(\phi=1.5)$ low-pressure $\mathrm{CH}_{4} /$ air flame, which could hardly be ascribed to the $3.5 \% \mathrm{NCN}$ forward flux resulting from the $\mathrm{NCN}+\mathrm{H}_{2}$ reaction alone. A detailed $\mathrm{N}$-flux analysis revealed an important new $\mathrm{NO}_{\mathrm{x}}$ formation pathway through the reaction sequence $\mathrm{HNC} \rightarrow$ 
$\mathrm{HNCO} \rightarrow \mathrm{NH}_{2} \rightarrow \mathrm{NH} \rightarrow \mathrm{NO}$, but in fact, $\mathrm{HCN} / \mathrm{HNC}$ isomerization and not the reaction $\mathrm{NCN}+\mathrm{H}_{2}$ turned out to be the predominant HNC formation step.

The initially reported activation energy of $101 \mathrm{~kJ} \mathrm{~mol}^{-1}$ [113], while keeping the overall rate at combustion temperatures nearly unchanged, has very recently been updated to a somewhat higher value of $124 \mathrm{~kJ} \mathrm{~mol}^{-1}$ [80]. This change was necessary after it had been recognized that toward lower temperatures the experimental data were biased by the corresponding ${ }^{1} \mathrm{NCN}+\mathrm{H}_{2}$ reaction. According to VTST/RRKM calculations based on CASPT2 energies, ${ }^{1} \mathrm{NCN}$ directly inserts into the $\mathrm{H}-\mathrm{H}$ bond with an energy barrier of $74 \mathrm{~kJ} \mathrm{~mol}^{-1}$ and decomposes to form the nearly thermoneutral products $\mathrm{HNCN}+$ $\mathrm{H}$. The alternative channel forming $\mathrm{CN}+\mathrm{NH}_{2}$ is endothermic by $96 \mathrm{~kJ} \mathrm{~mol}^{-1}$ and does only contribute to a minor extent, hence the main reaction products, $\mathrm{HNCN}+\mathrm{H}$, are the same as for the ${ }^{3} \mathrm{NCN}+\mathrm{H}_{2}$ reaction. Allowing for both the ${ }^{1} \mathrm{NCN}$ and the ${ }^{3} \mathrm{NCN}$ reaction, quantitative agreement was found between the experimental data and the theoretical prediction of the pressure-independent rate constant. For practical use in flame models that typically treat NCN as a sum species, Faßheber et al. [80] also reported an effective Arrhenius rate constant expression (assuming thermal equilibrium between ${ }^{1} \mathrm{NCN}$ and ${ }^{3} \mathrm{NCN}$ ) and recommend to implement the overall reaction with the reaction products $\mathrm{HNCN}+\mathrm{H}$.

As already discussed in more detail in Section 5, the ${ }^{1} \mathrm{NCN}+\mathrm{H}_{2}$ reaction serves as a prototypical reaction to assess the potential role of ${ }^{1} \mathrm{NCN}$ chemistry in thermal system. Although the ${ }^{1} \mathrm{NCN}$ insertion reaction turns out to be much faster than the ${ }^{3} \mathrm{NCN}$ abstraction reaction, despite a 140 times higher rate constant of $k=9.6 \times 10^{11} \mathrm{~cm}^{3} \mathrm{~mol}^{-1} \mathrm{~s}^{-1}$ at a temperature of $1500 \mathrm{~K}$ [80], it still proceeds well below the collision limit. This is in contrast to the $\mathrm{H}_{2}$ insertion reactions of other singlet radicals. For example, the reaction ${ }^{1} \mathrm{CH}_{2}+\mathrm{H}_{2}$ has been shown to take place with a rate constant of $k>5 \times 10^{13} \mathrm{~cm}^{3} \mathrm{~mol}^{-1} \mathrm{~s}^{-1}$ from room temperature up to a temperature of $T=2500 \mathrm{~K}[127,144]$. According to Faßheber et al. [80], the reduced reactivity of ${ }^{1} \mathrm{NCN}$ is most probably due to the energy barrier resulting from the necessary rearrangement of the two unpaired electrons of ${ }^{3} \mathrm{NCN}$ that are centered at the two separate $\mathrm{N}$ atoms. In contrast, in singlet radicals like ${ }^{1} \mathrm{NH},{ }^{1} \mathrm{CH}_{2}$, and ${ }^{1} \mathrm{O}$, both antiparallel electrons are located at a single atom, hence ideally pre-configured for the insertion into the single bond of the co-reactant.

\subsection{4 $\mathrm{NCN}+\mathrm{O}$}

Both theoretical calculations by Zhu and Lin [145] and direct experimental measurements by Dammeier at al. [111] agree that the reaction $\mathrm{NCN}+\mathrm{O} \rightarrow \mathrm{CN}+\mathrm{NO}$ is fast with a nearly temperatureindependent and, up to a pressure of $p=10 \mathrm{MPa}$, pressure-independent rate constant on the order of $8 \times$ $10^{13} \mathrm{~cm}^{3} \mathrm{~mol}^{-1} \mathrm{~s}^{-1}$. Activation energies at $T>1500 \mathrm{~K}$ are below $6 \mathrm{~kJ} \mathrm{~mol}^{-1}$. For $1826 \mathrm{~K}<T<2580 \mathrm{~K}$, Dammeier et al. [111] reported the Arrhenius expression $k=9.6 \times 10^{13} \times \exp \left(-5.8 \mathrm{~kJ} \mathrm{~mol}^{-1} / R T\right)$ $\mathrm{cm}^{3} \mathrm{~mol}^{-1} \mathrm{~s}^{-1}( \pm 40 \%)$, and for $200 \mathrm{~K}<T<3000 \mathrm{~K}$, Zhu and Lin [145] the extended Arrhenius expression $k=2.55 \times 10^{13} \times \mathrm{T}^{0.15} \times \exp \left(141 \mathrm{~kJ} \mathrm{~mol}^{-1} / R T\right) \mathrm{cm}^{3} \mathrm{~mol}^{-1} \mathrm{~s}^{-1}( \pm 10 \%)$. This corresponds to merely $5-17 \%$ 
higher theoretical rate constants for the overlapping temperature range of both studies, somewhat increasing to $22 \%$ when extrapolating the experimental expression to $1500 \mathrm{~K}$.

Briefly, the ab initio molecular orbital and transition state theory calculations on the $\mathrm{G} 2 \mathrm{M}(\mathrm{CC} 1)$ level of theory with CASPT3 and MRCI $+\mathrm{Q}$ refinements for barrierless association and dissociation steps have been performed for the singlet and triplet potential energy surfaces. According to adiabatic spin correlation rules, the triplet-triplet collisional complex may be of singlet, triplet and quintet spin character. Although not explicitly mentioned in the work of Zhu and Lin [145], the quintet association may be safely assumed to take place on a repulsive potential energy surface; consequently, this state only needs to be included in the spin statistics for the calculation of the overall association reaction. Both singlet and triplet NCNO association complexes exhibit exit channels to form ${ }^{2} \mathrm{CN}+{ }^{2} \mathrm{NO}$ with energies of -173 and $-159 \mathrm{~kJ} \mathrm{~mol}^{-1}$ relative to the entrance energy. Consequently, the rate of the overall reaction is association-controlled and alternative product channels, ${ }^{4} \mathrm{~N}+\mathrm{NCO}$ and $\mathrm{CO}+\mathrm{N}_{2}$, contribute to less than 1\% [145]. Experimentally, the rate of reaction has been determined behind shock waves with $\mathrm{O}$ atoms produced from the unimolecular decomposition $\mathrm{N}_{2} \mathrm{O}+\mathrm{M} \rightarrow \mathrm{N}_{2}+\mathrm{O}+\mathrm{M}$. To assure fast $\mathrm{O}$ atom production, temperatures of $T>1826 \mathrm{~K}$ were necessary, hence the unimolecular decomposition of NCN competed with the reaction NCN + O. Nevertheless, as both rate constants for the thermal decomposition of $\mathrm{N}_{2} \mathrm{O}$ [146] and $\mathrm{NCN}$ are well known (see Section 6.2.2), also the rate constant for the reaction $\mathrm{NCN}+\mathrm{O}$ could be reliably extracted from the measured $\mathrm{NCN}$ profiles and has been reported with an uncertainty limit of $40 \%$ by Dammeier at al. [111]. Experimental and theoretical rate constant estimates are in agreement within uncertainty limits.

For prompt-NO modeling, under most combustion conditions, the reaction $\mathrm{NCN}+\mathrm{O} \rightarrow \mathrm{CN}+\mathrm{NO}$ turns out to be one of the most important reactions and opens up non-Fenimore reaction routes for $\mathrm{NO}_{\mathrm{x}}$ formation (see Section 7.2.3). Next to directly yielding NO, it also significantly feeds into the cyanide pool, predominantly forming $\mathrm{NCO}$ in the subsequent reactions $\mathrm{CN}+\mathrm{O}_{2} / \mathrm{OH} \rightarrow \mathrm{NCO}+\mathrm{O} / \mathrm{H}$.

\subsection{5 $\mathrm{NCN}+\mathrm{O}_{2}$}

In early attempts to model the NCN prompt-NO formation pathway, a high and temperatureindependent rate constant of $k=1 \times 10^{13} \mathrm{~cm}^{3} \mathrm{~mol}^{-1} \mathrm{~s}^{-1}$ has been estimated for the triplet-triplet reaction $\mathrm{NCN}+\mathrm{O}_{2}$ [47]. Flame modeling studies of El Bakali et al. [33] and Zsély et al. [51] showed that such a high rate constant would make this reaction one of the most important reactions for the fate of NCN in lean and stoichiometric methane/air flames. However, theoretical $\mathrm{G} 2 \mathrm{M}(\mathrm{CC} 1)$ calculations of the potential energy surface by Zhu and Lin in 2005 [147] revealed both high entrance (44-52 $\left.\mathrm{kJ} \mathrm{mol}^{-1}\right)$ and high exit barriers $\left(86-89 \mathrm{~kJ} \mathrm{~mol}^{-1}\right)$ for the formation and subsequent decomposition of the NCN-OO adduct. Note that the reported entrance barrier is in striking contrast to the well-known, more or less barrierless formation of peroxy radicals in recombination reactions with doublet radicals, $\mathrm{R} \cdot+\mathrm{O}_{2} \rightarrow \mathrm{R}$ - 
OO. The sizeable barrier may arise from the loss of resonance energy of the stable triplet $\mathrm{O}_{2}$ biradical [148] and the necessary reorganization of electron density going along with the formation of the unfavorable singlet biradical ${ }^{1} \mathrm{NCN}-\mathrm{OO}$ with well-separated unpaired electrons located at the terminal $\mathrm{N}$ and $\mathrm{O}$ atoms.

The rate-limiting step of the overall reaction is the formation of the reaction products $\mathrm{NCO}+\mathrm{NO}$ and $\mathrm{CNO}+\mathrm{NO}$ from the $\mathrm{NCN}-\mathrm{OO}$ adduct taking place over cyclic transition state structures. In the temperature range $1000-3000 \mathrm{~K}$, with a yield of $83 \%$, NCO formation is the main product channel [147]. Transition state theory calculations suggested a very slow reaction with a four orders of magnitude lower rate constant value of $k=4.4 \times 10^{8} \mathrm{~cm}^{3} \mathrm{~mol}^{-1} \mathrm{~s}^{-1}$ at $T=2000 \mathrm{~K}$ compared to the initial estimate. This predicted very low rate constant, which makes the reaction $\mathrm{NCN}+\mathrm{O}_{2}$ largely unimportant under typical flame conditions, has been more recently experimentally confirmed by Faßheber and Friedrichs [114]. In their shock tube experiments, up to $17 \% \mathrm{O}_{2}$ in the reaction mixture was needed to induce a measurable effect on the NCN concentration-time profiles. At such high oxygen concentrations, the modeling of the shock tube data was not straightforward. For example, it was necessary to account for vibrational relaxation of $\mathrm{O}_{2}$, which caused gradual temperature changes during the experiment. Moreover, as already outlined in more detail in Section 5.1, the shock heating of $\mathrm{NCN}_{3}$ in the presence of $\mathrm{O}_{2}$ revealed an unusual delayed ${ }^{3} \mathrm{NCN}$ formation. It was attributed to an interfering ${ }^{1} \mathrm{NCN}+\mathrm{O}_{2} \rightarrow{ }^{3} \mathrm{NCN}-\mathrm{OO}$ adduct formation, followed by a rate-limiting ISC process resulting in the re-formation of ${ }^{3} \mathrm{NCN}$. Despite these experimental difficulties, it was possible to determine the overall rate constant for the reaction $\mathrm{NCN}+$ $\mathrm{O}_{2}$ with a stated error of about $\pm 57 \%$. The reported Arrhenius activation energy of $97 \mathrm{~kJ} \mathrm{~mol}^{-1}$ is in line with the theoretically predicted temperature dependence but yields about a factor of five higher rate constants as compared to the theoretical prediction of Zhu and Lin [147]. This is still low enough to make this reaction a minor NCN loss path for the purpose of flame modeling. However, from a theoretical point of view and in order to better understand reactivity differences between singlet and triplet NCN radical chemistry, it would be interesting to confirm the postulated activation-controlled formation of the ${ }^{3} \mathrm{NCN}-\mathrm{OO}$ adduct [114] by high-level electronic-structure calculations.

\subsection{6 $\mathrm{NCN}+\mathrm{OH}$}

A critical review of the implementation of the reaction $\mathrm{NCN}+\mathrm{OH}$ into prompt-NO formation mechanisms reveals some inconsistencies. To date, no experimental rate constant has been reported for this difficult to measure cross-reaction of two reactive radicals. An initial estimate of the hightemperature rate constant for the assumed main reaction products $\mathrm{HCN}+\mathrm{NO}, k=5 \times 10^{13} \mathrm{~cm}^{3} \mathrm{~mol}^{-1} \mathrm{~s}^{-}$ ${ }^{1}$ [47], later turned out to be about a factor of about ten too high. The two available high-level theoretical treatments of Zhu et al. [149] and Klippenstein et al. [17] significantly differ in the assumed main reaction products, but reasonably agree on the pressure- and temperature-dependent overall rate constant (within a factor of $2.5-5$ at $1500 \mathrm{~K}<T<2000 \mathrm{~K}$ at $p=100 \mathrm{kPa}$ ). The reaction is another example for 
a typical complex-forming radical-radical reaction (see Section 6.2.1) with a dominating recombination channel with negative temperature dependence at low temperatures and high pressures, $\mathrm{NCN}+\mathrm{OH} \rightarrow$ $\mathrm{NCNOH}$ (channel (a)), and three activation-controlled exit channels on the doublet surface yielding $\mathrm{NCNO}+\mathrm{H}, \mathrm{HCN}+\mathrm{NO}$, and $\mathrm{NCO}+\mathrm{NH}$ (channels (b), (c), and (d), respectively). Another possible reaction pathway on the quartet surface yielding the products $\mathrm{NCOH}+\mathrm{N}$ does not play a role for the overall kinetics [17]. The resulting minimum of the overall rate constant at $p=100 \mathrm{kPa}$ is predicted to be $k \approx 1.4 \times 10^{12} \mathrm{~cm}^{3} \mathrm{~mol}^{-1} \mathrm{~s}^{-1}$ at a temperature of $T=1000 \mathrm{~K}$ [149] and $T=1550 \mathrm{~K}$ [17]. The main differences between the two theoretical studies manifest themselves in the reported high-temperature products: channel (b) in case of Ref. [149], which was based on energies obtained on G2M(CC1) level, and channel (c) and (d) in case of Ref. [17], which was based on more accurate ANL0 energies. According to Klippenstein et al. [17], these discrepancies can be traced back to a systematic uncertainty of the $\mathrm{G} 2 \mathrm{M}(\mathrm{CC} 1)$ energy, a previously unexplored dissociation barrier for the $\mathrm{O}-\mathrm{H}$ bond cleavage channel (b), and a previously unidentified, with $\Delta_{\mathrm{r}} H^{\circ} \approx-2 \mathrm{~kJ} \mathrm{~mol}^{-1}$ nearly thermoneutral reaction path to $\mathrm{NCO}+\mathrm{NH}$ (channel (d)). Summarizing the results of Klippenstein et al. [17], the recombination channel (a) is dominant at room temperatures, the "new" channel (d) becomes important at intermediate temperature, and the $\mathrm{HCN}+\mathrm{NO}$ channel (c) takes over at $T>1600 \mathrm{~K}$. Interestingly, the previously predicted main high-temperature NCNO channel (b), due to the identified reaction barrier, turns out to be unimportant.

Detailed pressure- and temperature-dependent rate constants, which have been adopted for the flame modeling in this work (see Section 7.2), are reported in the Supplement of Ref. [17]. In this context, it is important to note that conclusions drawn from earlier modeling studies about the importance of the $\mathrm{NCN}+\mathrm{OH}$ reaction for prompt-NO formation needs to be regarded with some caution. Whereas early studies overestimated its influence due to too high rate constant estimates, later studies neglected important reaction channels (at the benefit to $\mathrm{HCN}+\mathrm{NO}$ ) $[49,52,150]$ or were (possibly) biased by flawed rate constant expressions resulting from typos and transcription errors [e.g., typo in the temperature exponent for $k(\mathrm{H}+\mathrm{NCNO})$ in Ref. [149] (0.97 instead of -0.97), adopted in Ref. [49]; suspected typos in rate constant expressions for preliminary data reported in Ref. [151], adopted in Table 2 of Ref. [150], typo in the Arrhenius pre-exponential factor for $k(\mathrm{HCN}+\mathrm{NO})$ in Table 5 of Ref. [52], but correct in simulations and in the Supplementary Material].

Thanks to the theoretical work of both Zhu et al. [149] and Klippenstein et al. [17], it can now be safely assumed that the overall rate of the reaction $\mathrm{NCN}+\mathrm{OH}$ is too slow to make this reaction an important pathway for prompt-NO modeling. Nevertheless, an independent experimental verification of the predicted low rate constant value is clearly desirable. In particular at high pressures, the formation of the recombination product $\mathrm{NCNOH}$, which turns out to be thermodynamically stable even at combustion temperatures, may serve as a useful marker for the reaction flux proceeding through the reaction $\mathrm{NCN}+\mathrm{OH}$. 


\subsection{7 $\mathrm{NCN}+\mathrm{CH}_{n},(n=1,2,3)$}

Information about the reactions of NCN with small hydrocarbon radicals is scarce and, to the best of our knowledge, these reactions have not been considered in detailed flame mechanisms for prompt$\mathrm{NO}$ formation as yet. In particular, the reaction with $\mathrm{CH}_{3}$ radicals, which are abundant at relatively high concentration levels in the flame front, deserves attention and in fact turns out to be important in this work (see Section 7.2.3). Note that in rich laminar methane/air flames, high local mole fractions of $\mathrm{CH}_{3}$ of several per mill (hence comparable to the mole fractions of $\mathrm{OH}$ ) are found at the peak position of NCN. Nearly all what is known about the rate constants and product branching fractions of the reactions $\mathrm{NCN}+\mathrm{CH}, \mathrm{CH}_{2}$, and $\mathrm{CH}_{3}$ dates back to early, very comprehensive theoretical work performed in M.C. Lin's group, mainly accomplished in the Ph.D. thesis of Moskaleva [152]. Parts of the results for the reaction $\mathrm{NCN}+\mathrm{CH}_{3}$ can also be found in an extended abstract book published in 2001 [153], and a summary of the rate constant expressions of selected reaction channels have been reported in Table 1 of Ref. [7], including some updates for the reaction $\mathrm{CH}_{2}+\mathrm{NCN}$. Potential energy diagrams of the various reaction paths have been mostly calculated on the G2M(RCC) and B3LYP/6-311G(d,p) level of theory, followed by multichannel canonical variational RRKM/ME calculations at atmospheric pressure and covering the temperature range of $2000-4000 \mathrm{~K}$. All three reactions are fast and proceed with overall rate constants that only weakly depend on temperature, mainly reflecting the temperature dependence of the initiating radical-radical association step. At a temperature of $T=2500 \mathrm{~K}$, the reported overall rate constants are $k\left(\mathrm{NCN}+\mathrm{CH}_{3}\right)=4.0 \times 10^{13} \mathrm{~cm}^{3} \mathrm{~mol}^{-1} \mathrm{~s}^{-1}, k\left(\mathrm{NCN}+\mathrm{CH}_{2}\right)=1.5 \times 10^{14} \mathrm{~cm}^{3} \mathrm{~mol}^{-1} \mathrm{~s}^{-1}$, and $k(\mathrm{NCN}+\mathrm{CH})=1.2 \times 10^{14} \mathrm{~cm}^{3} \mathrm{~mol}^{-1} \mathrm{~s}^{-1}$.

The reaction ${ }^{3} \mathrm{NCN}+{ }^{2} \mathrm{CH}_{3}$ can proceed on the doublet and quartet potential energy surfaces. On the quartet surface, the $\mathrm{CH}_{3}$ addition to the $\mathrm{C}$ atom of $\mathrm{NCN}$ takes place over a sizeable barrier of $39 \mathrm{~kJ} \mathrm{~mol}^{-}$ 1. This reaction channel yields the exothermic products ${ }^{1} \mathrm{CH}_{3} \mathrm{CN}+{ }^{4} \mathrm{~N}$, but except at very high temperatures (about $3 \%$ branching fraction at $4000 \mathrm{~K}$ ) cannot compete with the barrierless addition of $\mathrm{CH}_{3}$ to the $\mathrm{N}$ atom of $\mathrm{NCN}$ on the doublet surface. Here, at $2500 \mathrm{~K}$ and $100 \mathrm{kPa}$, the direct recombination product ${ }^{2} \mathrm{CH}_{3} \mathrm{NCN}$ is collisionally stabilized to a small extent (14\%), whereas the main reaction products ${ }^{1} \mathrm{CH}_{2} \mathrm{NH}$ and ${ }^{2} \mathrm{CN}$ (84\%) can be formed over an energetically low-lying exit barrier. Note that in Ref. [7], the stated Arrhenius expression for this channel yields much lower rate constants than the Arrhenius expression reported in the original Ref. [153]. The accordingly, a factor of about 100 too low reported overall rate constant at $2500 \mathrm{~K}$ in Ref. [7], next to the difficulties to account for subsequent $\mathrm{CH}_{2} \mathrm{NH}$ chemistry, may be one of the reasons why the reaction $\mathrm{NCN}+\mathrm{CH}_{3}$ has not yet been considered in detailed flame modeling mechanisms. In fact, its high rate constant value has very recently confirmed in yet unpublished preliminary experimental results of Hesse et al. [154]. In shock tube experiments using $\mathrm{NCN}_{3}$ and tert-butylhydroperoxide as precursors for $\mathrm{NCN}, \mathrm{OH}$ and $\mathrm{CH}_{3}\left(\left(\mathrm{CH}_{3}\right)_{3} \mathrm{OOH} \rightarrow\right.$ acetone $+\mathrm{CH}_{3}+\mathrm{OH}$ [155]), the observed $\mathrm{NCN}$ decay profile, due to the very slow $\mathrm{NCN}+\mathrm{OH}$ reaction (see Section 6.2.6), could be attributed almost exclusively to the reaction $\mathrm{NCN}+\mathrm{CH}_{3}$. With $k(T=2000 \mathrm{~K})$ 
$=5.8 \times 10^{13} \mathrm{~cm}^{3} \mathrm{~mol}^{-1} \mathrm{~s}^{-1}$ and a slightly negative temperature dependence, the obtained overall rate constant is largely consistent with the originally reported theoretical prediction of Moskaleva and Lin [153].

The reaction ${ }^{3} \mathrm{NCN}+{ }^{3} \mathrm{CH}_{2}$ can proceed on the singlet, triplet and quintet potential energy surfaces. According to Moskaleva [152], the reaction is initiated by radical-radical association on all surfaces, where ${ }^{1} \mathrm{CH}_{2} \mathrm{NCN}$ on the singlet surface turned out to be the most exothermic intermediate. However, energetically low-lying exit barriers prevent the collisional stabilization of this and of other intermediates. At $T=2500 \mathrm{~K}$ and $p=100 \mathrm{kPa}$, the formation of ${ }^{2} \mathrm{CH}_{2} \mathrm{CN}+{ }^{4} \mathrm{~N}$ on the triplet surface is predicted to be the main reaction channel (70\%), but the isomeric isocyano product ${ }^{2} \mathrm{CH}_{2} \mathrm{NC}+{ }^{4} \mathrm{~N}(8 \%)$ and ${ }^{2} \mathrm{CH}_{2} \mathrm{~N}+{ }^{2} \mathrm{CN}(22 \%)$, the latter on the singlet surface, contribute to the product spectrum as well. Note that a possible contribution from the quintet surface that also correlates with the ${ }^{2} \mathrm{CH}_{2} \mathrm{CH}+{ }^{4} \mathrm{~N}$ reaction channel, have not been considered in Ref. [152], and that additional multi-reference and nonadiabatic calculations may be needed to properly account for the involved high spin states and potential ISC pathways. Therefore, facing the uncertainties of the reported branching fractions, it may well be acceptable to include the fast reaction $\mathrm{NCN}+\mathrm{CH}_{2}$ by assuming the sole products $\mathrm{CH}_{2} \mathrm{CN}+\mathrm{N}$ in future prompt-NO modeling mechanisms.

Finally, Moskaleva [152] considered the reaction ${ }^{3} \mathrm{NCN}+{ }^{2} \mathrm{CH}$ to proceed both on the doublet and on the quartet surface. Following addition to the $\mathrm{N}$ atom of $\mathrm{NCN}$, the intermediates ${ }^{2} \mathrm{NCNCH}$ and (electronically excited) ${ }^{4} \mathrm{NCNCH}$ rearrange and quantitatively decompose to form ${ }^{1} \mathrm{HCN}+{ }^{2} \mathrm{CN}$ on the doublet and ${ }^{3} \mathrm{HCCN}+{ }^{4} \mathrm{~N}$ on the quartet surface. The reaction pathways to both exothermic product sets exhibit low-lying exit barriers, hence no intermediates are stabilized at a pressure of $p=100 \mathrm{kPa}$. At $T=$ $2500 \mathrm{~K}$, the predicted channel branching fractions of $68 \%$ for $\mathrm{HCCN}+\mathrm{N}$ and $32 \%$ for $\mathrm{HCN}+\mathrm{CN}$ largely reflects the spin statistics of the formation of the initial collision complex.

In summary, it can be concluded that the reactions $\mathrm{NCN}+\mathrm{CH}_{\mathrm{x}}$ are fast and therefore should be considered in prompt-NO flame mechanisms. However, the possible branching into several reaction channels that fuel the $\mathrm{N}$ atom or $\mathrm{CN}$ radical pools adds complexity. Therefore, both high-level theoretical calculations and experimental measurements are desirable to better constrain the as yet unexplored roles of these reactions.

\subsection{8 $N C N+C / N / N C N / C N$}

The significance of these presumably fast atom-radical or radical-radical reactions largely stems from their roles under typical reaction conditions as they have been used for NCN rate constant measurements behind shock waves. For example, at not too high temperatures, the decay of NCN radicals in a typical $\mathrm{NCN}_{3}$ thermal decomposition experiment is initiated by the self-reaction of two $\mathrm{NCN}$ radicals. Dammeier et al. [111] reported a rate constant value of $k(\mathrm{NCN}+\mathrm{NCN})=3.7 \times 10^{12} \mathrm{~cm}^{3}$ 
$\mathrm{mol}^{-1} \mathrm{~s}^{-1}$ and postulated the reaction products $2 \mathrm{CN}+\mathrm{N}_{2}$, where the initially formed vibrationally hot $\mathrm{C}_{2} \mathrm{~N}_{2}$ was assumed to instantly dissociate into two $\mathrm{CN}$ radicals. In their study [111], the fitting of the $\mathrm{NCN}$ decay curves were based on a too low NCN absorption cross section (see Section 2.4.2), hence the used NCN radical concentration were probably overestimated and with it the rate constant value underestimated. In the most recent NCN shock tube study of Faßheber et al. [80] a slightly temperaturedependent rate constant, $k=1.1 \times 10^{13} \times \exp \left(-11.7 \mathrm{~kJ} \mathrm{~mol}^{-1} / R T\right.$ ), has been reported (note the misprint of the Arrhenius $A$ factor in Table 1 of Ref. [80]). Hence, based on the updated NCN cross section and detailed kinetic modeling, up to a factor of 1.7 higher rate constants were found to be consistent with the experimental NCN profiles. These higher values are still in agreement with the rough estimate of $k$ $<1.8 \times 10^{13} \mathrm{~cm}^{3} \mathrm{~mol}^{-1} \mathrm{~s}^{-1}$ reported earlier by Benard et al. [122]. This value was deduced from the apparent lifetime of $\mathrm{NCN}$ in a flow reactor experiment where $\mathrm{NCN}_{3}$ was used as a precursor for an electric discharge-induced deposition of a solid carbon nitride film.

The relatively high $\mathrm{CN}$ concentration levels generated in the NCN self-reaction triggers the consecutive $\mathrm{CN}+\mathrm{NCN}$ reaction. Theoretical results for this reaction have been included in Table 1 of Ref. [53] by Moskaleva and Lin but, to the best of our knowledge, details of the calculations have not yet been published. However, at a pressure of $p=0.1 \mathrm{MPa}$, recombination to NCNCN does not seem to be important. Instead, a reaction channel exhibiting an activation energy of $34 \mathrm{~kJ} \mathrm{~mol}^{-1}$ yields $\mathrm{N}$ atoms with rate constant of $k\left(\mathrm{CN}+\mathrm{NCN} \rightarrow \mathrm{C}_{2} \mathrm{~N}_{2}+\mathrm{N}\right)=2.5 \times 10^{12} \mathrm{~cm}^{3} \mathrm{~mol}^{-1} \mathrm{~s}^{-1}$ at $T=2500 \mathrm{~K}$.

The rate constants of the reactions $\mathrm{C} / \mathrm{N}+\mathrm{NCN}$, forming either $2 \mathrm{CN}$ or $\mathrm{N}_{2}+\mathrm{CN}$, have been estimated by He et al. [156] to be $k\left(\mathrm{CN}+\mathrm{NCN} \rightarrow \mathrm{C}_{2} \mathrm{~N}_{2}+\mathrm{N}\right)=1.0 \times 10^{13} \mathrm{~cm}^{3} \mathrm{~mol}^{-1} \mathrm{~s}^{-1}$. Experimentally, Busch [121] was able to narrow down the rate constant of the $\mathrm{C}$ atom reaction to $k(\mathrm{C}+\mathrm{NCN}) \approx 1.0 \times 10^{14}$ $\mathrm{cm}^{3} \mathrm{~mol}^{-1} \mathrm{~s}^{-1}$ by fitting observed $\mathrm{C}$ atom profiles following the thermal decomposition of, $\mathrm{NCN} \rightarrow \mathrm{C}+$ $\mathrm{N}_{2}$, at high temperature. However, it was not possible to clearly separate the contributions from the reactions $\mathrm{C}+\mathrm{NCN}$ and $\mathrm{NCN}+\mathrm{NCN}$, the latter influencing the total $\mathrm{NCN}$ yield.

Although not of key importance for accurate prompt-NO flame modeling, the much limited database on the reactions of $\mathrm{C}, \mathrm{N}, \mathrm{CN}$, with $\mathrm{NCN}$ calls for more experimental and theoretical work. Quantitative detection of the abundant $\mathrm{CN}$ radical may serve as a worthwhile detection target to assess the internal consistency of the NCN reaction system.

\section{NCN as a product of the $\mathrm{CH}+\mathrm{N}_{2}$ reaction in flames: measurement and modeling}

NCN measurements in flames have been accomplished with in situ laser-based diagnostics, both in emission and absorption. Such experiments remain very challenging due to the very weak signal levels and the presence of interfering species close to the NCN excitation transition along the main band head. 
In this section, we present the NCN mole fraction profiles measurements performed using LIF and CRDS. Thanks to these experimental data, it has been possible to assess the robustness of the detailed kinetic mechanisms proposed in the literature. Here, for the sake of brevity, the comparison between the modeling and experimental data is limited to two mechanisms and to the results obtained for rich premixed flames at low and high pressures.

\subsection{Measurements in premixed flames}

\subsubsection{Evidence for NCN in flames}

Smith [38] was the first to verify the existence of $\mathrm{NCN}$ as a product of the $\mathrm{CH}+\mathrm{N}_{2}$ reaction in a 4.0 $\mathrm{kPa} \mathrm{CH} / \mathrm{O}_{2} / \mathrm{N}_{2}$ flame by LIF. NCN radicals were excited at around $329.1 \mathrm{~nm}$ along the main band head of the $\tilde{A}-\tilde{X}$ transitions as recorded from an excitation spectrum. Fluorescence was collected at the same wavelength along the diagonal transitions with a bandwidth of $20 \mathrm{~nm}$. The NCN relative concentration profile measured in this flame peaks in the vicinity of the $\mathrm{CH}$ profile maximum, as it is expected for a product of the reaction between $\mathrm{CH}$ and $\mathrm{N}_{2}$. In addition to the NCN spectral assignment, Smith showed that the LIF signal disappeared when substituting $\mathrm{N}_{2}$ by Ar. Off-diagonal excitation of $\mathrm{NCN}$ along the $\Pi_{\mathrm{u}}(020)-\Sigma_{\mathrm{g}}^{-}(000)$ transition at $316.97 \mathrm{~nm}$ (Fig. 6) and fluorescence collection at $325.9 \mathrm{~nm}$ was also demonstrated [43], but showed spectral interferences with $\mathrm{OH}$ and $\mathrm{CH}$ in a rich $\mathrm{CH}_{4} /$ air flame at $p=$ $0.2 \mathrm{kPa}$. Since Smith's pioneering work, relative concentration profiles of NCN have been measured in several low-pressure premixed flames using the same excitation/collection scheme $[41,42,44,46]$. Evidence for $\mathrm{NCN}$ in atmospheric premixed $\mathrm{CH}_{4} / \mathrm{N}_{2} \mathrm{O} / \mathrm{N}_{2}$ and $\mathrm{CH}_{4} /$ air flames has been demonstrated as well $[43,45]$, but with a very poor signal-to-noise ratio (SNR).

\subsubsection{Absolute quantification of NCN LIF signals}

The quantification of the relative LIF profiles of NCN requires a calibration step in order to convert the relative LIF signal into absolute NCN mole fractions. In a first interesting approach followed by Sutton et al. [41], the NCN LIF intensity was compared with the intensity of a nearby satellite transition of $\mathrm{OH}$ radical, which is more easily related to an absolute concentration value. By considering the spectroscopic parameters of $\mathrm{OH}$ and $\mathrm{NCN}$ and by comparing the LIF intensities of both species, an estimation of the NCN concentration could be obtained. Unfortunately, the attribution of the most intense feature of the LIF excitation spectrum to the sole band head of the $(000)-(000)$ transition led to a misinterpretation of the results in Refs. $[38,41]$ (see also Section 2.3 and Fig. 8). The second reported approach for quantification of NCN was based on directly measuring NCN concentration in a single suitable flame, which then serves as a reference for calibrating the LIF profiles obtained in various other flames. This approach was followed by Lamoureux et al. $[42,44,68]$ by a combination of LIF and CRDS 
techniques. While LIF is a highly sensitive technique with high spatial resolution, CRDS is a line-ofsight technique that allows one to quantify the absorbance across the flame diameter with very high sensitivity.

The absorbance per unit of length of NCN was found to be very low, around $10^{-5} \mathrm{~cm}^{-1}$ only. Thus, a technique as sensitive as CRDS is required to measure NCN in absorption (see e.g. Refs. [157-159]). The principle of pulsed CRDS is the injection of a laser pulse into a resonant optical cavity consisting of two highly reflective mirrors. In this cavity, the laser pulse reflects back and forth and interacts with the absorbing medium, i.e. the flat flame, thousands of times. The main advantages of this technique are firstly its intrinsic insensitivity to light source intensity fluctuations, and secondly the very long effective path length inside the cavity. The reported detection limit of in situ CRDS detection in flames is about $10^{-6} \mathrm{~cm}^{-1}$ [160]. In practice, the laser is alternately tuned off-resonance and on-resonance with the absorption transition. The off-resonance step allows one to measure the decay time of the laser pulse in the cavity in absence of absorption. In this case, the decay is essentially determined by the losses experienced by the laser pulse upon each successive mirror reflection (losses/pass). When the laser is tuned on-resonance, additional losses due to the absorption in the flame lead to a decrease of the decay time. The net absorbance can be obtained from the difference of the decay constants measured on- and off-resonance. Pulsed CRDS has been successfully applied for the detection of several species in flames involved in NO chemistry such as $\mathrm{CH}, \mathrm{CN}, \mathrm{NH}$ and NCO [161-164]. In case of NCN, this technique actually turned out to be more complex because of the compactness of the NCN spectrum and the absence of individual and isolated absorption lines as shown in Fig. 7. In addition, the measured decay times had to be corrected for the significant variation of the cavity performance when scanning the laser over a large spectral range, as it was required to move the detection wavelength sufficiently far away ( $\lambda$ $=330.1 \mathrm{~nm})$ from the NCN band head $(\lambda=329.13 \mathrm{~nm})$ in order to obtain a reliable baseline. For a more detailed description of the complete calibration procedure, we refer to Refs. [44,68].

The first measurements of NCN using CRDS were performed in a rich $\mathrm{CH}_{4} / \mathrm{O}_{2} / \mathrm{N}_{2}$ flame at $5.3 \mathrm{kPa}$. From the on/off procedure, a rather low SNR of around 2-3 was achieved. In order to increase the SNR, several flames of different compositions were tested. Adding nitrogen-containing species like $\mathrm{N}_{2} \mathrm{O}$ significantly increases the NCN signal $[38,45]$, but at the expense of interfering absorption from other species. In particular, the oxidation of the added nitrogen-containing species leads to the formation of large quantities of NH radicals. NH absorption lines interfere with the NCN spectrum as shown in Fig. 18, thus preventing accurate NCN absorbance measurements [165]. The best compromise up to now was found in an acetylene low-pressure flame [44] where the SNR was as high as 6.

A portion of the NCN absorption spectrum around its most intense band head is shown in Fig. 8. Despite the very low SNR, one can indubitably recognize the features due to the most intense band head of NCN. In fact, in these experiments the measured loss/pass at on- and off-resonance wavelengths were very close to each other, as shown in Fig. 19. Since the decay rate was affected by additional non- 
resonant losses along the flame axis, the net absorbance due to the NCN absorption had to be determined by subtraction of the two black and red colored profiles in Fig. 19. The difference signal peaks at merely $4.5 \times 10^{-5}$ for a $6 \mathrm{~cm}$ flame path. Note that a very good agreement between the NCN absorbance profile (open blue circles) and the relative mole fraction profile measured by LIF (black crosses) was obtained. Knowing the absorption cross section as determined in Section 2.4, it was hence possible to convert the measured absorbance into a NCN mole fraction, yielding $326 \mathrm{ppb} \mathrm{NCN}$ for the investigated rich acetylene $/ \mathrm{O}_{2} / \mathrm{N}_{2}$ flame [68]. This flame served as the reference flame for calibrating the relative $\mathrm{NCN}$ profiles obtained by LIF in several other low-pressure flames [68]. For example, the NCN peak mole fraction in a rich methane $/ \mathrm{O}_{2} / \mathrm{N}_{2}$ was determined to be equal to $175 \mathrm{ppb}$ in Ref. [68]. Note, however, that due the very weak absorbance of NCN radicals in the flames, the concentration measurements exhibit quite substantial uncertainty, estimated to about $\pm 55 \%$ in Refs. $[44,68]$. The variation of the rotational population probed at different flame conditions, using either LIF or CRDS, was found to agree very well [68]. Therefore, the uncertainty of the relative NCN mole fractions reported for different flames is smaller, estimated to be $15 \%$ in Ref. [68].

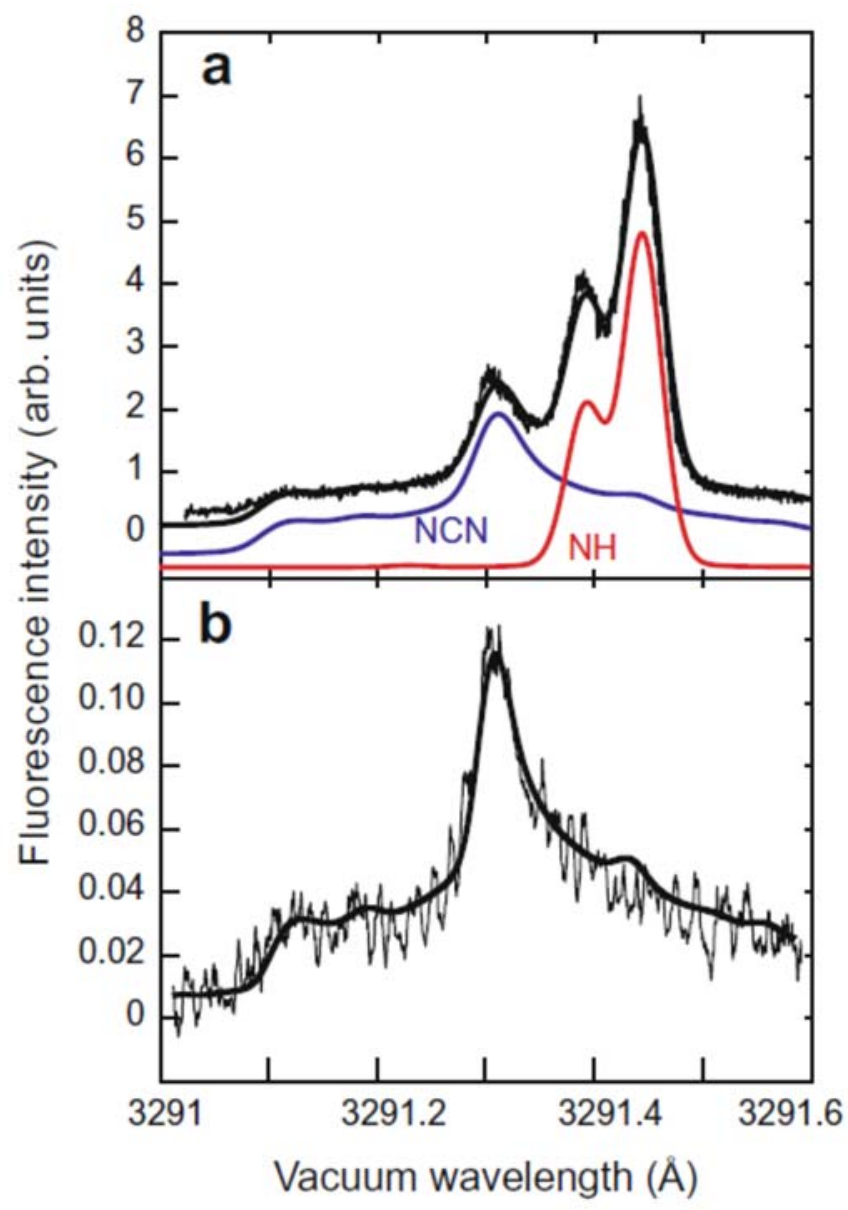

Fig. 18. Comparison of LIF excitation scans performed in atmospheric premixed flames of (a) $\mathrm{CH}_{4} / \mathrm{N}_{2} \mathrm{O} / \mathrm{N}_{2}$ and (b) $\mathrm{CH}_{4} / \mathrm{O}_{2} / \mathrm{N}_{2}$, including simulations by PGOPHER $(T=1900 \mathrm{~K})$. Reprinted from Combustion and Flame, 157, Sun et al., NCN detection in atmospheric flames, 834-836, (C2010, with permission from Elsevier. 


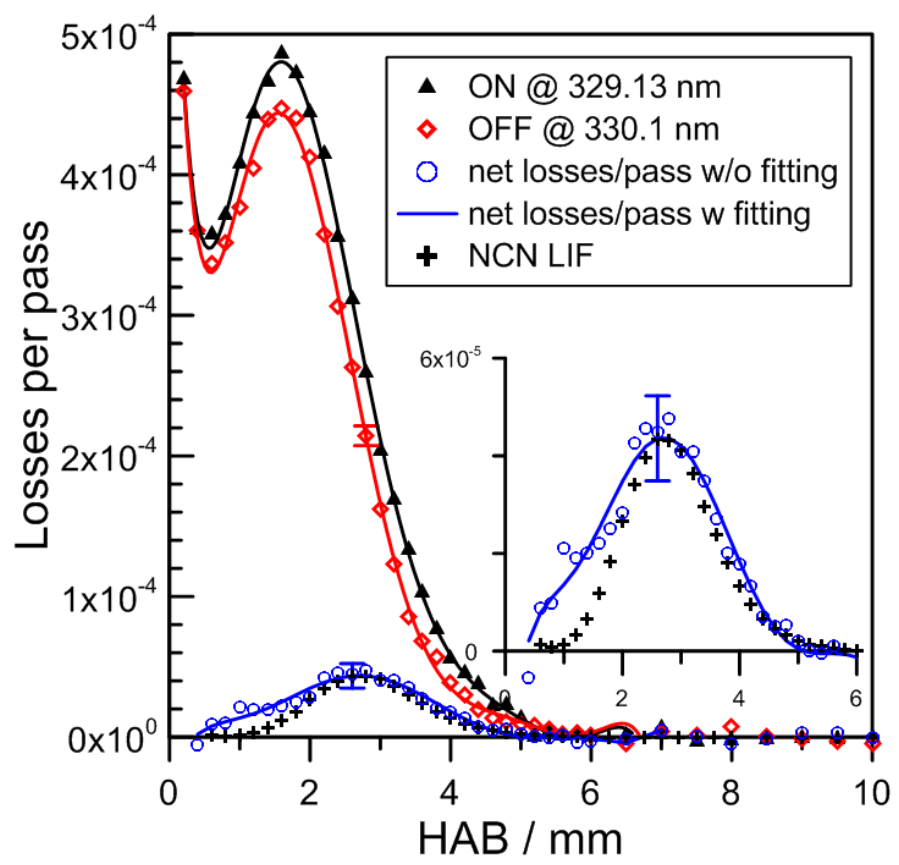

Fig. 19. Illustration of the CRDS results obtained in a rich acetylene flame stabilized at $p=5.3 \mathrm{kPa}$. The insert is a blow-up of the bottom left part of the main figure. The blue curves and symbols represent the best-fit NCN profiles. Adapted from Lamoureux et al. [44].

\subsection{Implementation of the reaction $\mathrm{CH}+\mathrm{N}_{2} \rightleftharpoons \mathrm{NCN}+\mathrm{H}$ in nitrogen chemistry modeling}

The first implementation of NCN chemistry into a detailed kinetic mechanism has to be put to the credit of Glarborg et al. [47] as early as 1998. However, reactions rate constants for the NCN oxidation $\left(\mathrm{NCN}+\mathrm{O}, \mathrm{NCN}+\mathrm{OH}, \mathrm{NCN}+\mathrm{O}_{2}\right)$ and $\mathrm{NCN}$ reduction $(\mathrm{NCN}+\mathrm{H})$ were estimated with relatively high values in comparison to current recommended values (see Section 6). Those four reactions and their rate constant values were adopted in the first detailed mechanism for $\mathrm{NO}_{\mathrm{x}}$ formation explicitly considering the NCN pathway by implementing the reaction $\mathrm{CH}+\mathrm{N}_{2} \rightleftharpoons \mathrm{NCN}+\mathrm{H}$ (R1a) by El Bakali in 2006 [33]. It was validated against prompt-NO measurements in low-pressure flames [166-168]. At that time, the available experimental database consisted of $\mathrm{CH}$ and $\mathrm{NO}$ mole fractions in several low-pressure premixed flames (mostly $\mathrm{CH}_{4} / \mathrm{O}_{2} / \mathrm{N}_{2}$, but also $\mathrm{CH}_{4} / \mathrm{C}_{2} \mathrm{H}_{6} / \mathrm{C}_{3} \mathrm{H}_{8} / \mathrm{O}_{2} / \mathrm{N}_{2}$, from lean to rich equivalence ratio). El Bakali et al. [33] showed that the rate constant value reported in the seminal work of Moskaleva and Lin [7] was definitely too low to reproduce the experimental results. Consequently, the GDFkin ${ }^{\circledR}$ 3.0_NCN mechanism [33] adopted a rate constant for reaction (R1a) determined earlier by Lindackers et al. [134] for the reaction $\mathrm{CH}+\mathrm{N}_{2} \rightarrow \mathrm{HCN}+\mathrm{N}(\mathrm{R} 1 \mathrm{~b})$. It turned out that, due to the very high assumed rate constant value for the reaction $\mathrm{NCN}+\mathrm{H} \rightarrow \mathrm{HCN}+\mathrm{N}(\mathrm{R} 2), \mathrm{NCN}$ radicals seemingly 
are immediately consumed yielding $\mathrm{HCN}$, resulting in a very low mole fraction of $\mathrm{NCN}$. This apparent "invisibility" of the NCN radicals explains why the historical Fenimore pathway (reaction R1b) was still considered in many nitrogen chemistry models for a long time after the initial work of Moskaleva and Lin [7]. However, with the detailed knowledge of NCN chemistry advancing over the years, including the experimental confirmation of much lower rate constants for the reaction $\mathrm{NCN}+\mathrm{H}$, it turned out that this simple conclusion does not hold.

\subsubsection{NCN modeling in low-pressure premixed flames}

As described in Section 7.1.2, NCN radicals profiles have been measured in low-pressure premixed flames by Sutton et al. $[41,46]$ and Lamoureux et al. $[42,44,68,135]$. These experimental studies were combined to improve detailed flame modeling mechanisms.

Sutton et al. $[41,46,150]$ implemented the NCN pathway including the reaction (R1a) with a set of $\mathrm{NCN}$ formation and consumption reactions mostly extracted from theoretical calculations performed by Zhu et al. $[145,147,149]$. This subset was also implemented in three revised mechanisms: GRI-Mech3.0 mechanism [169], GDFkin ${ }^{\circledR} 3.0$ mechanism [33], and USC mechanism [170]. In their later paper, Sutton et al. [46] recommended to adopt the rate constant value of the reaction (R1a) calculated by Harding et al. [15]. For different premixed low-pressure C1-C4 alkane flames, the variation of the NCN profiles was compared to simulations. The experimental increase of $\mathrm{NCN}$ with the number of carbon atoms in the fuel and with the equivalence ratio was well captured by the models for rich, but not for nearly stoichiometric mixtures

Following the initial work of El Bakali et al. [33], the NCN subset reactions have been successively updated and validated up to the final NOMecha2.0 submechanism [52]. It involves 14 reactions involving NCN, for which the rate constant values have mostly been taken from experimental measurements and from high-level theoretical calculations. NOMecha2.0 has already been combined either with the GDFkin ${ }^{\circledR} 3.0$ model to describe alkane oxidation [33] or the acetylene model proposed by Lindstedt and Skevis [171]. NOMecha2.0 was later satisfactorily testedwith the additional measurements of $\mathrm{NH}$ in low-pressure premixed flames [161], and very recently in high-pressure premixed counterflow flames [172]. The mechanism is available as Supplementary Material in Ref. [52]. Note that among the 236 reactions, the values of two pressure-dependent reaction rate constants (HCNO $\rightleftharpoons \mathrm{HCN}+\mathrm{O}$ and $\mathrm{NCN}+\mathrm{H} \rightleftharpoons \mathrm{HNCN}$ ) require appropriate manual selection because the input file is based on old Chemkin-II format.

Very recently, Glarborg et al. [35] proposed a complete revision of the NO formation mechanism where the recent advances in the NCN computational chemistry were adopted. In their model (named KG-model in the following), the NCN subset involves 16 reactions (among them, 11 are common with NOMecha2.0, but only 5 reactions are proposed with the same rate constant values). Thanks to the recent 
high-level theoretical results reported in Ref. [17], they were also able to include pressure-dependent rate constant for the reactions $\mathrm{CH}+\mathrm{N}_{2} \rightleftharpoons \mathrm{NCN}+\mathrm{H}, \mathrm{NCN}+\mathrm{OH} \rightarrow$ products, and $\mathrm{NCN}+\mathrm{H} \rightarrow$ products.

In the following, several modeling results are presented using the KG- and the GDFkin ${ }^{\circledR} 3.0 \_$NOMecha2.0 mechanisms as they were originally published, including the thermochemical data. For clarity, the NCN subsets adopted in these two models are reported in the Supplementary Material (Section C2). During the preparation of the present review, we have realized that the molar heat capacity of $\mathrm{CH}$ in GDFkin ${ }^{\circledR} 3.0 \_$NOMecha2.0 and the one of NCN adopted in the KG-model are both inconsistent with the values reported in the majority of the other references (see Section 3.2 and Fig. 11 for NCN). For consistent use of the published mechanisms, however, this was not changed here but later on for the simulations as presented in Section 8.2.

Tremendous efforts have been devoted during the last decade to better understand the prompt-NO formation through the NCN radical pathway [42,44,52,161-163]. For that purpose, experiments in lowpressure flames have been undertaken for measuring in situ temperature profiles using LIF thermometry and in situ species mole fractions profiles of NO by LIF calibrated by known added amount of NO [44]. NCN $[42,44,68], \mathrm{NCO}$ [163], NH [161] and CN [162] species mole fraction profiles were measured by LIF and calibrated by CRDS at their peak values. HCN species profiles were obtained using continuous wave-CRDS after gas probe sampling [162]. Overall, these measurements form a unique and original experimental database of trace species measurements in $\mathrm{CH}_{4} / \mathrm{O}_{2} / \mathrm{N}_{2}$ and $\mathrm{C}_{2} \mathrm{H}_{2} / \mathrm{O}_{2} / \mathrm{N}_{2}$ flames. Therefore, this dataset has been selected as a suitable validation target for testing the GDFkin ${ }^{\circledR} 3.0 \_$NOMecha2.0 and KG-model performance. As shown in Fig. 20, for the low-pressure rich $\mathrm{CH}_{4} / \mathrm{O}_{2} / \mathrm{N}_{2}$ flame, both models satisfyingly predict the corresponding experimental NCN profiles. A general better agreement between the diverse experimental (symbols) and calculated (curves) species profiles is obtained with GDFkin ${ }^{\circledR}$ 3.0_NOMecha2.0 rather than with KG-model, except for the NCN profiles (in green) for which the simulated peak mole fractions are equal to 101 and $127 \mathrm{ppb}$ with NOMecha2.0 and KG, respectively. Note that the NCN experimental peak target value used here, 175 ppb from Ref. [68], is different from the value used in the recent, similar intercomparison study presented by Glarborg et al. [35]. Accidentally, they used $135 \mathrm{ppb}$, a value reported in Ref. [44], instead of the later reevaluated value of 175 ppb from Ref. [68]. The experimental $\mathrm{CH}$ and $\mathrm{CN}$ profiles (in dark green and light blue) are similarly predicted by the two models and are in good agreement with the experimental profiles. A good prediction of the $\mathrm{CH}$ profile is very important because the $\mathrm{CH}$ radicals are key species of the initiation reaction $\mathrm{CH}+\mathrm{N}_{2} \rightleftharpoons \mathrm{NCN}+\mathrm{H}$ (R1a). However, the other species profiles (NO (red), HCN (black), NCO (blue) and NH (pink)) are poorly predicted with KG-model compared to the good agreement performed by NOMecha2.0. Note, however, that NOMecha2.0 has been optimized using a similar set of validation targets, hence the better agreement of the GDFkin ${ }^{\circledR} 3.0 \_$NOMecha2.0 is not entirely surprising.

As mentioned by Glarborg et al. [35], a better agreement of their simulated NO profile with the experimental one (red curve and symbols in Fig. 20b) could be reached only if the rate constant value 
of the reaction (R2) is increased by a factor of 4 . Such a high rate constant value was not supported by their theoretical computations, but would also improve the agreement with the shock tube data on the total $\mathrm{NCN}+\mathrm{H}$ rate constant reported by Faßheber et al. [102]. Alternatively, Glarborg et al. [35] suggested that the underestimation of the NO yield could be at least partly due to the missing reaction between $\mathrm{NCN}+\mathrm{CH}_{3}$ (introduced in Section 6.2.7), which has not yet been considered in detailed prompt-NO formation mechanisms. We will test this hypothesis in Section 7.2.3.
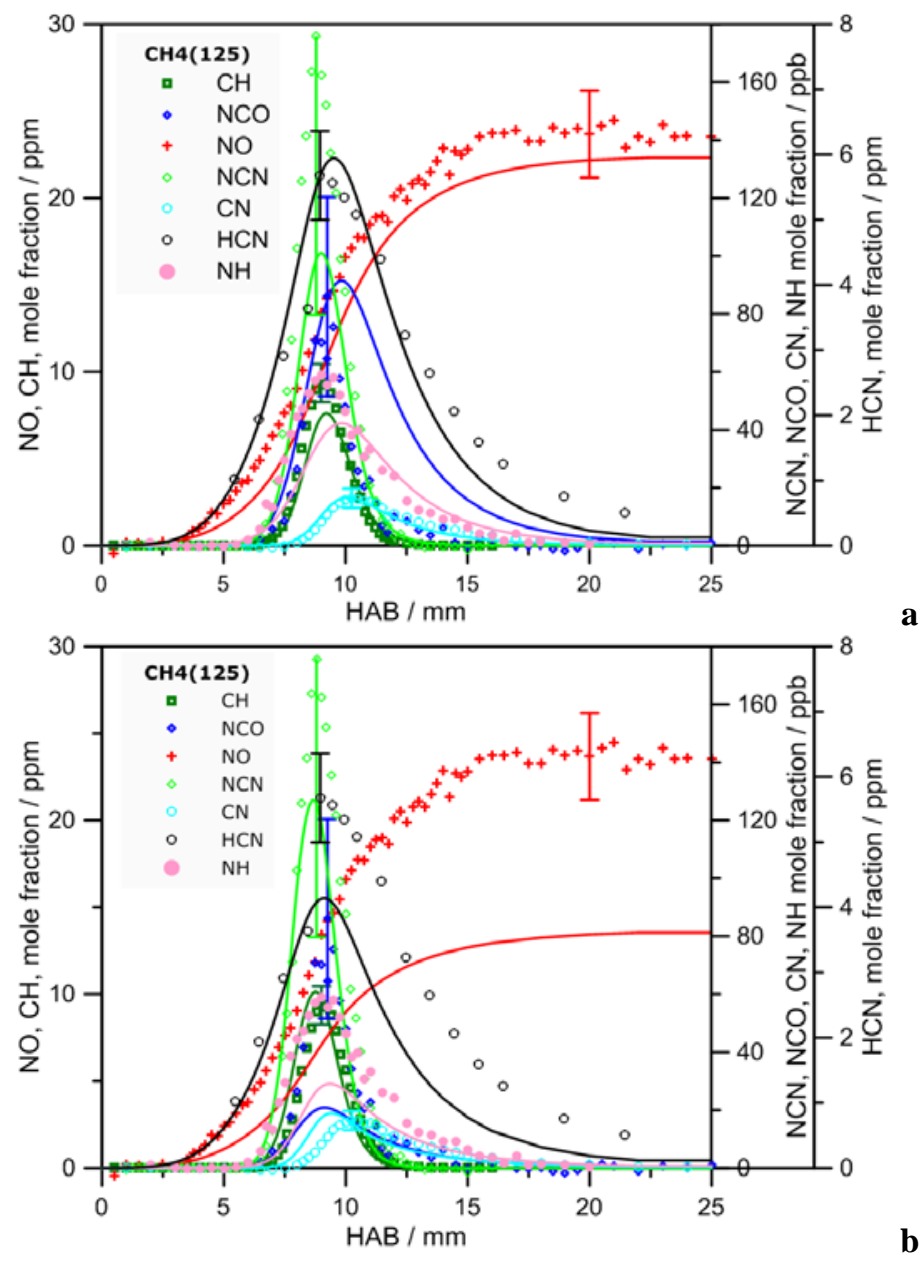

Fig. 20. Comparison between the extended experimental dataset of species profiles (symbols) measured in a rich $\mathrm{CH}_{4} / \mathrm{O}_{2} / \mathrm{N}_{2}$ flame ( $p=5.3 \mathrm{kPa}$ ) with the simulated ones (line, same color as symbols) with a) GDFkin $^{\circledR} 3.0$ NOMecha2.0 [52] and b) KG-model [35]. For clarity, the error bar for NCN is shown in the negative direction only. The unique data set used as the validation target is based on experiments all performed in the same laboratory by Lamoureux et al. $[44,68,135,162,163,172]$ (see text).

\subsubsection{Modeling of NO profiles in rich high-pressure flames}

The availability of NCN profiles in atmospheric and high-pressure flat flames can serve to refine the detailed mechanisms of prompt-NO formation. In an atmospheric burner-stagnation flame, Sun et al. [45] measured the variation of the NCN fluorescence intensity as a function of $\phi$ in the range 0.9-1.6. 
The maximum was observed at $\phi=1.2$ for a $\mathrm{CH}_{4} / \mathrm{O}_{2} / \mathrm{N}_{2}$ flame. Unfortunately, as previously mentioned, NCN profiles have never been measured in absolute concentration in such flames. So, the implementation and verification of the NCN reactions subset on prompt-NO formation can only be evaluated by considering the NO species profiles measured in various flames at atmospheric and supraatmospheric pressures. In the pioneering experiments from the Purdue group [173-175], absolute concentration profiles of $\mathrm{CH}$ and $\mathrm{NO}$ were measured in partially premixed and non premixed highpressure flames by using calibrated LIF. In atmospheric flames, Konnov's group already explored a large range of adiabatic premixed flames of alkanes, alcohols, and ethylene in which the absolute NO mole fraction was measured in the burned gases by using probe sampling techniques $[176,177]$ and LIF [178]. The group from McGill developed a careful experimental LIF procedure for obtaining the absolute mole fraction profiles of $\mathrm{CH}$ and $\mathrm{NO}$ in premixed stagnation flames of $\mathrm{C} 1-\mathrm{C} 4$ alkanes and alcohols [179-181]. More recently the NO measurements were extended to lean supra atmospheric methane/air stagnation flames [182]. In counterflow premixed $\mathrm{CH}_{4} / \mathrm{O}_{2} / \mathrm{N}_{2}$ flames up to $0.7 \mathrm{MPa}$, de Persis et al. [172] measured by LIF the absolute NO mole fraction profiles at three equivalence ratios $(\phi$ $=0.8,1.0$, and 1.2). Such measurements are difficult and rare, and the temperature determination is also challenging. All these data are very useful for testing and improving the prompt-NO mechanisms, but should be also complemented by additional NCN chemistry related species profiles wherever possible.

In order to see the impact of the pressure on the NO formation, in particular the prompt-NO, the works performed in rich flames ( $\phi=1.2)$ from Klassen et al. [173] and from de Persis et al. [172] have been selected to evaluate the performance of the GDFkin3.0HP_NOMecha2.0 [172] and the KG-model [35]. Note that GDFkin3.0HP_NOMecha2.0 denotes the high-pressure (HP) version of GDFkin ${ }^{\circledR} 3.0 \_$NOMecha2.0. This HP version of the GDFkin ${ }^{\circledR} 3.0$ model is directly derived from GDFkin ${ }^{\circledR} 3.0$ initially developed in Ref. [33]. However, in the original version of this mechanism the rate constant values of the pressure-dependent reactions were not clearly indicated, which has been clarified in Ref. [172].

The performance of the pressure-dependent NCN reactions determined by Klippenstein et al. [17] was tested against experimental measurements of NO in high-pressure (from 0.1 to $1.5 \mathrm{MPa}$ ) premixed flames stabilized above a McKenna burner by Klassen et al. [173]. Note that the temperature was only measured in the post-flame. Consequently, the simulated temperature profiles had to be calculated by solving the energy equation assuming an adiabatic flame. In the case of the fuel-rich flames, NO is expected to be predominantly formed through the prompt-NO pathway. The performance of GDFkin3.0HP_NOMecha2.0 and the KG-model with respect to NO measurements performed in rich $\mathrm{CH}_{4} / \mathrm{O}_{2} / \mathrm{N}_{2}$ flames is shown in Fig. 21. Considering the uncertainty due to the absence of experimental temperature profiles, both models predict satisfactorily the evolution of the NO mole fractions in the burned gases as function of pressure. 
In the recent paper from de Persis et al. [172], GDFkin3.0HP_NOMecha2.0 has been tested against NO species profiles measurements performed in counterflow premixed flames stabilized up to $0.7 \mathrm{MPa}$ at three equivalence ratios. Both the GDFkin3.0HP_NOMecha2.0 and the KG-model were shown to behave similarly. Fig. 22 summarizes the experimentally measured NO peak mole fractions for a rich mixture at an equivalence ratio of $\phi=1.2$ in comparison with the kinetic model prediction. Under these conditions, where prompt-NO formation prevails, the predictions from GDFkin3.0HP_NOMecha2.0 are in better agreement with the experimental data. However, at lean conditions (see Fig. 4 in Ref. [172]), GDFkin3.0HP_NOMecha2.0 predictions overestimate the experimental results while the KG-model predictions remain within the error bars, especially at increased pressures.

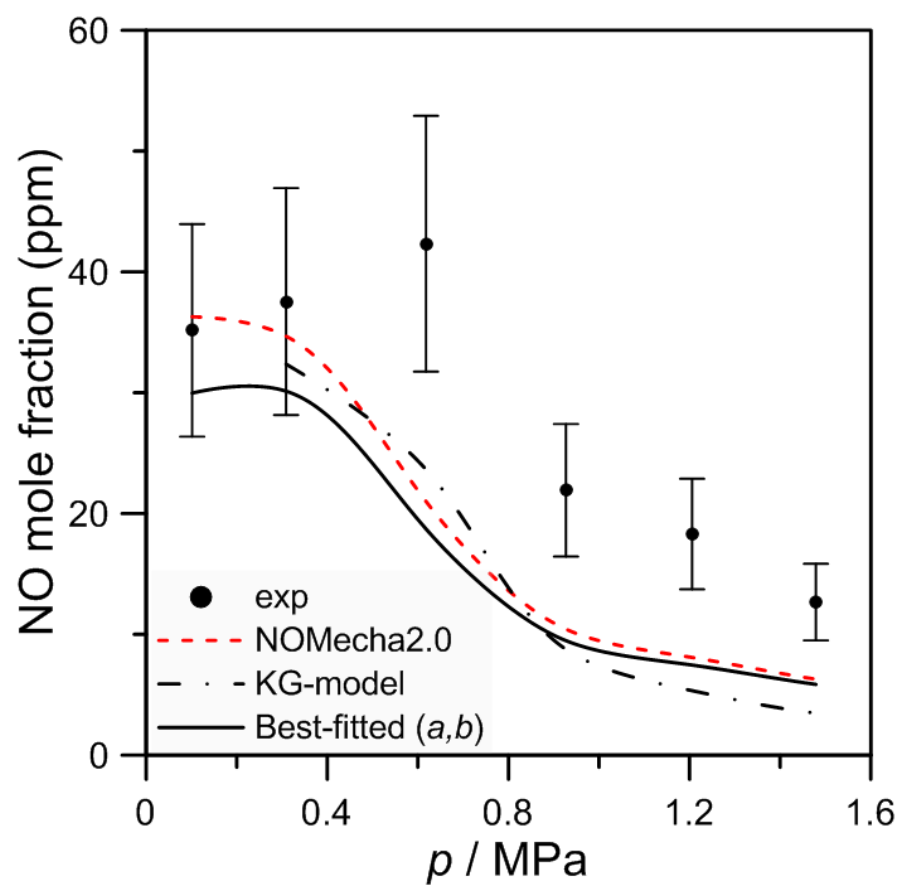

Fig. 21. Effect of pressure on the formation of $\mathrm{NO}$ in a rich premixed flame $(\phi=1.2)$. Comparison of the experimental measurements (symbols) obtained by Klassen et al. [173] with the simulated NO mole fractions using the GDFkin3.0HP_NOMecha2.0 [172] and KG-model [35]. The best-fitted line represents the simulated data obtained after the best-fit analysis performed in Section 8.2.3 with $(a=1, b=3)$. 


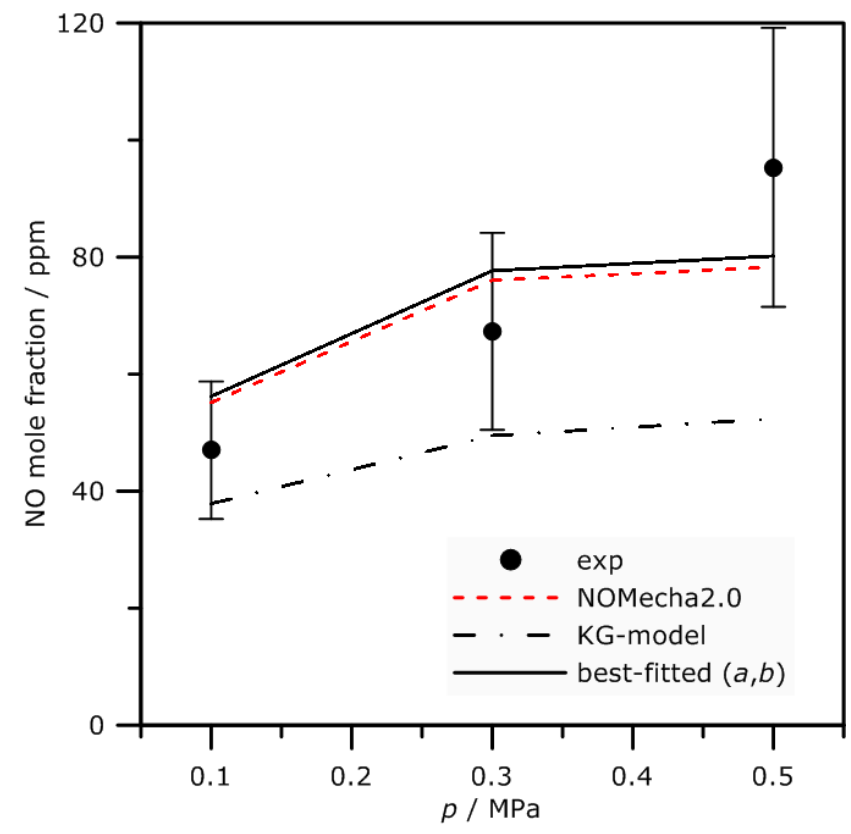

Fig. 22. Experimental peak mole fraction of NO measured in counterflow flames (symbols) [172] at $\phi=1.2$ in comparison with the simulated NO mole fractions using the GDFkin3.0HP_NOMecha2.0 [172] and KG-model [35]. The best-fitted line represents the simulated data obtained after the best-fit analysis performed in Section 8.2.3 with $(a=1, b=3)$. Adapted from de Persis et al. [172].

\subsubsection{Effective NCN consumption rates in typical flames}

In order to get further insight into the most important NCN consumption pathways in a wide range of experimental conditions, several literature flames $[52,172,173]$ have been modeled with NOMecha2.0 and the KG-model in premixed flames from low $(5.3 \mathrm{kPa})$ to high pressure $(1.5 \mathrm{MPa})$. Hereafter, the full mechanisms GDFkin ${ }^{\mathbb{}} 3.0 \_$NOMecha2.0 at low pressure or GDFkin3.0HP_NOMecha2.0 at high pressure are labelled NOMecha2.0. For estimating the relative importance of each reaction involving $\mathrm{NCN}$, the relative $\mathrm{NCN}$ consumption rate was estimated at its peak value in low-pressure flat flames (at three equivalence ratios, $\phi=0.8,1.0,1.25)$ and at $\phi=1.2$ in the pressure range $(0.1 \mathrm{MPa}<p<1.5 \mathrm{MPa}$ (flat flames) and $0.1 \mathrm{MPa}<p<0.5 \mathrm{MPa}$ (counterflow flames).

Fig. 23 summarizes the behavior of the two models with respect to the most important reactions under these conditions. Here, the relative NCN consumption rates due to each reaction were calculated based on the species mole fractions simulated with each model at the NCN peak mole fraction for each flame condition, normalized to the corresponding overall NCN consumption rate. Note that in the KGmodel, three reaction channels have been declared for the reaction $\mathrm{NCN}+\mathrm{OH}$, yielding $\mathrm{HCN}+\mathrm{NO}$, $\mathrm{NCNOH}$, and $\mathrm{NCO}+\mathrm{OH}$, but only the first one is included in NOMecha2.0 (see Section $\mathrm{C} 2$ of the Supplementary Material). Additionally, as suggested by Glarborg et al. [35], the importance of the possible reaction between $\mathrm{NCN}$ and $\mathrm{CH}_{3}$ was examined by implementing the very recent rate constant value for $\mathrm{NCN}+\mathrm{CH}_{3} \rightarrow \mathrm{CH}_{2} \mathrm{NH}+\mathrm{CN}$ from the Kiel shock tube laboratory [154]. However, as the 
product species $\mathrm{CH}_{2} \mathrm{NH}$ is neither defined in NOMecha2.0 nor the KG-model, the reaction was treated as a dead-end reaction.

From the sub-atmospheric flames to the high-pressure flames, the sensitivity analysis reveals that the most important reactions for modeling the fate of the $\mathrm{NCN}$ radical and the prompt-NO formation are $\mathrm{NCN}+\mathrm{H} \rightarrow \mathrm{CH}+\mathrm{N}_{2}$ (R-1a) and $\mathrm{NCN}+\mathrm{H} \rightarrow \mathrm{HCN}+\mathrm{N}(\mathrm{R} 2)$. Their relative importance increases with the equivalence ratio and decreases with pressure. According to NOMecha2.0, reaction (R2) prevails at all flame conditions, except in the lean low-pressure flame where the reaction (R-1a) dominates. On the contrary, according to KG-model, the latter prevails in all flames conditions. This fact clearly shows the very much different assumed branching ratios of the reactions $\mathrm{NCN}+\mathrm{H}$ in the two models, as will be further outlined in Section 8.1. Another difference between the two models is the importance of the recombination reaction channel $\mathrm{NCN}+\mathrm{H} \rightarrow \mathrm{HNCN}$ (R3). According to Klippenstein et al. [17], this pressure-dependent reaction becomes more important at pressures higher than $1 \mathrm{MPa}$ and significantly contributes to the NO decrease at very high pressures in rich conditions, in agreement with the experimental results of Ref. [173] shown in Fig. 21. The reaction (R3) remains negligible in NOMecha2.0. Finally, the fourth reaction channel of the NCN $+\mathrm{H}$ reaction, the reaction channel yielding $\mathrm{HNC}+\mathrm{N}(\mathrm{R} 4)$ remains negligible in all conditions.

In low-pressure flames, the third most important reaction is $\mathrm{NCN}+\mathrm{O} \rightarrow \mathrm{CN}+\mathrm{NO}$. Its relative importance increases from rich to lean flame conditions at low pressure. With increasing pressure at rich flame conditions, the importance of this reaction decreases, and finally falls behind the reactions NCN $+\mathrm{H}_{2}$ and $\mathrm{NCN}+\mathrm{CH}_{3}$ whose importance increases with pressure. Actually, it is interesting to see that the as yet neglected reaction $\mathrm{NCN}+\mathrm{CH}_{3}$ turns out to be quite important. However, at low pressures the NCN consumption through this pathway remains weak, but it increases with pressure at rich flame conditions. The reaction $\mathrm{NCN}+\mathrm{H}_{2} \rightarrow \mathrm{HNCN}+\mathrm{H}$ that is implemented in both models with the same rate constant is most important in high pressure flat flames. Until recently, this reaction had not been recognized and implemented into NCN submechanisms as a potentially important NCN loss pathway [80]. In an effort to clarify the importance of the $\mathrm{NCN}+\mathrm{O}$ and $\mathrm{NCN}+\mathrm{CH}_{3}$ reactions, further calculations of these flames were undertaken using NOMecha2.0. First, the rate constant value of the reaction $\mathrm{NCN}+\mathrm{O}$ taken from [111] was modified within the experimental error bars ( $\pm 40 \%)$ (see Section 6.2.4). Note that the rate constant from theoretical calculations [145] was adopted in KG-model. The modification on both the NCN rates of consumption and the species predictions was found negligible at low-pressure. However, the simulated NO mole fractions in the high-pressure stabilized flat flames increase for $p>1 \mathrm{MPa}$, reaching a better agreement with the experimental results. Second, the NCN + $\mathrm{CH}_{3} \rightarrow \mathrm{CH}_{2} \mathrm{NH}+\mathrm{CN}$ reaction was fully introduced in the model, using the $\mathrm{CH}_{2} \mathrm{NH}$ subset from Glarborg et al. [183] instead of treating this reaction as a dead-end pathway. Only a slight increase (less than 5\%) of the predicted NO mole fractions was observed for all pressures and equivalence ratios. Finally, the reaction $\mathrm{NCN}+\mathrm{OH}$ does not show up in Fig. 23. It was recently reinvestigated by 
Klippenstein et al. [17] and turns out to be much slower than many other $\mathrm{OH}$ radical reactions (see Section 6.2.6). The relative importance of this reaction somewhat increases with pressure, but remains negligible at low pressure.
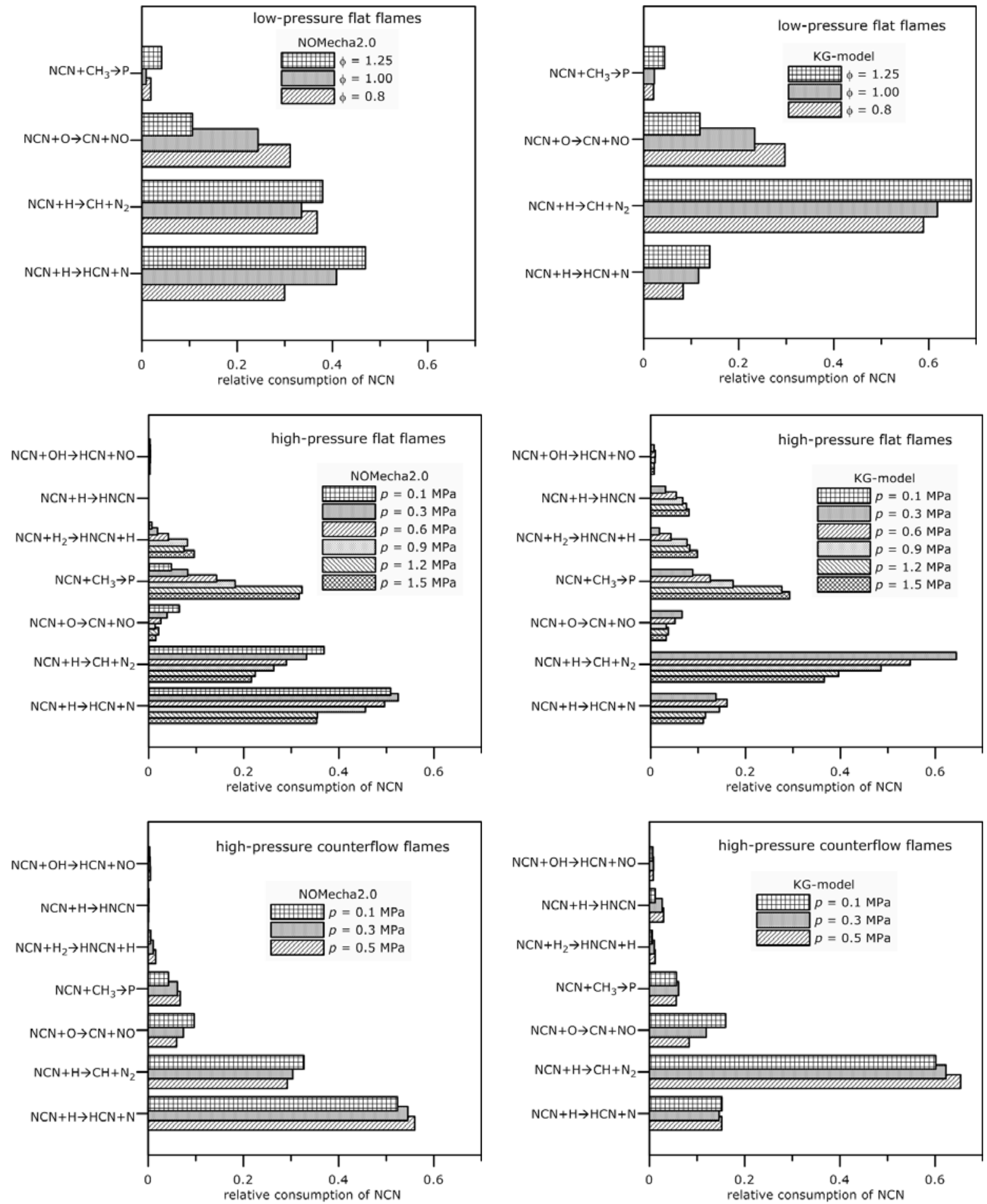

Fig. 23. Relative consumption rates of $\mathrm{NCN}$ at the NCN peak location in (top panel) low-pressure flames at three different equivalence ratios [52], in (middle panel) rich flat burner flames $(\phi=1.2)$ from 0.1 to $14.5 \mathrm{MPa}$ [173], and in (bottom panel) rich counterflow flames $(\phi=1.2)$ from 0.1 to $0.5 \mathrm{MPa}$ [172]. Simulations were performed with NOMecha2.0 (left), and with the KG-model (right). 


\section{The prompt-NO switch}

Flame modeling of prompt-NO formation is directly associated with the intermediate NCN concentration. As outlined in Section 7.2.3 and illustrated in Fig. 23, the fate of NCN is largely determined by the prompt-NO switch reaction $\mathrm{NCN}+\mathrm{H}$. Regarding the theoretical calculations performed by Teng et al. [16] and more recently by Klippenstein et al. [17], the reaction NCN $+\mathrm{H}$ proceeds via four channels yielding (R-1a) $\mathrm{CH}+\mathrm{N}_{2}$ or (R3) $\mathrm{HNCN}$ along a doublet potential energy surface, and (R2) $\mathrm{HCN}+\mathrm{N}$ or (R4) $\mathrm{HNC}+\mathrm{N}$ along a quartet potential energy surface. The analysis of the formation of nitrogen-containing species in low-pressure flames by Lamoureux et al. [52] revealed that the sensitivity of the reverse reaction (R-1a) $\mathrm{NCN}+\mathrm{H} \rightarrow \mathrm{CH}+\mathrm{N}_{2}$ is slightly lower than that of the corresponding forward reaction (R1a) $\mathrm{CH}+\mathrm{N}_{2} \rightarrow \mathrm{NCN}+\mathrm{H}$, but higher than the sensitivity of the reaction (R2) $\mathrm{NCN}+\mathrm{H} \rightarrow \mathrm{HCN}+\mathrm{N}$. The reactions centering around $\mathrm{NCN}+\mathrm{H}$, as depicted in Fig. 1, elucidated in Section 6.1., and again illustrated in a compact scheme in Fig. 24., form the complex $\mathrm{CHN}_{2}$ network that is key for prompt-NO modeling.

The most relevant model parameters are the rate constant of the forward reaction (R1a), the equilibrium constant $K_{1}$, which is required for the calculation of the rate constant of the reverse reaction (R-1a), and the rate constants and branching ratios of the three remaining channels of the $\mathrm{NCN}+\mathrm{H}$ reaction. In particular, a very sensitive balance between the dominating reactions (R-1a) and (R2) determines the setting of the prompt-NO switch, i.e. the dominating products of the reaction $\mathrm{NCN}+\mathrm{H}$.

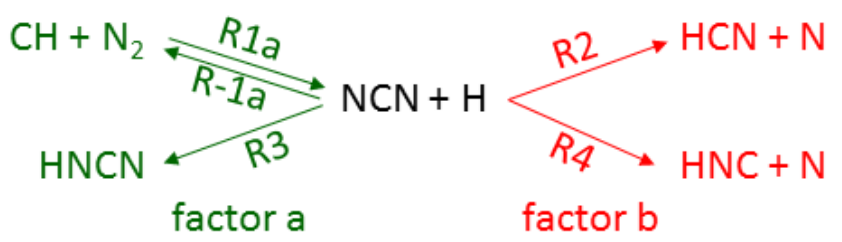

Fig. 24. Scheme depicting the most important reactions involving NCN and H. Reactions (R1a), (R-1a) and (R3) proceed on a doublet potential energy surface (green), and reactions (R2) and (R4) on a quartet potential energy surface (red) (same color code as in Fig 1). NCN modeling is crucially dependent on the ratio of doublet and quartet reaction products, factors $a$ and $b$ are introduced ad hoc in Section 8.2 to adjust the rate constants of the respective product channels.

\subsection{Relative branching fractions of the $\mathrm{NCN}+\mathrm{H}$ reactions}

The theoretically overall rate constant of the reaction $\mathrm{NCN}+\mathrm{H} \rightarrow$ products predicted by Klippenstein et al. [17] at atmospheric pressure reasonably agrees with the experimental value determined by Faßheber et al. [102], see Fig. 16. However, the predicted temperature of the shallow 
minimum of the overall rate constant, $T \approx 2200 \mathrm{~K}$, is much higher than the corresponding temperature of $T \approx \sim 1100 \mathrm{~K}$ deduced from the experimental data. According to the GDFkin ${ }^{\circledR} 3.0 \_$NOMecha2.0 model [52], which was adjusted to be consistent with the experimental data from Ref. [102], a minimum of the overall rate constant around $1000 \mathrm{~K}$ allows a satisfying prompt-NO flame modeling (see Section $7.2)$.

Fig. 25 compares relative branching fractions of the different channels of the reaction $\mathrm{NCN}+\mathrm{H}$ according to Faßheber et al. [102] (dashed curves), Lamoureux et al. [52] (dot-dashed curves), Teng et al. [16] (solid curves) and Klippenstein et al. [17] (dotted curves). As previously shown in Fig. 16, the reactions (R3) and (R4) are minor channels as compared to reactions (R-1a) and (R2), at least at low pressures and at typical flame temperatures. Note that both channels (R3) and (R4) were neglected in the analysis of Faßheber et al. [102] and the reaction channel (R4) by Lamoureux et al. [52]. In general, the reverse of the prompt-NO initiation reaction yielding $\mathrm{CH}+\mathrm{N}_{2}$ (R-1a) dominates at lower temperatures and the forward reaction toward $\mathrm{HCN}+\mathrm{N}(\mathrm{R} 2)$ at higher temperatures. The temperature at which the branching ratios of the two channels (R-1a) and (R2) intercept is called in the following the prompt-NO switch temperature, $T_{\text {switch. }}$ It is defined by the condition $k_{\text {-1a }}\left(T_{\text {switch }}\right)=k_{2}\left(T_{\text {switch }}\right)$ and represents the temperature where the branching of the reaction $\mathrm{NCN}+\mathrm{H}$ switches between reaction (R1a) (dominating at $T<T_{\text {switch }}$ ) and reaction (R2) (dominating at $T>T_{\text {switch }}$ ). Depending on the source of rate constant expressions used, it is equal to $T_{\text {switch }}=3415 \mathrm{~K}$ (Teng et al. [16]), $3235 \mathrm{~K}$ (Klippenstein et al. [17]), $1440 \mathrm{~K}$ (Faßheber et al. [102]), or $1630 \mathrm{~K}$ (Lamoureux et al. [52]).

As a result (see Fig. 23 in Section 7.2.3), the KG-model and GDFkin ${ }^{\circledR} 3.0 \_$NOMecha2.0 predict a different relative importance of reactions (R-1a) and (R2) for prompt-NO formation. At the NCN peak location in the premixed low-pressure flames of $\mathrm{CH}_{4} / \mathrm{O}_{2} / \mathrm{N}_{2}$, the temperature is around $1700 \mathrm{~K}$. This temperature is close to the value of $T_{\text {switch }}$ derived from GDFkin ${ }^{\circledR} 3.0 \_$NOMecha2.0, but much lower than the value derived from the KG-model. Accordingly, the KG-model only slightly underestimates the experimental NCN peak mole fraction, but predicts $\mathrm{NO}$ concentration that are $50 \%$ lower than the experimental value shown in Fig. 20. In contrast, with GDFkin ${ }^{\circledR} 3.0 \_$NOMecha2.0 the agreement with predicted NCN peak mole fraction becomes worse ( $45 \%$ lower than the experiment), but the simulated NO profile nicely fits the experimental data. Note that the experimental uncertainties were estimated $\pm 55 \%$ and $\pm 10 \%$ for NCN and NO, respectively. 


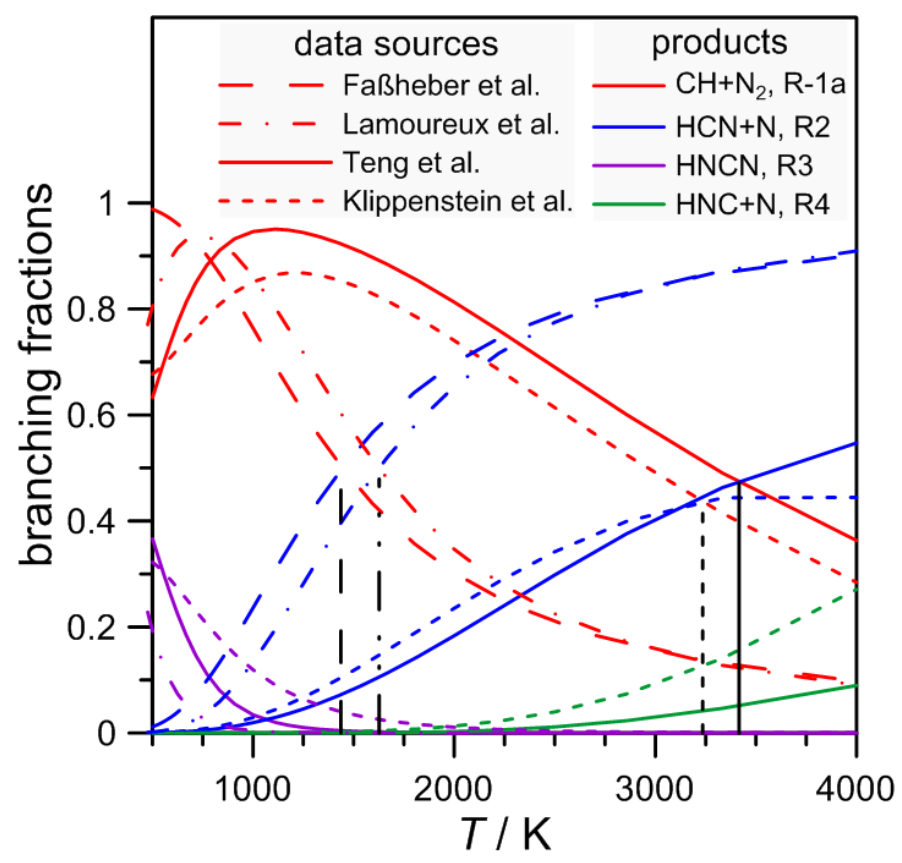

Fig. 25. Predicted branching fractions of the reaction $\mathrm{NCN}+\mathrm{H}$ at $p=0.1 \mathrm{MPa}$, according to Teng et al. [16], Faßheber et al. [102], Lamoureux et al. [52] and Klippenstein et al. [17]. The colors refer to the different reaction channels, and the line styles to the different data sources. The vertical lines indicate the prompt-NO switch temperature, $T_{\text {switch, }}$ corresponding to the crossing of the branching fractions of reaction channels (R-1a) and (R2).

\subsection{Toward a recommendation of rate constants for prompt-NO switch}

In order to evaluate the rate constant parameter set used for prompt-NO switch modeling and to find a best-fit that minimizes the deviations between the simulation and the experiments, the rate constants of the four $\mathrm{NCN}+\mathrm{H}$ reaction channels were systematically varied within a limited range. Again, the rich low-pressure $\mathrm{CH}_{4} / \mathrm{O}_{2} / \mathrm{N}_{2}$ flame whose species profiles are shown in Fig. 20 has been chosen as a validation target. The impact of these variations on the modeled peak mole fractions of the three selected species NCN, HCN, and NO was used for validation (targets 1, 2, 3, respectively). In addition, the resulting overall rate constants for the $\mathrm{NCN}+\mathrm{H}$ reaction have been compared with the experimental rate constants measured by Faßheber et al. [102] as validation target 4 . As initial values, the rate constants of the four reaction channels (R1a), (R2), (R3) and (R4) were taken from a most recent theoretical work by Klippenstein et al. [17], implemented into NOMecha2.0. For consistency with these recent dataset, also the reaction $\mathrm{CH}+\mathrm{N}_{2} \rightleftharpoons \mathrm{HNCN}$ has been incorporated. As previously discussed (Section 6.1.1), the calculation of the rate constant for the back reaction (R-1a), sensitively depends on the thermochemical parameters used. Here, one should note that some originally reported thermochemical parameters (for CH in GDFkin ${ }^{\circledR} 3.0 \_$NOMecha2.0, and for NCN in the KG-model) are inconsistent with the values reported in the majority of the other references. On the one hand, as shown in Fig. 11 in Section 3.2, the heat capacity for NCN calculated from the NASA polynomials reported in 
Ref. [17] were not in agreement with the values recalculated from the most recent available molecular data set. On the other hand, the heat capacity for $\mathrm{CH}$ used in Ref. [52] deviates from the values reported in the extended third millennium ideal gas thermochemical database [184] or in Ref. [35]. So, in the following, the thermochemical parameters for $\mathrm{CH}, \mathrm{H}, \mathrm{N}_{2}$ were taken from the KG-model, and for NCN those from Harding and Olzmann [37] were adopted (the values used are compiled in Table S5 of the Supplementary Material). As outlined in Section 3, the most recent high-level calculations of the NCN enthalpy of formation by Klippenstein et al. [17] and Harding and Olzmann [37] agree very well, therefore the thermochemical data of $\mathrm{NCN}$ were not treated as variable parameters.

The relative branching fraction between the different reaction channels of $\mathrm{NCN}+\mathrm{H}$ (see Fig. 24) was studied by applying two adjustable scaling factors, $a$ and $b$, defined as follows. The rate constants of the reactions along the doublet path (i.e., yielding $\mathrm{CH}+\mathrm{N}_{2}$ (R-1a) and $\mathrm{HNCN}$ (R3)) were multiplied by a factor $\boldsymbol{a}$ (shown in green color in Fig. 24). Because reaction (R3) is much less important than (R1a), the same scaling factor was applied to both reactions in order to restrict the number of parameters in this analysis. Note that multiplication of $k_{-1 \mathrm{a}}$ by a factor of $a$ implies multiplication of $k_{1 \mathrm{a}}$ by the same factor because the equilibrium constant $K_{\text {la }}$ was independently calculated from the thermochemical parameters of $\mathrm{CH}, \mathrm{N}_{2}, \mathrm{NCN}$, and $\mathrm{H}$. Similarly, the factor $\boldsymbol{b}$ was applied to both reactions along the quartet path (i.e., yielding $\mathrm{HCN}+\mathrm{N}(\mathrm{R} 2)$ and $\mathrm{HNC}+\mathrm{N}(\mathrm{R} 4)$, red color in Fig. 24). The influence of the relative branching fraction on the overall rate constant of the $\mathrm{NCN}+\mathrm{H}$ reaction and on the prompt-NO modelling was examined after varying the scaling factor $a$ from 0.05 to 3.0, and $b$ from 0.05 to 8.0. Note that the parameter pair $(a, b)=(1,1)$ corresponds to the original rate constant values proposed by Klippenstein et al. [17].

\subsubsection{Influence on the overall rate constant}

We assessed the influence of the channel branching on the overall rate constant of the $\mathrm{NCN}+\mathrm{H}$ reactions, $k_{\text {overall, }}$ and the switch temperature, $T_{\text {switch }}$, by varying the factors $a$ and $b$ in steps of 0.05 each.

First, the overall rate constant used in our model was compared to the experimentally determined overall rate constant, $k_{\mathrm{F}}$, from Faßheber et al. [102]. To this end, we calculated a relative deviation,

$$
\Delta k(a, b)=\int_{T_{\text {low }}}^{T_{\text {high }}}\left|k_{\text {overall }}(T ; a, b)-k_{\mathrm{F}}(T)\right| \mathrm{d} T / \int_{T_{\text {low }}}^{T_{\text {high }}} k_{\mathrm{F}}(T) \mathrm{d} T
$$

with $T_{\text {low }}=950 \mathrm{~K}$ and $T_{\text {high }}=2500 \mathrm{~K}$ (hence covering the experimental temperature range in Ref. [102]) and with $k_{\text {overall }}(T ; a, b)=a \times\left[k_{-1 \mathrm{a}}(T)+k_{3}(T)\right]+b \times\left[k_{2}(T)+k_{4}(T)\right]$. Here, $k_{\mathrm{i}}(T)$ denotes the rate constant of reaction (Ri) as given by Klippenstein et al. [17]. A contour plot of the relative deviations $\Delta k(a, b)$ is shown in Fig. 26. The deviation becomes minimal in the valley marked by the blue dashed line and it is around $27 \%$ for $(a, b)=(1,1)$. Note that an alternative scheme for weighting the differences 
based on a $\ln \left(k_{\text {overall }}\right)$ and $1 / T$ scaling (instead of $k_{\text {overall }}$ and $T$ ) yields comparable results (see Fig. S4 in the Supplementary Material).

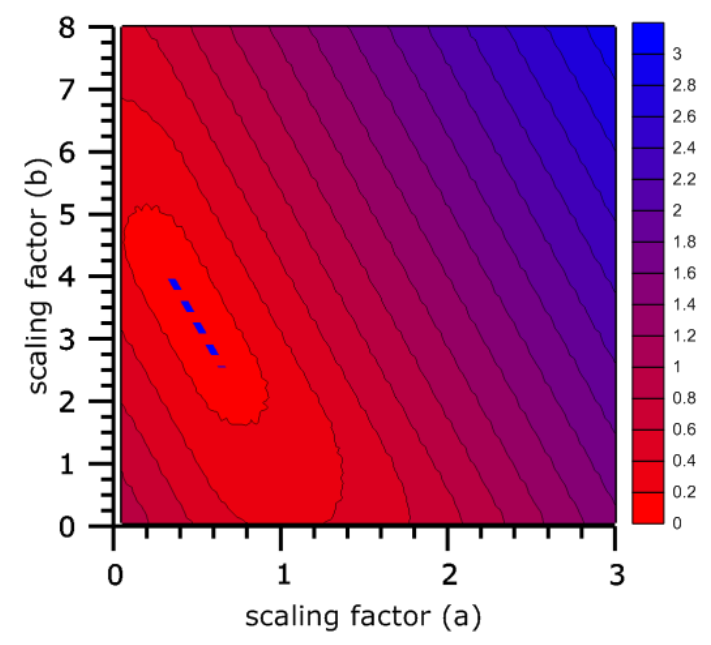

Fig. 26. Contour plot of the relative deviation $\Delta k(a, b)$; the blue dashed line represents the minimum valley of $\Delta k$, corresponding to the best agreement with the overall experimental rate constant values reported by Faßheber et al. [102] (see text).

Second, the switch temperature as function of the parameters $a$ and $b$ was calculated from the relation $a \times k_{-1 \mathrm{a}}\left(T_{\text {switch }}\right)=b \times k_{2}\left(T_{\text {switch }}\right)$. A contour plot is shown in Fig. S5 in the Supplementary Material. $T_{\text {switch }}$ decreases with an increasing ratio $b / a$ and it is equal to $3235 \mathrm{~K}$ for $(a, b)=(1,1)$.

\subsubsection{Influence on prompt-NO modeling}

The modeling of the target flame was performed with the Chemkin/Premix code. Each scaling factor $a$ and $b$ was varied with a step size of 0.2 , hence the parameter matrix represents 600 computational runs. For each sample run, the relative deviations of the peak mole fractions of the three species of interest ( $\mathrm{HCN}, \mathrm{NCN}$ and $\mathrm{NO}$ ) were calculated. The relative deviation $\Delta x$ is defined as the difference between the simulated species mole fraction value $\left(x_{\text {sim }}\right)$ and the experimental value $\left(x_{\exp }\right)$, normalized by $x_{\text {exp }}: \Delta x=\left(x_{\text {sim }}-x_{\text {exp }}\right) / x_{\text {exp }}$. The results are represented as contour plots for each species in Fig. 27. Note that the color scale is identical for all three panels. It was found that the relative deviations of the mole fraction of HCN and NO (panels b and c) follow very similar trends with varying $(a, b)$, represented by hyperbola-like contour lines. The highest deviations occur at high or low values for $a$ and $b$ (top right and bottom left corners). In order to allow for a better comparison between different species, the iso-line for an exact agreement $\left(\Delta x_{\mathrm{NO}}=0\right)$ between the simulated and experimental mole fractions of NO is plotted in all three panels of Fig. 27 as a yellow curve. It is almost identical to the iso-line for a zero deviation for $\mathrm{HCN}$, which is plotted as a green curve in panel b. In contrast, the deviation observed for the NCN peak mole fraction (panel a) exhibits a nearly linear increase from 
bottom-right to top-left. Moreover, the simulated NCN peak mole fraction does not reach the experimental value over the covered parameter range of $(a, b)$; it is always underpredicted. For a better agreement, the factor $a$ would have to be increased by an unrealistic factor of 4 . Accounting for the high experimental uncertainty in the case of $\mathrm{NCN}( \pm 50 \%)$, the black dashed line represents a relative deviation of the NCN peak mole fraction equal to $-50 \%$. Hence, agreement of the simulation with the experiment within error limit occurs on the right-hand side of this line.

Interestingly, the relative deviation of NCN behaves similarly as $T_{\text {switch }}$ (cf. Fig. S5). The higher

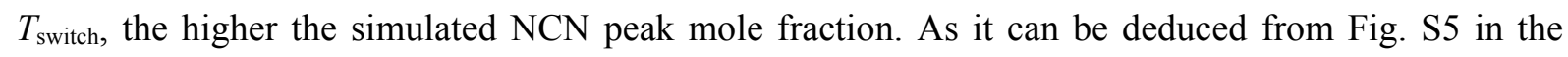
Supplementary Material, for a relative deviation of the NCN peak prediction by $-50 \%, T_{\text {switch }}$ is equal to $2000 \mathrm{~K}$. 

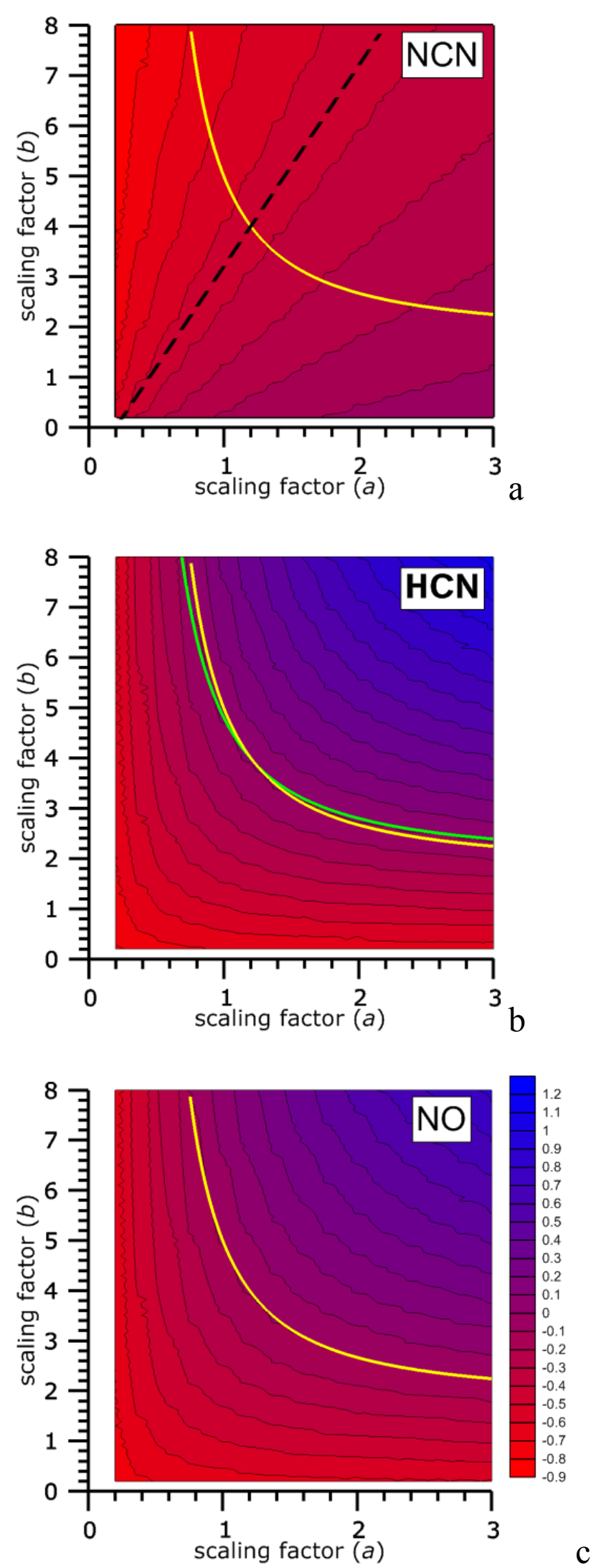

Fig. 27. Relative deviation $\Delta x$ (see text) of the experimental and the predicted peak mole fractions of NCN, HCN, and $\mathrm{NO}$ in the target low-pressure flame $\mathrm{CH}_{4} / \mathrm{O}_{2} / \mathrm{N}_{2}(\phi=1.25)$ as function of the parameter pair $(a, b)$; the color scale is identical for all panels. The yellow and green curves represent the iso-lines for zero deviation of NO and $\mathrm{HCN}$, respectively. The black dashed curve represents a NCN peak deviation of $-50 \%$. Within the examined range of $a$ and $b$, the minimal deviation of $\mathrm{NCN}$ is obtained in the bottom right corner. 


\subsubsection{Best-fit solution}

Clearly, the choice of the adjustable scaling factors $a$ and $b$ that determines the branching ratio of the prompt-NO switch reaction $\mathrm{NCN}+\mathrm{H}$ has a decisive impact on crucial quantities of the prompt-NO model. Fig. 28 collects the best-fit iso-lines (except for the NCN) for each of the target quantities described above. The yellow and green hyperbola-like curves correspond to the zero deviation iso-lines of the NO and HCN peak mole fractions, and the blue dashed line to a minimal deviation of the overall rate constant, $\Delta k \leq 0.1$. For NCN (panel a of Fig. 27), the black-dashed line indicates a $\Delta x_{\mathrm{NCN}}=-50 \%$ deviation of its peak simulation. In the selected range of the parameters, $(a \mid b) \in([0.2: 3] \mid[0.2: 8])$ where $(a \mid b)=(1 \mid 1)$ represents the rate constant values reported by Klippenstein et al. [17], the NCN deviation is minimal only for $b$ close to 0.2 and for $a$ close to 3 (see Fig. 27a). Finally, the black solid line represents a prompt-NO switch temperature of $T_{\text {switch }}=2000 \mathrm{~K}$. From the near coincidence of this line with the lower bound of the experimental uncertainty in the NCN peak mole fraction it follows that the NCN peak measurement favors a value of $T_{\text {switch }}>2000 \mathrm{~K}$. This is because higher $T_{\text {switch }}$ values result in an increase of the simulated NCN peak mole fraction, synonymous with a situation where the NCN concentration is mainly influenced by the reversible reaction pair (R1a) and (R-1a). On the contrary, if $T_{\text {switch }}$ is low, the NCN radicals are irreversibly consumed via reaction (R2) to a larger extent, which acts as a NCN sink along the $\mathrm{HCN}$-yielding Fenimore route. It turns out that $\mathrm{HCN}$ and NO kinetics are more complex, and no such direct correlation between their prediction and the NCN peak mole fraction was found. However, the $\mathrm{HCN}$ and NO predicted mole fractions tend to increase with the increase of the overall rate constant $k_{\text {overall. }}$

By combining all these different considerations, a best-fit parameter range for $a$ and $b$ can be inferred from the quantities discussed here. Keeping in mind that the corresponding experiments were often challenging, we assume that the maximum deviation in the NCN peak mole fraction should be below $\left|\Delta x_{\mathrm{NCN}}\right|=50 \%$ (in agreement with the reported experimental uncertainty). This corresponds to feasible $(a, b)$ parameter pairs within the hatched area (backward slash) of Fig. 28. For similar reasons, we allow for a maximum overall rate constant deviation of $\Delta k<0.8$, which corresponds to the hatched area (forward slash) of Fig. 28. The overlapping triangular area from these two conditions would suggest a reasonable ( $a=1.2, b=4.0)$ pair in order to be fully consistent with the HCN and NO measurements as well. However, this parameter pair is located at the top corner of the triangular double-hatched area, hence it corresponds to the maximum feasible deviation for both the total rate constant, $\Delta k=0.8$, and the NCN peak mole fraction, $\Delta x_{\mathrm{NCN}}=-50 \%$. Obviously, it is not possible to find a parameter combination that can simultaneously fulfill all four validation targets. Therefore, keeping in mind the uncertainties of all experimental quantities and also of the underlying detailed reaction mechanism and thermodynamic data used for the simulation, we consider parameter pairs $(a, b)$ located within the dashed

ellipse in Fig. 28 as acceptable solutions. Finally, tentative best-fit rate constant expressions, 
corresponding to ( $a=1.0, b=3.0)$ and a prompt-NO switch temperature of $T_{\text {switch }}=2080 \mathrm{~K}$, are adopted in Table S6 in the Supplementary Material. While $a=1$ supports the rate constant $k_{-1 \mathrm{a}}$ as given by Klippenstein et al. [17], $b=3$ favors a value of $k_{2}$ that is significantly higher than the one reported in the same publication but in much better agreement with the theoretical and experimental data provided by Teng et al. [16], Vasudevan et al. [31], and Faßheber et al. [102] (see Table 6).

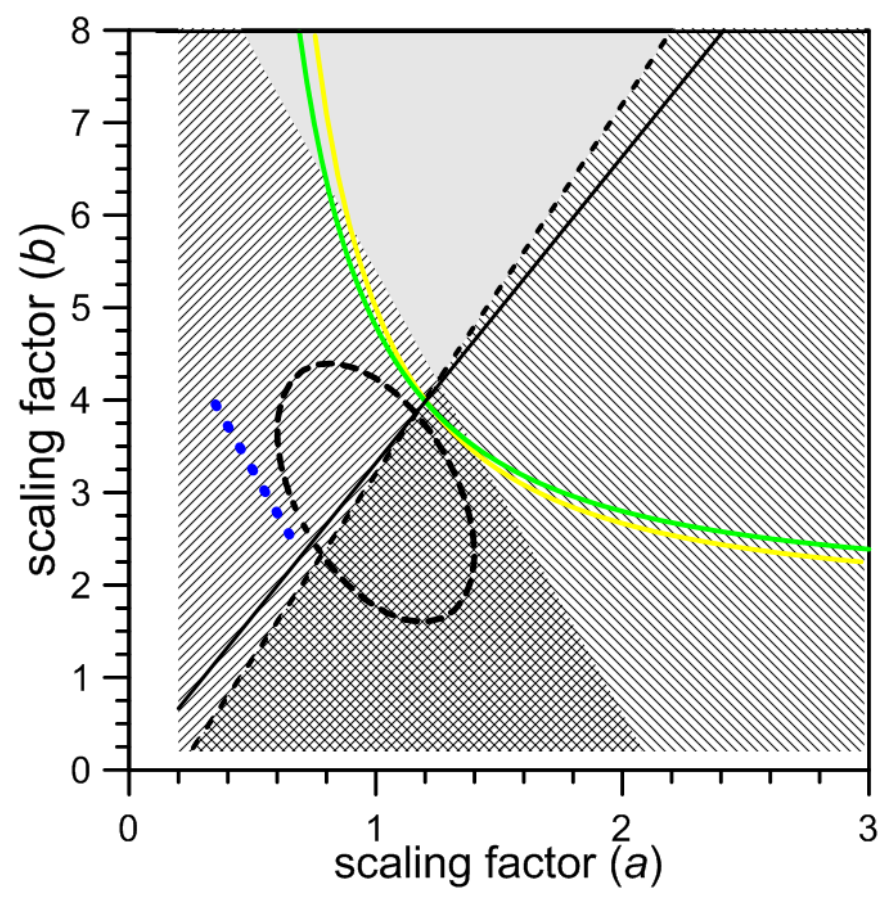

Fig. 28. Summary of the results on the relative deviations of several validation target quantities using a detailed mechanism with adjustable prompt-NO switch parameters $a$ and $b$ (see text). Blue dashed line: $\Delta k \leq 0.1$; yellow curve: $\Delta x_{\mathrm{NO}}=0$; green curve: $\Delta x_{\mathrm{HCN}}=0$; black dashed line, $\Delta x_{\mathrm{NCN}}=-50 \%$; black solid line: $T_{\text {switch }}=2000 \mathrm{~K}$ (note that $T_{\text {switch }}$ decreases with increasing ratio $b / a$ ). Backward slash hatched area: $\left|\Delta x_{\mathrm{NCN}}\right|<50 \%$; forward slash hatched area: $\Delta k<0.8$; dashed ellipse: acceptable parameter range for $a$ and $b$ to best fulfill the four validation criteria.

Note that this parameterization approach was performed for a particular target flame, i.e., a rich $\mathrm{CH}_{4} / \mathrm{O}_{2} / \mathrm{N}_{2}$ flame at $p=5.3 \mathrm{kPa}$. In this sense, the best-fit $(a, b)$ parameter pair cannot be taken as a final recommendation but needs both a more extensive validation using different flame conditions and continued experimental work, in particular for the reactions in the $\mathrm{CHN}_{2}$ system. For the sake of completeness, in Figures S6 and S7 of the Supplemental Material we have provided plots of experimentally measured and simulated best-fit species profiles using $(a, b)=(1,3)$ for several lowpressure $\mathrm{CH}_{4} / \mathrm{O}_{2} / \mathrm{N}_{2}$ and $\mathrm{C}_{2} \mathrm{H}_{2} / \mathrm{O}_{2} / \mathrm{N}_{2}$ flames [52,161], including the rich $\mathrm{CH}_{4} / \mathrm{O}_{2} / \mathrm{N}_{2}(\phi=1.25)$ flame used as the validation target. The present results show a decrease of the simulated peak mole fraction of $\mathrm{HCN}$ and NO compared to the previous calculated data in all low-pressure flames. The change of the $\mathrm{CH}$ molar heat capacity in GDFkin ${ }^{\circledR} 3.0$ model yields to a slight decrease of the simulated $\mathrm{CH}$ peak mole 
fraction in the methane flames. For the acetylene flames, modeling calculations were performed with the model proposed by Lindstedt and Skevis [171] that was slightly modified in Lamoureux et al. [161]. After combining this model (for the acetylene oxidation) and the best-fitted NOMecha2.0 model, the simulated NCN peak mole fractions agree very well with the experimental values. But the calculated NO mole fraction in the burned gases are found to be lower than the experimental ones and those obtained with the original model.

Similarly, the best-fit $(a, b)$ parameters were also introduced in the high-pressure version GDFkin3.0HP_NOMecha2.0 after substituting the rate constant values (in PLOG format) from Klippenstein et al. [17] for the reactions (R1a, R2, R3, R4, and R1c). The predicted NO mole fractions are reported (solid black curves) in Fig. 21 and Fig. 22 and compared to the experimental values obtained in flat flames and counterflow flames from 0.1 to $1.5 \mathrm{MPa}$ by Klassen et al. [173] and de Persis et al. [172], respectively. Compared to the results obtained with the original GDFkin3.0HP_NOMecha2.0, the selection of these best-fit parameters $(a=1, b=3)$ yields a very small variation of the predicted NO mole fractions at any pressure. The largest variations are observed for the flat flames stabilized at 0.1 and 0.3 MPa, but still within the experimental error bars. Consequently, although the best-fit analysis performed in this work has been restricted to a single low-pressure flame, it yields satisfying results for pressures as high as $1.5 \mathrm{MPa}$ as well.

\section{Concluding remarks}

This article aimed at an outline of the current state of knowledge on the physico-chemical properties of the cyanonitrene radical and its role in combustion chemistry. Spectroscopic, thermodynamic, and kinetic data of NCN have been reviewed in considerable detail and discussed in terms of their significance for an adequate modeling of prompt-NO formation. The most important role of the reaction sequence initialized by $\mathrm{CH}+\mathrm{N}_{2}$ leading to $\mathrm{NCN}$ was highlighted, and the dominating influences of the enthalpy of formation of $\mathrm{NCN}$ and the relative branching fractions of the $\mathrm{NCN}+\mathrm{H}$ reactions have been discussed, including the role of the NCN spin state. A simple parameterization of these branching fractions was used to model peak concentrations of several species in selected flames to compare and assess kinetic data from the literature. These modeling calculations confirmed the strong influence of the branching between the different product channels of the reaction $\mathrm{NCN}+\mathrm{H}$ and the ongoing need for more detailed kinetic data on this reaction system. In particular, the temperature- and pressure-dependent switch between the predominance of the forward channel to $\mathrm{HCN}+\mathrm{N}$ and the backward channel to $\mathrm{CH}$ $+\mathrm{N}_{2}$ needs further investigation because different models predict notably different temperatures where this switch occurs. The accurate representation of the prompt-NO switch is not only key for prompt-NO modeling along the route, $\mathrm{CH}+\mathrm{N}_{2} \rightarrow \mathrm{NCN}+\mathrm{H} \rightarrow \mathrm{HCN}+\mathrm{N}$, but also for a better assessment of the 
role of alternative bimolecular $\mathrm{NCN}$ loss reactions next to $\mathrm{NCN}+\mathrm{H}$. Here, in particular the reactions with $\mathrm{O}$ atoms, $\mathrm{CH}_{3}$ radicals and $\mathrm{H}_{2}$ turn out to open new pathways under practical combustion conditions. In this context, also the role of singlet and triplet NCN and the mechanism of ISC is of interest both for combustion modeling and from a more fundamental kinetic viewpoint.

Overall, a lot of knowledge and understanding on NCN in general and on its role in combustion chemistry has been gained over the years, in particular over the last two decades. Nonetheless, a number of important open questions remain to be solved and further experimental, theoretical, and modeling work is required to advance the understanding of NCN chemistry for prompt-NO formation in flames. Some examples necessarily reflecting the personal interests and preferences of the authors are:

- the still puzzling question of the temperature dependence of the branching ratio of the prompt$\mathrm{NO}$ switch reaction $\mathrm{NCN}+\mathrm{H}$,

- the need for detection and modeling of NCN chemistry related species profiles (including NCN itself) in atmospheric and supra-atmospheric stationary flames,

- experimental and theoretical investigations of the products and rate constants of the largely neglected $\mathrm{NCN}+\mathrm{CH}_{n}$ reactions,

- experimental verification of the NCN radical enthalpy of formation,

- a closer inspection of the so far unexplored role [185,186] of the assumed Lennard-Jones transport parameters for $\mathrm{NCN}$,

- the elucidation of the molecular details of the ${ }^{1} \mathrm{NCN} /{ }^{3} \mathrm{NCN}$ ISC process, and

- a more rigorous investigation of the potential influence of ${ }^{1} \mathrm{NCN}$ chemistry in flames and - even more important - for high-temperature kinetic experiments.

The authors hope that from this by no means exhaustive list as well as from the entire review article, it may become evident that the NCN free radical and the mechanism of prompt-NO formation are rewarding future fields of research nicely illustrating the interconnection between fundamental physical chemistry and combustion science. 


\section{Acknowledgments:}

The NCN fluorescence lifetime measurement campaign was initiated as a collaboration between Kiel and Lille within COST Action CM1404 (Smartcats: Chemistry of smart energy carriers and technology, supported by COST - European Cooperation in Science and Technology). It was conducted in the framework of a BQR International 2014 sponsorship from the University of Lille (N.L. and P.D.) and also financially supported (G.F.) by the Deutsche Forschungsgemeinschaft through research grant FR1529/4-2. We especially thank Kristian Laß and Nancy Faßheber for their active help with the setup of the laser systems.

M.O. acknowledges support of this work from the Deutsche Forschungsgemeinschaft (SFBTRR150 Turbulent, Chemically Reactive Multi Phase Flows near Walls, Projektnummer 237267381TRR150).

N.L. and P.D. would like to thank the French Ministère de l'Enseignement Supérieur et de la Recherche, the Hauts-de-France Region and the European Regional Development Funds for their financial support to this project, and this work is a contribution to the CPER research project CLIMIBIO.

We would also like to thank our group members as well as our esteemed colleagues all over the world for their tireless experimental, theoretical, and modelling work related to NCN high-temperature chemistry and for sharing and discussing their results with us. Their interest in the NCN system provided the basis and motivated us to compile this review.

\section{Supplementary Materials:}

Supplementary material associated with this article can be found in the online version at doi:10.1016/j.pecs.xxx. It contains additional information on the radiative lifetime measurements (Section 2.4.1), thermochemical data for NCN (Section 3), the NCN subsets (Sections 6 and 7) and the prompt-NO switch (Section 8).

\section{References}

[1] Jennings KR, Linnett JW. Emission spectra of flames supported by active nitrogen. Trans Faraday Soc 1960;56:1737-41. https://doi.org/10.1039/TF9605601737.

[2] Herzberg G, Travis DN. The spectrum and structure of the free NCN radical. Can J Phys 1964;42:1658-75. https://doi.org/10.1139/p64-150.

[3] Milligan DE, Jacox ME, Bass AM. Matrix Isolation Study of the Photolysis of Cyanogen Azide. The Infrared and Ultraviolet Spectra of the Free Radical NCN. J Chem Phys 1965;43:3149-60. https://doi.org/10.1063/1.1727771.

[4] Pontrelli GJ, Anastassiou AG. Formation of the NCN Radical During the Flash Photolysis of Cyanogen Azide. J Chem Phys 1965;42:3735-6. https://doi.org/10.1063/1.1695798. 
[5] Kroto HW. Singlet and Triplet States of NCN in the Flash Photolysis of Cyanogen Azide. J Chem Phys 1966;44:831-2. https://doi.org/10.1063/1.1726765.

[6] Schoen LJ. Flash Photolysis of Matrix-Isolated Cyanogen Azide in Solid Nitrogen. J Chem Phys 1966;45:2773-6. https://doi.org/10.1063/1.1728024.

[7] Moskaleva LV, Lin MC. The spin-conserved reaction $\mathrm{CH}+\mathrm{N}_{2} \rightarrow \mathrm{H}+\mathrm{NCN}$ : A major pathway to prompt no studied by quantum/statistical theory calculations and kinetic modeling of rate constant. Proc Combust Inst 2000;28:2393-401. https://doi.org/10.1016/S0082-0784(00)806529.

[8] Moskaleva LV, Xia WS, Lin MC. The $\mathrm{CH}+\mathrm{N}_{2}$ reaction over the ground electronic doublet potential energy surface: a detailed transition state search. Chem Phys Lett 2000;331:269-77. https://doi.org/10.1016/S0009-2614(00)01160-X.

[9] Fenimore CP. Formation of nitric oxide in premixed hydrocarbon flames. Symp (Int) Combust 1971;13:373-80. https://doi.org/10.1016/s0082-0784(71)80040-1.

[10] Miller JA, Bowman CT. Mechanism and modeling of nitrogen chemistry in combustion. Prog Energy Combust Sci 1989;15:287-338. https://doi.org/10.1016/0360-1285(89)90017-8.

[11] Miller JA, Pilling MJ, Troe J. Unravelling combustion mechanisms through a quantitative understanding of elementary reactions. Proc Combust Inst 2005;30:43-88. https://doi.org/10.1016/j.proci.2004.08.281.

[12] Blauwens J, Smets B, Peeters J. Mechanism of "prompt" NO formation in hydrocarbon flames. Symposium (International) on Combustion 1977;16:1055-64. https://doi.org/10.1016/S00820784(77)80395-0.

[13] Hayhurst AN, Vince IM. Nitric oxide formation from N2 in flames: The importance of "prompt" NO. Prog Energy Combust Sci 1980;6:35-51. https://doi.org/10.1016/0360-1285(80)90014-3.

[14] Berman MR, Tsuchiya T, Gregušová A, Perera SA, Bartlett RJ. HNNC Radical and Its Role in the $\mathrm{CH}+\mathrm{N}_{2}$ Reaction. J Phys Chem A 2007;111:6894-9. https://doi.org/10.1021/jp0723618.

[15] Harding LB, Klippenstein SJ, Miller JA. Kinetics of $\mathrm{CH}+\mathrm{N}_{2}$ Revisited with Multireference Methods. J Phys Chem A 2008;112:522-32. https://doi.org/10.1021/jp077526r.

[16] Teng W-S, Moskaleva LV, Chen H-L, Lin MC. Ab Initio Chemical Kinetics for H + NCN: Prediction of NCN Heat of Formation and Reaction Product Branching via Doublet and Quartet Surfaces. J Phys Chem A 2013;117:5775-84. https://doi-org/10.1021/jp402903t.

[17] Klippenstein SJ, Pfeifle M, Jasper AW, Glarborg P. Theory and modeling of relevance to promptNO formation at high pressure. Combust Flame 2018;195:3-17. https://doi.org/10.1016/j.combustflame.2018.04.029.

[18] Cui Q, Morokuma K, Bowman JM, Klippenstein SJ. The spin-forbidden reaction CH $\left({ }^{2} \Pi\right)+\mathrm{N}_{2}$ $\rightarrow \mathrm{HCN}+\mathrm{N}\left({ }^{4} \mathrm{~S}\right)$ revisited. II. Nonadiabatic transition state theory and application. J Chem Phys 1999;110:9469-82. https://doi.org/10.1063/1.478949.

[19] Cui Q, Morokuma K. The spin-forbidden reaction $\mathrm{CH}\left({ }^{2} \Pi+\right) \mathrm{N}_{2} \rightarrow \mathrm{HCN}+\mathrm{N}\left({ }^{4} \mathrm{~S}\right)$ revisited I. Ab initio study of the potential energy surfaces. Theor Chem Acc 1999;102:127-33. https://doi.org/10.1007/s002140050482.

[20] Turro NJ, Ramamurthy V, Scaiano JC. Modern molecular photochemistry of organic molecules. Sausalito CA: University Science Books; 2010.

[21] Berman MR, Lin MC. Kinetics and mechanism of the methylidyne + molecular nitrogen reaction. Temperature- and pressure-dependence studies and transition-state-theory analysis. J Phys Chem 1983;87:3933-42. https://doi.org/10.1021/j100243a028.

[22] Fulle D, Hippler H. The high-pressure range of the reaction of $\mathrm{CH}\left({ }^{2} \Pi\right)$ with $\mathrm{N}_{2}$. J Chem Phys 1996;105:5423-30. https://doi.org/10.1063/1.472383.

[23] Moskaleva LV, Lin MC. The $\mathrm{CH}+\mathrm{N}_{2}$ Association Reaction at Low Temperatures: Ab Initio MO/VRRKM-Theory Analysis of Temperature and Pressure Effects. Z Phys Chem 2001;215:1043-54. https://doi.org/10.1524/zpch.2001.215.8.1043 |.

[24] Rodgers AS, Smith GP. Pressure and temperature dependence of the reactions of $\mathrm{CH}$ with $\mathrm{N}_{2}$. Chem Phys Lett 1996;253:313-21. https://doi.org/10.1016/0009-2614(96)00204-7.

[25] Miller JA, Walch SP. Prompt NO: Theoretical prediction of the high-temperature rate coefficient for $\mathrm{CH}+\mathrm{N}_{2} \rightarrow \mathrm{HCN}+\mathrm{N}$. Int J Chem Kinet 1997;29:253-9. https://doi.org/10.1002/(sici)10974601(1997)29:4<253::aid-kin3>3.0.co;2-t. 
[26] Riad Manaa M, Yarkony DR. On the mechanism of the reaction $\mathrm{CH}\left(\mathrm{X}^{2} \Pi\right)+\mathrm{N}_{2}\left(\mathrm{X}{ }^{1} \Sigma_{\mathrm{g}}^{+}\right) \rightarrow$ $\mathrm{HCN}\left(\mathrm{X}^{1} \Sigma^{+}\right)+\mathrm{N}\left({ }^{4} \mathrm{~S}\right)$. I. A theoretical treatment of the electronic structure aspects of the intersystem crossing. J Chem Phys 1991;95:1808-16. https://doi.org/10.1063/1.461028.

[27] Riad Manaa M, Yarkony DR. The mechanism of the reaction $\mathrm{CH}\left(\mathrm{X}^{2} \Pi\right)+\mathrm{N}_{2}\left(\mathrm{X}^{1} \Sigma_{\mathrm{g}}^{+}\right) \rightarrow \mathrm{HCN}$ $\left(\mathrm{X}^{1} \Sigma^{+}\right)+\mathrm{N}\left({ }^{4} \mathrm{~S}\right)$. II. The intermediate complex region. Chem Phys Lett 1992;188:352-8. https://doi.org/10.1016/0009-2614(92)90036-M.

[28] Martin JML, Taylor PR. The mechanism of the reaction $\mathrm{CH}+\mathrm{N}_{2} \rightarrow \mathrm{HCN}+\mathrm{N}$. Chem Phys Lett 1993;209:143-50. https://doi.org/10.1016/0009-2614(93)87215-O.

[29] Walch SP. Characterization of the minimum energy path for $\mathrm{CH}\left(\mathrm{X}^{2} \Pi\right)+\mathrm{N}_{2}\left(\mathrm{X}^{1} \Sigma_{\mathrm{g}}^{+}\right) \rightarrow \mathrm{HCN}$ $\left(\mathrm{X}^{1} \Sigma^{+}\right)+\mathrm{N}\left({ }^{4} \mathrm{~S}\right)$. Chem Phys Lett 1993;208:214-8. https://doi.org/10.1016/00092614(93)89064-O.

[30] Takayanagi T. A CASPT2 study of the doublet potential energy surface for the $\mathrm{CH}\left(\mathrm{X}^{2} \Pi\right)+$ $\mathrm{N}_{2}\left(\mathrm{X}^{1} \Sigma_{\mathrm{g}}^{+}\right)$reaction. Chem Phys Lett 2003;368:393-8. https://doi.org/10.1016/S00092614(02)01887-0.

[31] Vasudevan V, Hanson RK, Bowman CT, Golden DM, Davidson DF. Shock Tube Study of the Reaction of CH with $\mathrm{N}_{2}$ : Overall Rate and Branching Ratio. J Phys Chem A 2007;111:1181830. https://doi.org/10.1021/jp075638c.

[32] Dean AJ, Hanson RK, Bowman CT. High temperature shock tube study of reactions of $\mathrm{CH}$ and C-atoms with $\mathrm{N}_{2}$. Symp (Int) Combust 1991;23:259-65. https://doi.org/10.1016/s00820784(06)80268-7.

[33] El Bakali A, Pillier L, Desgroux P, Lefort B, Gasnot L, Pauwels JF, et al. NO prediction in natural gas flames using GDF-Kin ${ }^{\circledR} 3.0$ mechanism NCN and $\mathrm{HCN}$ contribution to prompt-NO formation. Fuel 2006;85:896-909. https://doi.org/10.1016/j.fuel.2005.10.012.

[34] Turányi T, Tomlin AS. Analysis of kinetic reaction mechanisms. Heidelberg: Springer, Berlin, Heidelberg; 2014.

[35] Glarborg P, Miller JA, Ruscic B, Klippenstein SJ. Modeling nitrogen chemistry in combustion. Prog Energy Combust Sci 2018;67:31-68. https://doi.org/10.1016/j.pecs.2018.01.002.

[36] Goos E, Sickfeld C, Mauß F, Seidel L, Ruscic B, Burcat A, et al. Prompt NO formation in flames: The influence of NCN thermochemistry. Proc Combust Inst 2013;34:657-66. https://doi.org/10.1016/j.proci.2012.06.128.

[37] Harding ME, Olzmann M. High-accuracy extrapolated ab initio thermochemistry of the NCN radical. Chem Phys Lett 2018;706:613-6. https://doi.org/10.1016/j.cplett.2018.06.047.

[38] Smith GP. Evidence of NCN as a flame intermediate for prompt NO. Chem Phys Lett 2003;367:541-8. https://doi.org/10.1016/S0009-2614(02)01739-6.

[39] Smith GP, Copeland RA, Crosley DR. Electronic quenching, fluorescence lifetime, and spectroscopy of the A ${ }^{3} \Pi_{u}$ state of NCN. J Chem Phys 1989;91:1987-93. https://doiorg/10.1063/1.457057.

[40] Berg PA, Hill DA, Noble AR, Smith GP, Jeffries JB, Crosley DR. Absolute CH concentration measurements in low-pressure methane flames: comparisons with model results. Combust Flame 2000;121:223-35. https://doi.org/10.1016/S0010-2180(99)00130-3.

[41] Sutton JA, Williams BA, Fleming JW. Laser-induced fluorescence measurements of NCN in low-pressure $\mathrm{CH}_{4} / \mathrm{O}_{2} / \mathrm{N}_{2}$ flames and its role in prompt $\mathrm{NO}$ formation. Combust Flame 2008;153:465-78. https://doi.org/10.1016/j.combustflame.2007.09.008.

[42] Lamoureux N, Mercier X, Western C, Pauwels JF, Desgroux P. NCN quantitative measurement in a laminar low pressure flame. Proc Combust Inst 2009;32:937-44. https://doi.org/10.1016/j.proci.2008.06.043.

[43] Klein-Douwel RJH, Dam NJ, ter Meulen JJ. Laser-induced fluorescence of NCN in low and atmospheric pressure flames. Opt Lett 2008;33:2620-2. https://doi.org/10.1364/OL.33.002620.

[44] Lamoureux N, Desgroux P, El Bakali A, Pauwels JF. Experimental and numerical study of the role of NCN in prompt-NO formation in low-pressure $\mathrm{CH}_{4}-\mathrm{O}_{2}-\mathrm{N}_{2}$ and $\mathrm{C}_{2} \mathrm{H}_{2}-\mathrm{O}_{2}-\mathrm{N}_{2}$ flames. Combust Flame 2010;157:1929-41. https://doi.org/10.1016/j.combustflame.2010.03.013.

[45] Sun ZW, Dam NJ, Li ZS, Aldén M. NCN detection in atmospheric flames. Combust Flame 2010;157:834-6. https://doi.org/10.1016/j.combustflame.2009.12.012. 
[46] Sutton JA, Williams BA, Fleming JW. Investigation of NCN and prompt-NO formation in lowpressure C1-C4 alkane flames. Combust Flame 2012;159:562-76. https://doi.org/10.1016/j.combustflame.2011.08.023.

[47] Glarborg P, Alzueta MU, Dam-Johansen K, Miller JA. Kinetic Modeling of Hydrocarbon/Nitric Oxide Interactions in a Flow Reactor. Combust Flame 1998;115:1-27. https://doi.org/10.1016/S0010-2180(97)00359-3.

[48] Dagaut P, Glarborg P, Alzueta MU. The oxidation of hydrogen cyanide and related chemistry. Prog Energy Combust Sci 2008;34:1-46. https://doi.org/10.1016/j.pecs.2007.02.004.

[49] Konnov AA. Implementation of the NCN pathway of prompt-NO formation in the detailed reaction mechanism. Combust Flame 2009;156:2093-105. https://doi.org/10.1016/j.combustflame.2009.03.016.

[50] Sepman AV, Mokhov AV, Levinsky HB. The effects of the hydrogen addition on the HCN profiles in fuel-rich-premixed, burner-stabilized C1-C3 alkane flames. Int J Hydrogen Energy 2011;36:13831-7. https://doi.org/10.1016/j.ijhydene.2011.07.090.

[51] Zsély IG, Zádor J, Turányi T. Uncertainty analysis of NO production during methane combustion. Int J Chem Kinet 2008;40:754-68. https://doi.org/10.1002/kin.20373.

[52] Lamoureux N, El Merhubi H, Pillier L, de Persis S, Desgroux P. Modeling of NO formation in low pressure premixed flames. Combust Flame 2016;163:557-75. http://dx.doi.org/10.1016/j.combustflame.2015.11.007.

[53] Moskaleva LV, Lin MC. Computational Study on the Energetics of NCN Isomers and the Kinetics of the $\mathrm{C}+\mathrm{N}_{2} \rightarrow \mathrm{N}+\mathrm{CN}$ Reaction. J Phys Chem A 2001;105:4156-63. https://doi.org/10.1021/jp0044328.

[54] Sanders WA, Lin CY, Lin MC. On The Importance Of The Reaction $\mathrm{CH}_{2}+\mathrm{N}_{2} \rightarrow \mathrm{HCN}+\mathrm{NH}$ As A Precursor For Prompt No Formation. Combust Sci Technol 1987;51:103-8. https://doiorg/10.1080/00102208708960318.

[55] Williams BA, Sutton JA, Fleming JW. The role of methylene in prompt NO formation. Proceedings of the Combustion Institute 2009;32 I:343-50. https://doi.org/10.1016/j.proci.2008.06.175.

[56] Xu S, Lin MC. Ab Initio Chemical Kinetics for Singlet $\mathrm{CH}_{2}$ Reaction with $\mathrm{N}_{2}$ and the Related Decomposition of Diazomethane. J Phys Chem A 2010;114:5195-204. https://doi.org/10.1021/jp911048p.

[57] Kolbanovskii YA, Borisov YA. Quantum chemical calculations on the mechanism of the reaction between dicarbene $\mathrm{C}_{2}\left(\mathrm{X}^{1} \Sigma^{+} \mathrm{g}\right)$ and molecular nitrogen. Russ J Phys Chem B 2015;9:29-35. https://doi.org/10.1134/S1990793115010169.

[58] Sommer T, Kruse T, Roth P, Hippler H. Perturbation study on the reaction of $\mathrm{C}_{2}$ with $\mathrm{N}_{2}$ in hightemperature $\mathrm{C}_{60} / \mathrm{Ar}+\mathrm{N}_{2}$ mixtures. J Phys Chem A 1997;101:3720-5. https://doiorg/10.1021/jp962779y.

[59] Williams BA, Fleming JW. Experimental and modeling study of NO formation in 10 torr methane and propane flames: Evidence for additional prompt-NO precursors. Proc Combust Inst 2007;31:1109-17. https://doi.org/10.1016/j.proci.2006.07.246.

[60] Zhu RS, Xu SC, Lin MC. Ab initio chemical kinetics for the reactions of $\mathrm{N}_{2}$ with singlet and triplet $\mathrm{C}_{2} \mathrm{O}$ radicals. Chem Phys Lett 2010;488:121-5. https://doi.org/10.1016/j.cplett.2010.02.003.

[61] An F, Han S, Hu X, Xie D, Guo H. First-principles dynamics of collisional intersystem crossing: resonance enhanced quenching of $C\left({ }^{1} D\right)$ by $N_{2}$. Phys Chem Chem Phys 2019;21:8645-53. https://doi.org/10.1039/C8CP07171C.

[62] Hickson KM, Loison J-C, Lique F, Kłos J. An Experimental and Theoretical Investigation of the $\mathrm{C}\left({ }^{1} \mathrm{D}\right)+\mathrm{N}_{2} \rightarrow \mathrm{C}\left({ }^{3} \mathrm{P}\right)+\mathrm{N}_{2}$ Quenching Reaction at Low Temperature. J Phys Chem A 2016;120:2504-13. https://doi.org/10.1021/acs.jpca.6b00480.

[63] Kroto HW. The ${ }^{1} \Pi_{\mathrm{u}} \leftarrow{ }^{1} \Delta_{\mathrm{g}}$ electronic spectrum of NCN. Can J Phys 1967;45:1439-50. https://doi.org/10.1139/p67-108.

[64] Taylor TR, Bise RT, Asmis KR, Neumark DM. The singlet-triplet splittings of NCN. Chem Phys Lett 1999;301:413-6. https://doi.org/10.1016/S0009-2614(99)00053-6. 
[65] Perić M, Krmar M, Radić-Perić J, Hanrath M. Ab Initio Investigation of the $\ldots \pi_{\mathrm{g}}^{2}\left(\mathrm{X}^{3} \Sigma_{\mathrm{g}}^{-}, 1^{1} \Delta_{\mathrm{g}}\right.$, $1^{1} \Sigma_{\mathrm{g}}^{+}$) Electronic States of NCN. Study of the Renner-Teller Effect in the $1^{1} \Delta_{\mathrm{g}}$ State. J Mol Spectrosc 2000;204:226-34. https://doi.org/10.1006/jmsp.2000.8222.

[66] Bise RT, Choi H, Neumark DM. Photodissociation dynamics of the singlet and triplet states of the NCN radical. J Chem Phys 1999;111:4923-32. https://doi.org/10.1063/1.479751.

[67] Kroto HW, Morgan TF, Sheena HH. Flash photolysis of cyanogen azide, $\mathrm{NCN}_{3}$. Trans Faraday Soc 1970;66:2237-43. https://doi.org/10.1039/TF9706602237.

[68] Lamoureux N, Western CM, Mercier X, Desgroux P. Reinvestigation of the spectroscopy of the $\tilde{\mathrm{A}}^{3} \prod_{\mathrm{u}}-\tilde{\mathrm{X}}^{3} \Sigma_{\mathrm{g}}{ }^{-}$transition of the NCN radical at high temperature: Application to quantitative NCN measurement in flames. Combust Flame 2013;160:755-65. https://doi.org/10.1016/j.combustflame.2012.12.009.

[69] Beaton SA, Ito Y, Brown JM. Laser excitation spectroscopy of the $\tilde{\mathrm{A}}^{3} \Pi_{\mathrm{u}}-\tilde{\mathrm{X}}^{3} \Sigma_{\mathrm{g}}^{-}$transition of the NCN radical: 1. The (0-0) band. J Mol Spectrosc 1996;178:99-107. https://doi.org/10.1006/jmsp.1996.0162.

[70] Beaton SA, Brown JM. Laser Excitation Spectroscopy of the $\tilde{A}^{3} \Pi_{u^{-}} \tilde{X}^{3} \Sigma_{g}^{-}$Transition of the NCN Radical 2. The $\mathrm{N}_{2}$ Hot Band. J Mol Spectrosc 1997;183:347-59. https://doi.org/10.1006/jmsp.1997.7286.

[71] Perić M, Krmar M, Radić-Perić J, Stevanović L. Ab Initio Investigation of the Renner-Teller Effect in the $\mathrm{A}^{3} \Pi_{\mathrm{u}}$ Electronic State of NCN. J Mol Spectrosc 2001;208:271-80. https://doi.org/10.1006/jmsp.2001.8384.

[72] Dammeier J, Friedrichs G. Thermal Decomposition of $\mathrm{NCN}_{3}$ as a High-Temperature NCN Radical Source: Singlet-Triplet Relaxation and Absorption Cross Section of $\mathrm{NCN}\left({ }^{3} \Sigma\right)$. J Phys Chem A 2010;114:12963-71. https://doi.org/10.1021/jp1043046.

[73] Hetzler J, Olzmann M. Collisional Relaxation of NCN: An Experimental Study with LaserInduced Fluorescence. Z Phys Chem 2015;229:1503-19. https://doi.org/10.1515/zpch-20150673.

[74] Western CM. PGOPHER: A program for simulating rotational, vibrational and electronic spectra. J Quant Spectrose Radiat Transfer 2017; 186:221-42. http://dx.doi.org/10.1016/j.jqsrt.2016.04.010.

[75] Jeffries JB, Copeland RA, Smith GP, Crosley DR. Multiple species laser-induced fluorescence in flames. Symp (Int) Combust 1988;21:1709-18. https://doi.org/10.1016/S00820784(88)80404-1.

[76] McNaughton D, Metha GF, Tay R. Generation of transient species by laser induced pyrolysis. The high resolution fourier transform infrared spectrum of NCN. Chem Phys 1995;198:107-17. https://doi.org/10.1016/0301-0104(95)00120-D.

[77] Hensel KD, Brown JM. The $v_{3}$ Band of the NCN Radical, Studied by LMR. J Mol Spectrosc 1996;180:170-4. https://doi.org/10.1006/jmsp.1996.0238.

[78] Wienkoop M, Urban W, Brown JM. The NCN $21^{1} 3{ }_{1}{ }^{0}$ Hot Band Measured by Infrared Laser Magnetic Resonance Spectroscopy. J Mol Spectrosc 1997;185:185-6. https://doi.org/10.1006/jmsp.1997.7346.

[79] Whiting EE, Schadee A, Tatum JB, Hougen JT, Nicholls RW. Recommended conventions for defining transition moments and intensity factors in diatomic molecular spectra. J Mol Spectrosc 1980;80:249-56. https://doi.org/10.1016/0022-2852(80)90137-X.

[80] Faßheber N, Bornhorst L, Hesse S, Sakai Y, Friedrichs G. Kinetic modelling and shock tube study on the reactions ${ }^{3} \mathrm{NCN}+\mathrm{H}_{2}$ and ${ }^{1} \mathrm{NCN}+\mathrm{H}_{2}$. J Phys Chem A 2020;124:4632-45. https://doi.org/10.1021/acs.jpca.0c02631.

[81] Friedrichs G, Colberg M, Fikri M, Huang Z, Neumann J, Temps F. Validation of the Extended Simultaneous Kinetics and Ringdown Model by Measurements of the Reaction $\mathrm{NH}_{2}+\mathrm{NO}$. J Phys Chem A 2005;109:4785-95. https://doi.org/10.1021/jp0508599.

[82] Dammeier J, Oden B, Friedrichs G. A consistent model for the thermal decomposition of $\mathrm{NCN}_{3}$ and the singlet- triplet relaxation of NCN. Int J Chem Kinet 2013;45:30-40. https://doi.org/10.1002/kin.20739.

[83] Mayer JE, Goeppert Mayer M. Statistical mechanics. New-York: John Wiley \& Sons; 1940.

[84] Pitzer K. Thermodynamics. 3rd ed. New York: McGraw-Hill; 1995. 
[85] Helgaker T, Klopper W, Tew DP. Quantitative quantum chemistry. Mol Phys 2008;106:210743. https://doi.org/10.1080/00268970802258591.

[86] Breidung J, Thiel W. Prediction of vibrational spectra from ab initio theory. In: Quack M, Merkt F, editors. Handbook of High-resolution spectroscopy, vol. 1: Fundamentals and theory, Chichester: Wiley; 2011, p. 389-403.

[87] Herzberg G. Molecular spectra and molecular structure. vol. 2: Infrared and Raman spectra of polyatomic molecules. New-York: Van Nostrand; 1945.

[88] Milligan DE, Jacox ME. Matrix-Isolation Study of the Photolysis of Cyanogen Azide. II. The Symmetric Stretching Fundamental of the Free Radical NCN. J Chem Phys 1966;45:1387-91. https://doi.org/10.1063/1.1727771.

[89] Martin JML, Taylor PR, François JP, Gijbels R. Ab initio study of the spectroscopy and thermochemistry of the $\mathrm{C}_{2} \mathrm{~N}$ and $\mathrm{CN}_{2}$ molecules. Chem Phys Lett 1994;226:475-83. https://doi.org/10.1016/0009-2614(94)00758-6.

[90] Rajendra P, Chandra P. Ground and valence excited states of $\mathrm{C}_{2} \mathrm{~N}$ and $\mathrm{CN}_{2}$ transients: $\mathrm{Ab}$ initio geometries, electronic structures, and molecular properties. J Chem Phys 2001;114:1589-600. https://doi.org/10.1063/1.1333701.

[91] Canneaux S, Wallet A, Ribaucour M, Louis F. A theoretical study of the NCN $\left({ }^{3} \Sigma^{-}\right)$biradical thermochemical properties: Implications for combustion chemistry. Comput Theor Chem 2011;967:67-74. https://doi.org/10.1016/j.comptc.2011.03.044.

[92] Klippenstein SJ, Harding LB, Ruscic B. Ab Initio Computations and Active Thermochemical Tables Hand in Hand: Heats of Formation of Core Combustion Species. J Phys Chem A 2017;121:6580-602. https://doi.org/10.1021/acs.jpca.7b05945.

[93] Jacox ME. Vibrational and Electronic Energy Levels of Polyatomic Transient Molecules. Supplement B. J Phys Chem Ref Data 2003;32:1-441. https://doi.org/10.1063/1.1497629.

[94] McBride BJ, Gordon S. Computer program for calculating and fitting thermodynamic functions. NASA; 1992.

[95] McBride BJ, Gordon S, Reno MA. Coefficients for calculating thermodynamic and transport properties of individual species. NASA; 1993.

[96] Burcat A, Gardiner Jr. WC. Ideal gas thermochemical data for combustion and air pollution use. In: Gardiner Jr. WC, editor. Gas-phase combustion chemistry, New-York: Springer; 2000, p. 489-538.

[97] Goos E, Burcat A. Overview of thermochemistry and its application to reaction kinetics. In: DaCosta H, Fan M, editors. Rate constant calculation for thermal reactions: Methods and Applications, New-York: Wiley; 2012, p. 3-32.

[98] McBride BJ, Zehe MJ, Gordon S. NASA Glenn coefficients for calculating thermodynamic properties of individual species. NASA; 2002.

[99] Benson S. Thermochemical kinetics. 2nd ed. New-York: Wiley; 1976.

[100] Chase Jr MW. NIST-JANAF Thermochemical Tables. vol. 9. 4th ed. Journal of Physical and Chemical Reference Data Monograph; 1998.

[101] Clifford EP, Wenthold PG, Lineberger WC, Petersson GA, Ellison GB. Photoelectron Spectroscopy of the NCN- and HNCN- Ions. J Phys Chem A 1997;101:4338-45. https://doi.org/10.1021/jp964067d.

[102] Faßheber N, Dammeier J, Friedrichs G. Direct measurements of the total rate constant of the reaction $\mathrm{NCN}+\mathrm{H}$ and implications for the product branching ratio and the enthalpy of formation of NCN. Phys Chem Chem Phys 2014;16:11647-57. https://doi.org/10.1039/c4cp01107d.

[103] Bugler J, Somers KP, Simmie JM, Güthe F, Curran HJ. Modeling Nitrogen Species as Pollutants: Thermochemical Influences. J Phys Chem A 2016;120:7192-7. https://doi.org/10.1021/acs.jpca.6b05723.

[104] Ruscic B. Active Thermochemical Tables (ATcT) of the thermochemical network 2013. https://atct.anl.gov/Thermochemical Data (accessed March 14, 2019).

[105] Ruscic B. Active Thermochemical Tables (ATcT) of the thermochemical network 2015. https://atct.anl.gov/Thermochemical Data (accessed March 14, 2019).

[106] Ruscic B. Active Thermochemical Tables (ATcT) of the thermochemical network 2020. https://atct.anl.gov/Thermochemical Data (accessed May 27, 2021). 
[107] Baren RE, Hershberger JF. Kinetics of the NCN Radical. J Phys Chem A 2002;106:11093-7. https://doi.org/10.1021/jp0208844.

[108] Yang T-J, Wang NS, Lee LC, Xu ZF, Lin MC. Kinetics and Mechanism of the $\mathrm{NCN}+\mathrm{NO}_{2}$ Reaction Studied by Experiment and Theory. J Phys Chem A 2008;112:10185-92. https://doiorg/10.1021/jp805311u.

[109] Welz O, Olzmann M. Kinetics of the NCN + NO Reaction over a Broad Temperature and Pressure Range. J Phys Chem A 2012;116:7293-301. https://doi.org/10.1021/jp303069g.

[110] Huang C-L, Tseng SY, Wang TY, Wang NS, Xu ZF, Lin MC. Reaction mechanism and kinetics of the NCN + NO reaction: Comparison of theory and experiment. J Chem Phys 2005;122:184321. https://doi.org/10.1063/1.1896361.

[111] Dammeier J, Faßheber N, Friedrichs G. Direct measurements of the high temperature rate constants of the reactions $\mathrm{NCN}+\mathrm{O}, \mathrm{NCN}+\mathrm{NCN}$, and NCN + M. Phys Chem Chem Phys 2012;14:1030-7. https://doi.org/10.1039/C1CP22123J.

[112] Dammeier J, Friedrichs G. Direct Measurements of the Rate Constants of the Reactions NCN + $\mathrm{NO}$ and $\mathrm{NCN}+\mathrm{NO}_{2}$ Behind Shock Waves. J Phys Chem A 2011;115:14382-90. https://doi.org/10.1021/jp208715c.

[113] Faßheber N, Lamoureux N, Friedrichs G. The rate constant of the reaction $\mathrm{NCN}+\mathrm{H}_{2}$ and its role in NCN and NO modeling in low pressure $\mathrm{CH}_{4} / \mathrm{O}_{2} / \mathrm{N}_{2}$-flames. Phys Chem Chem Phys 2015;17:15876-86. https://doi.org/10.1039/c5cp01414j.

[114] Faßheber N, Friedrichs G. Shock Tube Measurements of the Rate Constant of the Reaction NCN $+\mathrm{O}_{2}$. Int J Chem Kinet 2015;47:586-95. https://doi.org/10.1002/kin.20932.

[115] Busch A, Olzmann M. Shock-Tube study of the thermal decomposition of NCN. Proceedings of the European combustion meeting, Vienna, Austria: 2009, p. P810138.

[116] Busch A, González-García N, Lendvay G, Olzmann M. Thermal Decomposition of NCN: ShockTube Study, Quantum Chemical Calculations, and Master-Equation Modeling. J Phys Chem A 2015;119:7838-46. https://doi.org/10.1021/acs.jpca.5b01347.

[117] Marsh FD, Hermes ME. Azodicarbonitrile. J Am Chem Soc 1965;87:1819-20. https://doi.org/10.1021/ja01086a054.

[118] Marsh FD. Cyanogen Azide. J Org Chem 1972;37:2966-9. https://doi.org/10.1021/jo00984a012.

[119] Bock H, Dammel R. The Pyrolysis of Azides in the Gas Phase. Angew Chem Int Ed 1987;26:504-26. https://doi.org/10.1002/anie.198705041.

[120] Bak B, Bang O, Nicolaisen F, Rump O. Assignment of vibrational frequencies in the infrared and Raman spectra of cyanogen azide. Spectrochim Acta, Part A 1971;27:1865-71. https://doi.org/10.1016/0584-8539(71)80239-8.

[121] Busch A. Stoßwellenuntersuchungen zum Zerfall stickstoffhaltiger Verbindungen mit spektroskopischen Methoden. Doctoral dissertation. KIT Scientific Publishing, Karlsruhe, 2010.

[122] Benard DJ, Linnen C, Harker A, Michels HH, Addison JB, Ondercin R. Dissociation of Cyanogen Azide: An Alternative Route to Synthesis of Carbon Nitride. J Phys Chem A 1998;102:6010-9. https://doi.org/10.1021/jp9818479.

[123] Ci C-G, Duan X-M, Liu J-Y, Sun C-C. Photodissociation Mechanism of Cyanogen Azide. Acta Phys Chim Sin 2010;26:2787-92.

[124] Pfeifle M, Georgievskii Y, Jasper AW, Klippenstein SJ. Theoretical investigation of intersystem crossing in the cyanonitrene molecule, ${ }^{1} \mathrm{NCN} \rightarrow{ }^{3} \mathrm{NCN}$. J Chem Phys 2017;147:084310. https://doi.org/10.1063/1.4999788.

[125] Okabe H, Mele A. Photodissociation of $\mathrm{NCN}_{3}$ in the Vacuum-Ultraviolet Production of $\mathrm{CN} \mathrm{B}^{2} \Sigma$ and NCN A ${ }^{3} \Pi$. J Chem Phys 1969;51:2100-6. https://doi.org/10.1063/1.1672305.

[126] Baumgärtel S, Gericke KH, Comes FJ. Characterisation of $\mathrm{CN}$ in the photodissociation of cyanogen azide at $193 \mathrm{~nm}$. Ber Bunsen Ges Phys Chem 1994;98:1009-14. https://doi.org/10.1002/bbpc.19940980804.

[127] Friedrichs G, Wagner HG. Investigation of the Thermal Decomposition of Ketene and of the Reaction $\mathrm{CH}_{2}+\mathrm{H}_{2} \rightleftarrows \mathrm{CH}_{3}+\mathrm{H}$. $\mathrm{Z}$ Phys Chem 2001;215:1601. https://doi.org/10.1524/zpch.2001.215.12.1601.

[128] Jones JM, Moss R. Singlet Carbenes. In: Moss R, Platz M, Jones Jr M, editors. Reactive Intermediate Chemistry, Wiley-Interscience; 2004, p. 273-328. 
[129] Tomioka H. Triplet Carbenes. In: Moss R, Platz M, Jones Jr M, editors. Reactive Intermediate Chemistry, Wiley-Interscience; 2004, p. 375-461.

[130] Platz M. Nitrenes. In: Moss R, Platz M, Jones Jr M, editors. Reactive intermediate chemistry, Wiley-Interscience; 2004, p. 501-59.

[131] Mackie JC, Bacskay GB. Quantum chemical study of the mechanism of reaction between NH (X ${ }^{3} \Sigma^{-}$) and $\mathrm{H}_{2}, \mathrm{H}_{2} \mathrm{O}$, and $\mathrm{CO}_{2}$ under combustion conditions. J Phys Chem A 2005;109:11967-74. https://doi.org/10.1021/jp0544585.

[132] Anastassiou AG, Shepelavy JN. Electronic multiplicity of photolytically generated cyanonitrene. J Am Chem Soc 1968;90:492-3. https://doi.org/10.1021/ja01004a050.

[133] Becker KH, Engelhardt B, Geiger H, Kurtenbach R, Schrey G, Wiesen P. Temperature dependence of the $\mathrm{CH}+\mathrm{N}_{2}$ reaction at low total pressure. Chem Phys Lett 1992;195:322-8. https://doi.org/10.1016/0009-2614(92)85610-M.

[134] Lindackers D, Burmeister M, Roth P. Pertubation studies of high temperature $\mathrm{C}$ and $\mathrm{CH}$ reactions with $\mathrm{N}_{2}$ and NO. Proc Combust Inst 1991;23:251-7. https://doi.org/10.1016/s00820784(06)80267-5.

[135] Lamoureux N, Desgroux P, El Bakali A, Pauwels JF. Corrigendum to "Experimental and numerical study of the role of NCN in the prompt-NO formation in low pressure $\mathrm{CH}_{4} / \mathrm{O}_{2} / \mathrm{N}_{2}$ and $\mathrm{C}_{2} \mathrm{H}_{2} / \mathrm{O}_{2} / \mathrm{N}_{2}$ flames" [Combust. Flame 157 (2010) 1929-1941]. Combust Flame 2013;160:7456. https://doi.org/10.1016/j.combustflame.2012.11.013.

[136] Lamoureux N, El-Bakali A, Gasnot L, Pauwels JF, Desgroux P. Prompt-NO formation in methane/oxygen/nitrogen flames seeded with oxygenated volatile organic compounds: Methyl ethyl ketone or ethyl acetate. Combust Flame 2008;153:186-201. https://doi.org/10.1016/j.combustflame.2007.07.011.

[137] Rolland S, Simmie JM. Http://Www.Nuigalway.Ie/Combustionchemistrycentre/Softwaredownloads/Chemrevv130/: 2004.

[138] Varga T, Zsély IG, Turányi T, Bentz T, Olzmann M. Kinetic analysis of ethyl iodide pyrolysis based on shock tube measurements. Int J Chem Kinet 2014;46:295-304. https://doi.org/10.1002/kin.20829.

[139] Harvey JN. Understanding the kinetics of spin-forbidden chemical reactions. Phys Chem Chem Phys 2007;9:331-43. https://doi.org/10.1039/B614390C.

[140] Chen H-T, Ho J-J. Theoretical Investigation of the Mechanisms of Reaction of NCN with NO and NS. J Phys Chem A 2005;109:2564-71. https://doi.org/10.1021/jp045060w.

[141] Wei Z-G, Li Q-S, Zhang S-W, Sun Y-B, Sun C-C. Quantum mechanical studies on the potential energy surface of the reactions ${ }^{3} \mathrm{NCN} /{ }^{3} \mathrm{CNN}+\mathrm{NO},{ }^{1} \mathrm{NNO}+\mathrm{CN}$ and $2 \mathrm{~N}_{3}+\mathrm{CO}$. J Mol Struct THEOCHEM 2005;722:139-46. https://doi.org/10.1016/j.theochem.2004.11.055.

[142] Kappler C. Untersuchungen komplexbildender bimolekularer Reaktionen in der Gasphase mit laserspektroskopischen Methoden und statistischer Reaktionstheorie. doctoral dissertation. KIT Scientific Publishing, Karlsruhe, 2010.

[143] Husain D, Kirsch LJ. Reactions of atomic carbon $C\left(2^{3} \mathrm{P}_{\mathrm{J}}\right)$ by kinetic absorption spectroscopy in the vacuum ultra-violet. Trans Faraday Soc 1971;67:2025-35. https://doi.org/10.1039/TF9716702025.

[144] Gannon KL, Blitz MA, Pilling MJ, Seakins PW, Klippenstein SJ, Harding LB. Kinetics and product branching ratios of the reaction of ${ }^{1} \mathrm{CH}_{2}$ with $\mathrm{H}_{2}$ and $\mathrm{D}_{2}$. J Phys Chem A 2008;112:957583. https://doi.org/10.1021/jp803038s.

[145] Zhu RS, Lin MC. Ab Initio Study on the Oxidation of NCN by O $\left({ }^{3} \mathrm{P}\right)$ : Prediction of the Total Rate Constant and Product Branching Ratios. J Phys Chem A 2007;111:6766-71. https://doi.org/10.1021/jp068991b.

[146] Röhrig M, Petersen EL, Davidson DF, Hanson RK. Pressure dependence of the thermal decomposition of $\mathrm{N}_{2} \mathrm{O}$. Int J Chem Kinet 1996;28:599-608. https://doi.org/10.1002/(SICI)10974601(1996)28:8<599::AID-KIN5>3.0.CO;2-Q.

[147] Zhu RS, Lin MC. Ab initio study of the oxidation of NCN by $\mathrm{O}_{2}$. Int J Chem Kinet 2005;37:5938. https://doi.org/10.1002/kin.20066.

[148] Borden WT, Hoffmann R, Stuyver T, Chen B. Dioxygen: What Makes This Triplet Diradical Kinetically Persistent? J Am Chem Soc 2017;139:9010-8. https://doi.org/10.1021/jacs.7b04232. 
[149] Zhu RS, Nguyen HMT, Lin MC. Ab Initio Study on the Oxidation of NCN by OH: Prediction of the Individual and Total Rate Constants. J Phys Chem A 2009;113:298-304. https://doi.org/10.1021/jp805821x.

[150] Sutton JA, Fleming JW. Towards accurate kinetic modeling of prompt NO formation in hydrocarbon flames via the NCN pathway. Combust Flame 2008;154:630-6. https://doi.org/10.1016/j.combustflame.2008.05.009.

[151] Zhu RS, Lin MC. Ab initio study on the oxidation of NCN by O and HO radicals: Prediction of the total rate constant and products branching ratios, Gaithersburg, MD, USA: 2005, p. abstract M1.

[152] Moskaleva LV. Computational studies of combustion systems pertinent to NOx and incipient soot formation. Doctoral dissertation. Emory University, Atlanta, 2001.

[153] Moskaleva LV, Lin MC. Computational study of the $\mathrm{H} / \mathrm{CH}_{3}$ reactions with NCN. Fall Technical Meeting, The Eastern States Section, Combustion Institute, 2001.

[154] Hesse S, Lamoureux N, Friedrichs G. Importance of the reactions $\mathrm{NCN}+\mathrm{OH}$ and $\mathrm{NCN}+\mathrm{CH}_{3}$ for NOx formation: shock tube measurements and flame modeling. 37th Symposium international on Combustion, Symposium International on Combustion, Dublin: 2018, p. 2 P006.

[155] Pang GA, Hanson RK, Golden DM, Bowman CT. High-temperature measurements of the rate constants for reactions of $\mathrm{OH}$ with a series of large normal alkanes: n-pentane, $\mathrm{n}$-heptane, and nnonane. Z Phys Chem 2011;225:1157-78. https://doi.org/10.1524/zpch.2011.0156.

[156] $\mathrm{He} \mathrm{Y}, \mathrm{Wu} \mathrm{CH}, \mathrm{Lin} \mathrm{MC}$, Melius CF. The Reaction CN + NO at High Temperature in Shock Wave. 19th int. Symp. Shock Waves, Marseille, France: 1995.

[157] McIlroy A, Jeffries JB. Cavity Ringdown Spectroscopy for Concentration Measurements in Flames. In: Kohse-Höinghaus K, Jeffries JB, editors. Applied Combustion Diagnostics, New York: Taylor \& Francis; 2002, p. 98-127.

[158] Friedrichs G. Sensitive absorption methods for quantitative gas phase kinetic measurements. Part 2: Cavity ringdown spectroscopy. Z Phys Chem 2008;222:31-61. https://doi.org/10.1524/zpch.2008.222.1.31.

[159] Mercier X, Desgroux P. Cavity Ring-Down Spectroscopy for combustion studies. In: Berden G, Engeln R, editors. Cavity Ring-Down Spectroscopy: Techniques and Applications, WileyBlackwell; 2009, p. 273-311.

[160] Cheskis S, Derzy I, Lozovsky VA, Kachanov A, Romanini D. Cavity ring-down spectroscopy of $\mathrm{OH}$ radicals in low pressure flame. Appl Phys B 1998;66:377-81. https://doi.org/10.1007/s003400050404.

[161] Lamoureux N, Gasnot L, Desgroux P. Quantitative NH measurements by using laser-based diagnostics in low-pressure flames. Proceedings of the Combustion Institute 2019;37:1313-20. https://doi.org/10.1016/j.proci.2018.09.007.

[162] Lamoureux N, El Merhubi H, Gasnot L, Schoemaecker C, Desgroux P. Measurements and modelling of $\mathrm{HCN}$ and $\mathrm{CN}$ species profiles in laminar $\mathrm{CH}_{4} / \mathrm{O}_{2} / \mathrm{N}_{2}$ low pressure flames using LIF/CRDS techniques. Proc Combust Inst 2015;35:745-52. http://dx.doi.org/10.1016/j.proci.2014.05.126.

[163] Lamoureux N, Mercier X, Pauwels J-F, Desgroux P. NCO Quantitative Measurement in Premixed Low Pressure Flames by Combining LIF and CRDS Techniques. J Phys Chem A 2011;115:5346-53. https://doi.org/10.1021/jp201453k.

[164] Mercier X, Jamette P, Pauwels JF, Desgroux P. Absolute CH concentration measurements by cavity ring-down spectroscopy in an atmospheric diffusion flame. Chem Phys Lett 1999;305:334-42. https://doi.org/10.1016/S0009-2614(99)00416-9.

[165] Klein-Douwel RJH, Konnov AA, Dam NJ, ter Meulen JJ. NCN concentration and interfering absorption by $\mathrm{CH}_{2} \mathrm{O}, \mathrm{NH}$ and $\mathrm{OH}$ in low pressure methane/air flames with and without $\mathrm{N}_{2} \mathrm{O}$. Combust Flame 2011;158:2090-104. https://doi.org/10.1016/j.combustflame.2011.04.009.

[166] Berg PA, Smith GP, Jeffries JB, Crosley DR. Nitric Oxide Formation and Reburn in LowPressure Methane Flames. Symp (Int) Combust 1998;27:1377-84. https://doi.org/10.1016/S0082-0784(98)80543-2.

[167] Gasnot L, Desgroux P, Pauwels JF, Sochet LR. Detailed analysis of low-pressure premixed flames of $\mathrm{CH}_{4}+\mathrm{O}_{2}+\mathrm{N}_{2}$ : A study of prompt-NO. Combust Flame 1999;117:291-306. https://doi.org/10.1016/s0010-2180(98)00078-9. 
[168] Pillier L, El Bakali A, Mercier X, Rida A, Pauwels JF, Desgroux P. Influence of C2 and C3 compounds of natural gas on NO formation: An experimental study based on LIF/CRDS coupling. Proc Combust Inst 2005;30:1183-91. https://doi.org/10.1016/j.proci.2004.08.057.

[169] Smith GP, Golden DM, Frenklach M, Moriarty NW, Eiteneer B, Goldenberg M, et al. GRIMech3.0. GRI-Mech n.d. http://combustion.berkeley.edu/gri-mech/version30/text30.html.

[170] Wang H, You X, Joshi AV, Davis SG, Laskin A, Egolfopoulos F, et al. USC Mech Version II. High-Temperature Combustion Reaction Model of H2/CO/C1-C4 Compounds 2007. http://ignis.usc.edu/Mechanisms/USC-Mech\%20II/USC_Mech\%20II.htm.

[171] Lindstedt RP, Skevis G. Chemistry of Acetylene Flames. Combust Sci Technol 1997;125:73137.

[172] de Persis S, Pillier L, Idir M, Molet J, Lamoureux N, Desgroux P. NO formation in high pressure premixed flames: Experimental results and validation of a new revised reaction mechanism. Fuel 2020;260:116331. https://doi.org/10.1016/j.fuel.2019.116331.

[173] Klassen MS, Thomsen DD, Reisel JR, Laurendeau NM. Laser-Induced Fluorescence Measurements of Nitric Oxide Formation in High-Pressure Premixed Methane Flames. Combust Sci Technol 1995;110-111:229-47. https://doi.org/10.1080/00102209508951925.

[174] Naik SV, Laurendeau NM. Measurements of Absolute CH Concentrations by Cavity Ring-Down Spectroscopy and Linear Laser-Induced Fluorescence in Laminar, Counterflow Partially Premixed and Nonpremixed Flames at Atmospheric Pressure. Appl Opt 2004;43:5116-25. https://doi.org/10.1364/AO.43.005116.

[175] Naik SV, Laurendeau NM. LIF measurements and chemical kinetic analysis of nitric oxide formation in high-pressure counterflow partially premixed and nonpremixed flames. Combust Sci Technol 2004;176:1809-53. https://doi.org/10.1080/00102200490504472.

[176] Dyakov IV, Ruyck JD, Konnov AA. Probe sampling measurements and modeling of nitric oxide formation in ethane+air flames. Fuel 2007;86:98-105. https://doi.org/10.1016/j.fuel.2006.06.003.

[177] Konnov AA, Dyakov IV, Ruyck JD. The effects of composition on the burning velocity and NO formation in premixed flames of $\mathrm{C}_{2} \mathrm{H}_{4}+\mathrm{O}_{2}+\mathrm{N}_{2}$. Exp Therm Fluid Sci 2008;32:1412-20. https://doi.org/10.1016/j.expthermflusci.2007.11.017.

[178] Brackmann C, Methling T, Lavadera ML, Capriolo G, Konnov AA. Experimental and modeling study of nitric oxide formation in premixed methanol + air flames. Combust Flame 2020;213:322-30. https://doi.org/10.1016/j.combustflame.2019.11.043.

[179] Versailles P, Watson GMG, Lipardi ACA, Bergthorson JM. Quantitative CH measurements in atmospheric-pressure, premixed flames of C1-C4 alkanes. Combust Flame 2016;165:109-24. http://dx.doi.org/10.1016/j.combustflame.2015.11.001.

[180] Watson GMG, Versailles P, Bergthorson JM. NO formation in rich premixed flames of C1-C4 alkanes and alcohols. Proc Combust Inst 2017;36:627-35. https://doi.org/10.1016/j.proci.2016.06.108.

[181] Watson GMG, Versailles P, Bergthorson JM. NO formation in premixed flames of C1-C3 alkanes and alcohols. Combust Flame 2016;169:242-60. https://doi.org/10.1016/j.combustflame.2016.04.015.

[182] Versailles P, Durocher A, Bourque G, Bergthorson JM. Nitric oxide formation in lean, methaneair stagnation flames at supra-atmospheric pressures. Proc Combust Inst 2019;37:711-8. https://doi.org/10.1016/j.proci.2018.05.060.

[183] Glarborg P, Andreasen CS, Hashemi H, Qian R, Marshall P. Oxidation of methylamine. International Journal of Chemical Kinetics 2020;52:893-906. https://doi.org/10.1002/kin.21408.

[184] Goos E, Burcat A, Ruscic B. Extended Third Millennium Ideal Gas and Condensed Phase Thermochemical Database for Combustion with updates from Active Thermochemical Tables 2010. https://www.dlr.de/vt/en/desktopdefault.aspx/tabid-7603/12862_read-32379/ (accessed October 2, 2012).

[185] Langer R, Lotz J, Cai L, vom Lehn F, Leppkes K, Naumann U, et al. Adjoint sensitivity analysis of kinetic, thermochemical, and transport data of nitrogen and ammonia chemistry. Proceedings of the Combustion Institute 2021;38:777-85. https://doi.org/10.1016/j.proci.2020.07.020.

[186] Miller JA, Sivaramakrishnan R, Tao Y, Goldsmith CF, Burke MP, Jasper AW, et al. Combustion chemistry in the twenty-first century: Developing theory-informed chemical kinetics models. 
Progress in Energy and Combustion Science 2021;83:100886. https://doi.org/10.1016/j.pecs.2020.100886. 